University of Tennessee Health Science Center

UTHSC Digital Commons

\title{
Development of Multiple Microemulsion Eye Drops for Sustained Release of New Glaucoma Drug
}

\author{
Doaa Nabih Ahmed Maria \\ University of Tennessee Health Science Center
}

Follow this and additional works at: https://dc.uthsc.edu/dissertations

Part of the Medicinal and Pharmaceutical Chemistry Commons, and the Pharmaceutics and Drug Design Commons

\section{Recommended Citation}

Maria, Doaa Nabih Ahmed (http://orcid.org/0000-0003-2845-5237), "Development of Multiple Microemulsion Eye Drops for Sustained Release of New Glaucoma Drug" (2018). Theses and Dissertations (ETD). Paper 466. http://dx.doi.org/10.21007/etd.cghs.2018.0473. 


\title{
Development of Multiple Microemulsion Eye Drops for Sustained Release of New Glaucoma Drug
}

\author{
Abstract \\ Persistent or repeated elevation of intraocular pressure (IOP) is a primary risk factor of visual field loss in \\ glaucoma, therefore IOP reduction is the first-line therapeutic option in the disease management. \\ Unfortunately, the current therapies are associated with a lot of deficiencies including several daily \\ dosing, reduced efficacy and systemic side effects all of which resulted in poor patient compliance. \\ Previously we have identified Calcium voltage-gated channel auxiliary subunit Alpha2delta 1 gene \\ (Cacna2d1) as a novel modulator of IOP and confirmed that pregabalin targeted CACNA2D1 in eye \\ tissues (ciliary body and trabecular meshwork) to lower IOP in a dose-dependent manner. The research \\ presented in this dissertation aimed to develop a once-daily ocular pregabalin-loaded multiple water-in-oil- \\ in-water microemulsion eye drops. Several in vitro and in vivo evaluations were used to characterize the \\ prepared ophthalmic formulations. Also stability study at $5^{\circ} \mathrm{C}, 25^{\circ} \mathrm{C}, 30^{\circ} \mathrm{C}$ and $40^{\circ} \mathrm{C}$ was conducted for \\ four months. All the formulations components were carefully selected to be highly biocompatible that \\ provided a highly transparent eye drops with a miniscule droplet size ( \\ Document Type \\ Dissertation \\ Degree Name \\ Doctor of Philosophy (PhD) \\ Program \\ Pharmaceutical Sciences \\ Research Advisor \\ Jablonski , Monica M., PhD \\ Keywords \\ Dutch belted rabbits, Glaucoma, Intraocular pressure, Microemulsion, Stability, Transcorneal permeability \\ Subject Categories \\ Medicinal and Pharmaceutical Chemistry | Medicine and Health Sciences | Pharmaceutics and Drug \\ Design | Pharmacy and Pharmaceutical Sciences
}




\title{
Development of Multiple Microemulsion Eye Drops for Sustained Release of New Glaucoma Drug
}

\author{
A Dissertation \\ Presented for \\ The Graduate Studies Council \\ The University of Tennessee \\ Health Science Center
}

\author{
In Partial Fulfillment \\ Of the Requirements for the Degree \\ Doctor of Philosophy \\ From The University of Tennessee
}

By

Doaa Nabih Ahmed Maria

December 2018 
Copyright (C) 2018 by Doaa Nabih Ahmed Maria. All rights reserved. 


\section{DEDICATION}

To my mother and to the spirit of my father. 


\section{ACKNOWLEDGEMENTS}

First of all I would like to thank ALLAH my god most gracious, most merciful for helping me to go through this work and for letting it come to a successful end.

I would like to express my heartily thanks and appreciation to my advisor, professor Dr. Monica M. Jablonski for her guidance, sincere help and infinite support all the times. I will forever be thankful to her for giving me this wonderful opportunity to be her mentee. Each time we meet she boost my confidence so much and in that is something I really was in need specially before any presentation that I have. I would like to thank her for being much more than an advisor as our meetings never feel like formal advisor-student meetings. Prof. Monica shown me by her example what a good scientist and person should be. Honestly, she is such a wonderful advisor who care about her students not only concerning research work but much more about how they feel.

I would like to thank the members of my committee. Dr. Hassan Almoazen, Dr. Soumyajit Majumdar, Dr. Nawajes Mandal and Dr. Bernd Meibohm for their highly important suggestions, discussions and constructive criticism. I appreciate each of your insight and encouragement throughout this process.

I would like to express my deepest thanks to my beloved parents who sacrificed to give me every opportunity and whose love, support and guidance are with me in whatever I pursue. My deepest thanks to my husband and my three lovely wonderful hero children, Amr, Nouran and Ahmed, who provide unending inspiration and were patient with a mother who was busy all the time.

I want to thank the current and past members of Dr. Jablonski lab; Mohamed Moustafa, XiangDi Wang, Raven Davis, Sumana Chintalapudi, Ellen Garner and Mallika Palamoor who kindly supported me. 


\begin{abstract}
Persistent or repeated elevation of intraocular pressure (IOP) is a primary risk factor of visual field loss in glaucoma, therefore IOP reduction is the first-line therapeutic option in the disease management. Unfortunately, the current therapies are associated with a lot of deficiencies including several daily dosing, reduced efficacy and systemic side effects all of which resulted in poor patient compliance. Previously we have identified Calcium voltage-gated channel auxiliary subunit Alpha2delta 1 gene (Cacna2d1) as a novel modulator of IOP and confirmed that pregabalin targeted CACNA2D1 in eye tissues (ciliary body and trabecular meshwork) to lower IOP in a dose-dependent manner. The research presented in this dissertation aimed to develop a once-daily ocular pregabalin-loaded multiple water-in-oil-in-water microemulsion eye drops. Several in vitro and in vivo evaluations were used to characterize the prepared ophthalmic formulations. Also stability study at $5^{\circ} \mathrm{C}, 25^{\circ} \mathrm{C}, 30^{\circ} \mathrm{C}$ and $40^{\circ} \mathrm{C}$ was conducted for four months. All the formulations components were carefully selected to be highly biocompatible that provided a highly transparent eye drops with a miniscule droplet size $(<20 \mathrm{~nm})$ accompanied with a good shelf life stability. The special engineering of the microemulsion eye drops succeeded to provide a continuous pregabalin release for up to $24 \mathrm{~h}$. Slit-lamp biomicroscopic exams and the cell toxicity study demonstrated that the developed ocular formulations were non-toxic. The in vivo study using Dutch belted rabbits showed that the microemulsion formulation markedly enhanced the efficacy and prolonged the duration of pregabalin IOP-lowering effect. Interestingly, a single drop of pregabalin microemulsion induced $42.3 \pm 2.6 \%$ IOP reduction that returned to baseline at $32.7 \pm 1.3 \mathrm{~h}$ from application (AUC $=169.9 \pm 13.4$ mmHg.h). In the absence of the microemulsion system, the same drug produced only $29.4 \pm 1.4 \%$ IOP reduction that returned to baseline at $9.3 \pm 0.7 \mathrm{~h}(\mathrm{AUC}=39 \pm 4 \mathrm{mmHg} . \mathrm{h}$ ). Finally, the developed multiple microemulsion is a promising carrier that sustained the release and prolonged the duration of action of pregabalin and could be a carrier for any other water-soluble drug.
\end{abstract}




\section{TABLE OF CONTENTS}

CHAPTER 1. INTRODUCTION ....................................................................................1

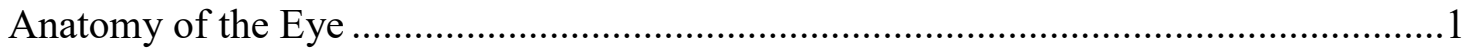

Eyebrows and eyelids ................................................................................ 1

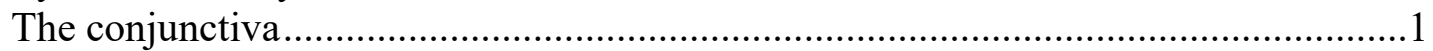

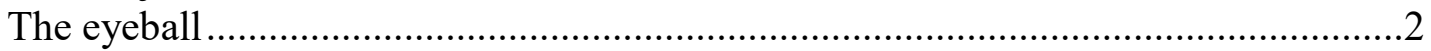

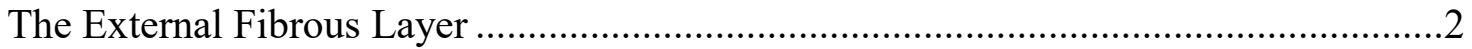

The sclera (the white of the eye) ......................................................................

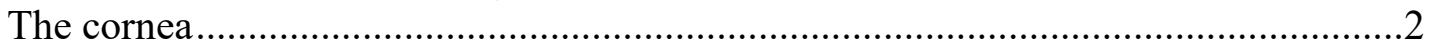

The Intermediate Vascular/Muscular Layer ..............................................................

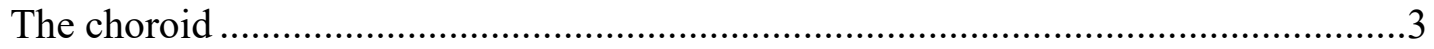

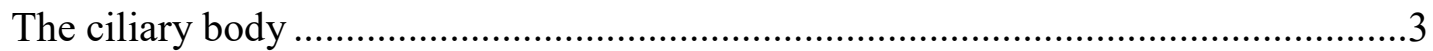

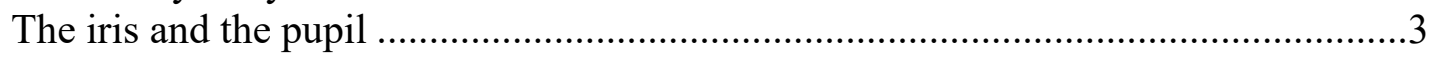

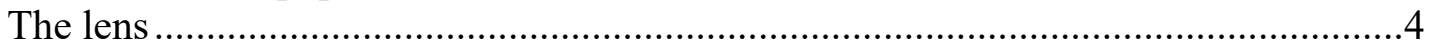

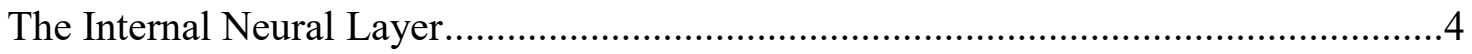

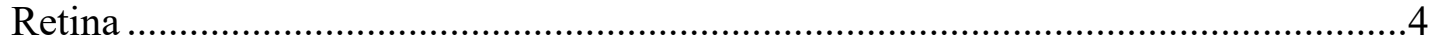

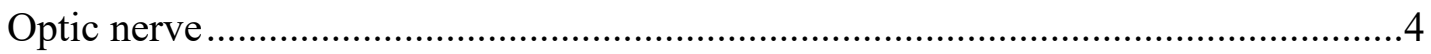

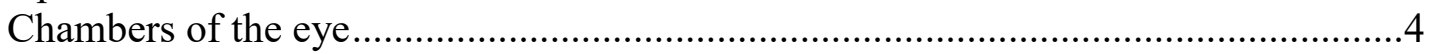

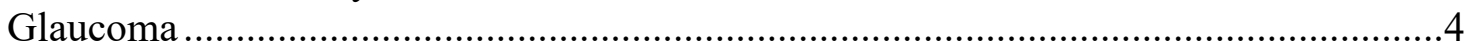

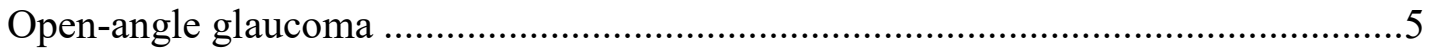

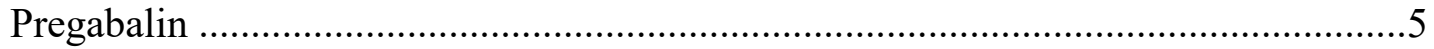

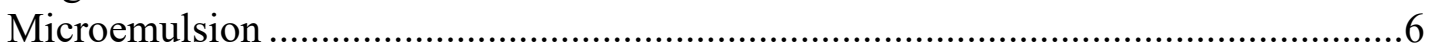

Research Hypothesis .......................................................................................

Aim 1: To optimize and develop a multiple $\mathrm{W} / \mathrm{O} / \mathrm{W}$ microemulsion eye drops .........8

Aim 2: To characterize the prepared multiple $\mathrm{W} / \mathrm{O} / \mathrm{W}$ microemulsion eye drops ......8

Aim 3: To determine the safety, in vivo efficacy and cell toxicity of our prepared multiple $\mathrm{W} / \mathrm{O} / \mathrm{W}$ microemulsion eye drops in Dutch belted rabbits and a human

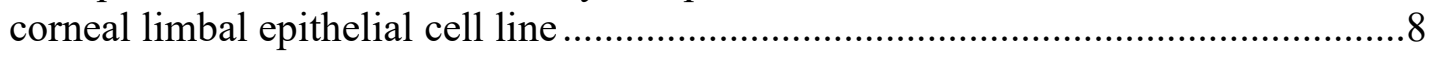

Aim 4: To determine the physical and chemical stability of our ME eye drops.........9

\section{CHAPTER 2. OPTIMIZATION AND DEVELOPMENT OF A MULTIPLE} WATER IN OIL IN WATER MICROEMULSION EYE DROPS ..........................10

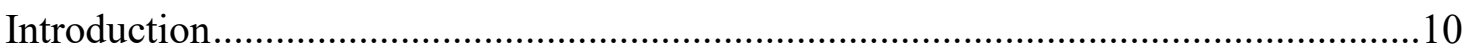

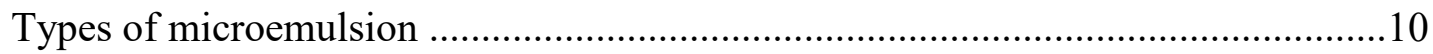

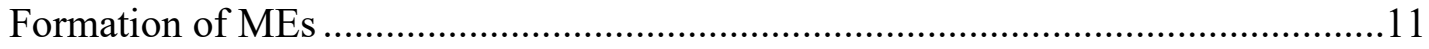

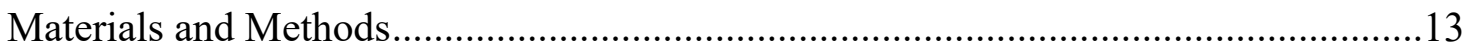

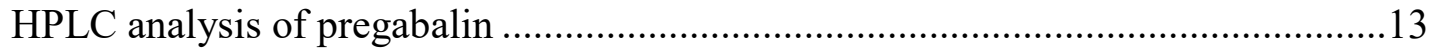

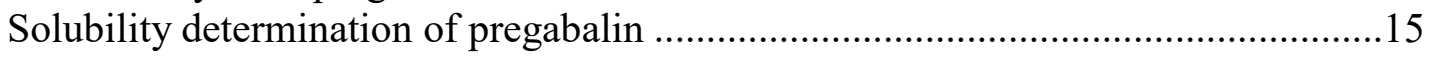

Preparation of the Multiple ME Eye Drops ........................................................ 15

Construction of ternary phase diagrams and preparation of the primary W/O ME ...15

Preparation of external aqueous phase and incorporation of the bioadhesive

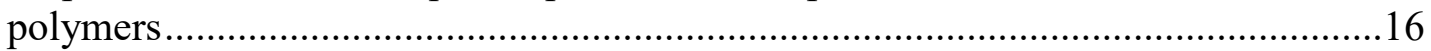

Preparation of the final multiple W/O/W ME ......................................................... 16

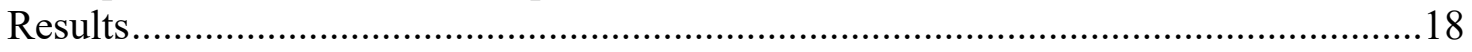


Our constructed ternary and pseudo-ternary phase diagrams ............................... 18

The external aqueous phase surfactant/co-surfactant mixture composition ..............20

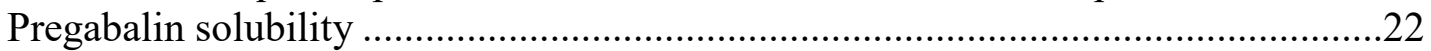

\section{CHAPTER 3. IN VITRO CHARACTERIZATION OF THE PREPARED} PREGABALIN-LOADED W/O/W ME EYE DROPS ................................................23

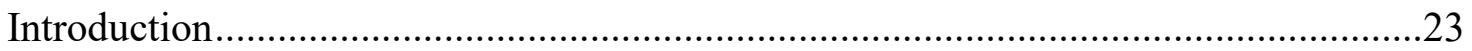

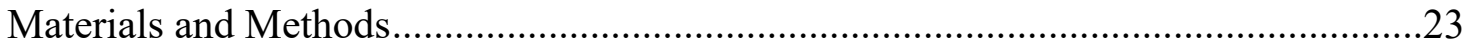

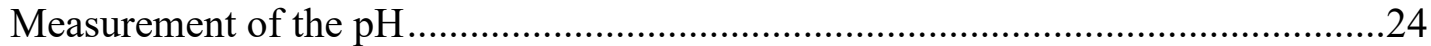

Average droplet size, polydispersity index (PDI) and zeta potential measurement ..24

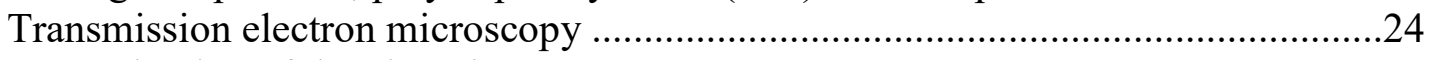

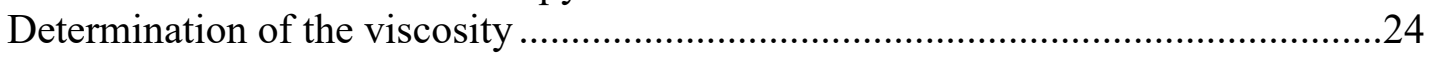

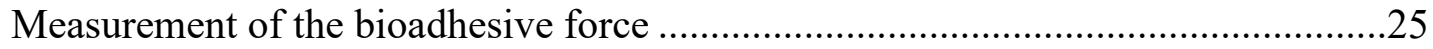

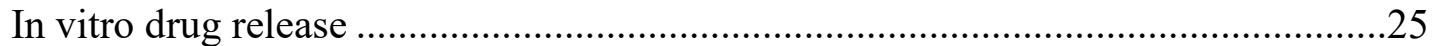

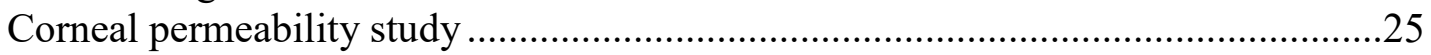

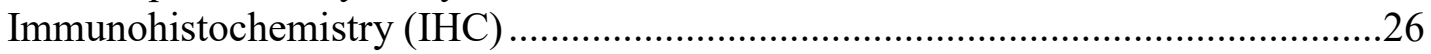

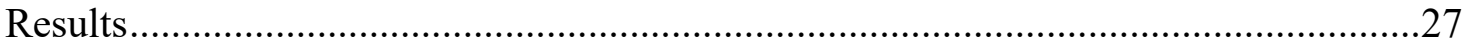

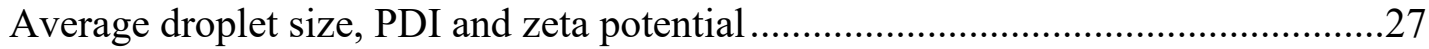

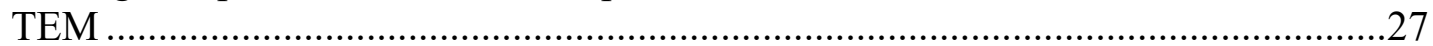

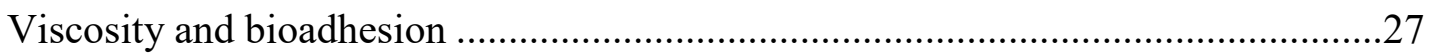

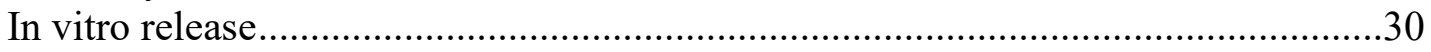

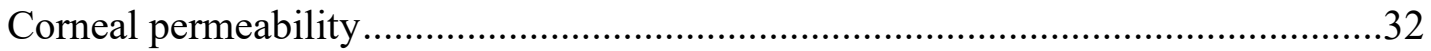

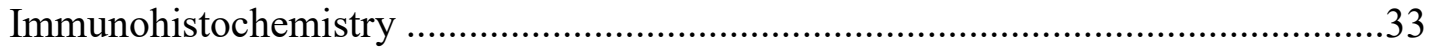

\section{CHAPTER 4. DETERMINATION OF SAFETY, IN VIVO EFFICACY AND}

THE CELL TOXICITY OF THE MICROEMULSION EYE DROPS ....................37

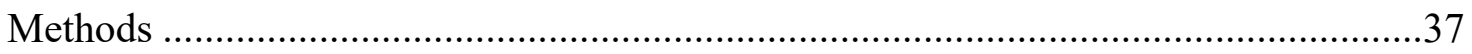

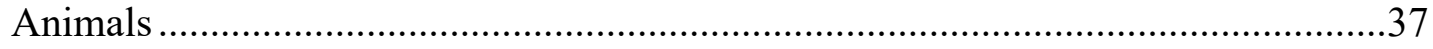

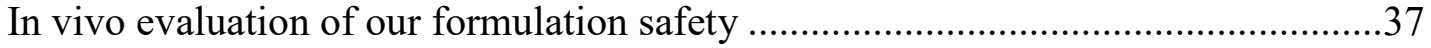

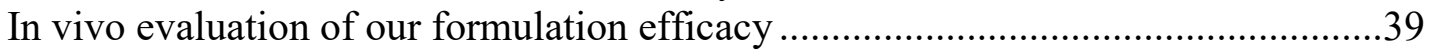

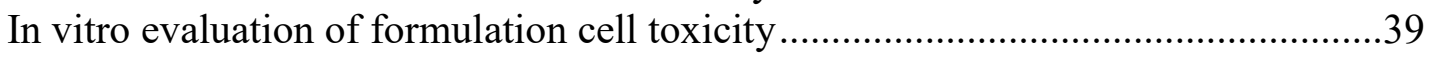

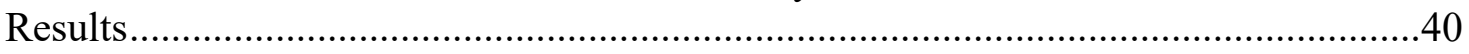

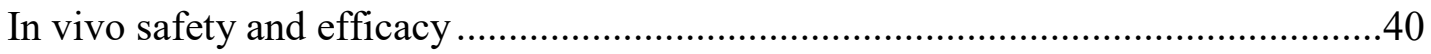

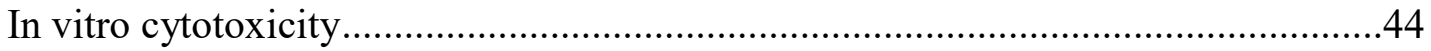

\section{CHAPTER 5. STABILITY STUDY OF PREGABALIN-LOADED CARBOPOL}

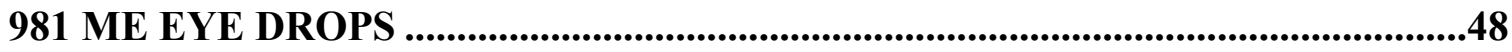

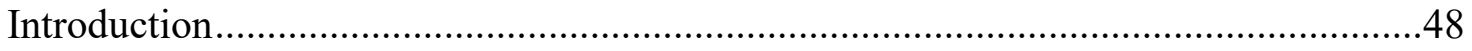

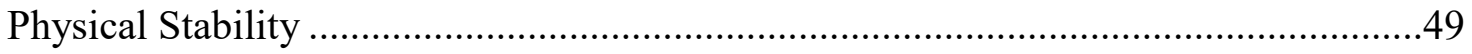

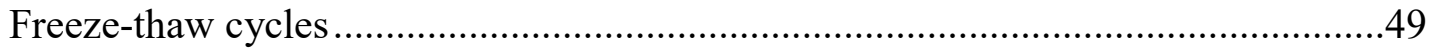

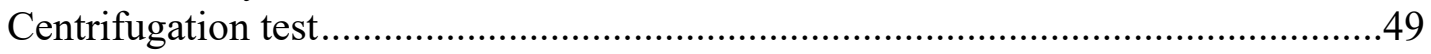

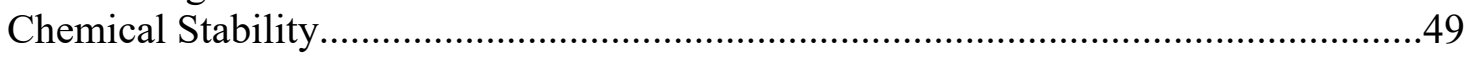

$\mathrm{pH}$ of the prepared multiple ME formulation ....................................................5

Drug content of the prepared multiple ME formulation ....................................50

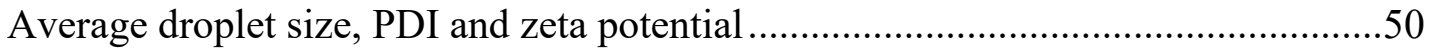

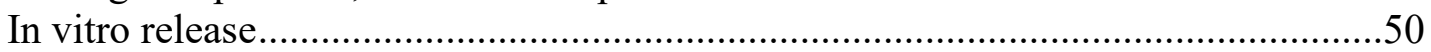




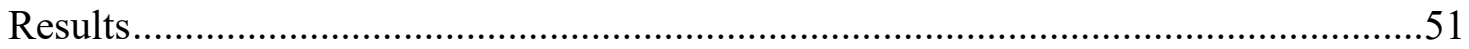

Physical stability ........................................................................................51

$\mathrm{pH}$ of our multiple ME eye drops .....................................................................

Average droplet size, polydispersity index (PDI) and zeta potential.........................51

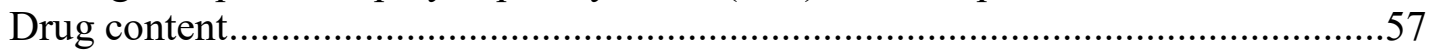

In vitro release

CHAPTER 6. DISCUSSION AND CONCLUSION......................................................64

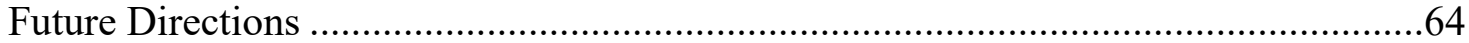

Aim 1: To optimize and develop a multiple W/O/W microemulsion eye drops .......64

Aim 2: To characterize the prepared multiple $\mathrm{W} / \mathrm{O} / \mathrm{W}$ microemulsion eye drops ....65

Aim 3: To determine the safety, in vivo efficacy and cell toxicity of our prepared multiple $\mathrm{W} / \mathrm{O} / \mathrm{W}$ microemulsion eye drops in Dutch belted rabbits and a human corneal limbal epithelial cell line.....

Aim 4: To determine the physical and chemical stability of our ME eye drops........68

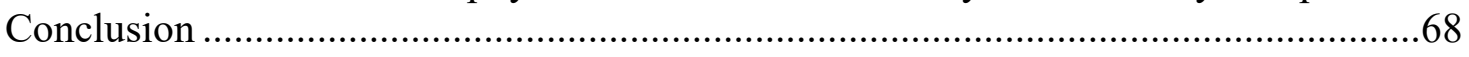

LIST OF REFERENCES ...................................................................................................70

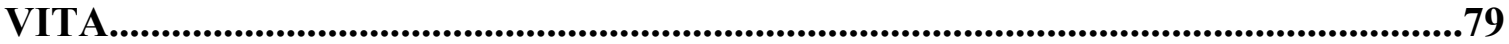




\section{LIST OF TABLES}

Table 2-1. Composition of pregabalin-loaded bioadhesive multiple W/O/W ME........17

Table 3-1. Droplet size, PDI and zeta potential of blank and medicated ME formulations

Table 3-2. In vitro transcorneal permeability parameters of pregabalin from multiple $\mathrm{W} / \mathrm{O} / \mathrm{W} \mathrm{ME}$ and control

Table 4-1. Pharmacodynamic parameters after application of $0.6 \%$ pregabalinloaded ME chitosan, sod. alginate, Carbopol 981 and control eye drops to Dutch belted rabbits

Table 4-2. Statistical comparisons among different pregabalin ME eye drops and the control after application of a single dose.

Table 5-1. The $\mathrm{pH}$ values of our developed pregabalin-loaded ME eye drops at different temperatures after four month of stability study

Table 5-2. Statistical comparisons among $\mathrm{pH}$ values of pregabalin-loaded Carbopol $\mathrm{ME}$ eye drops stored at four different temperatures and the initial $\mathrm{pH}$ value of the same formulation

Table 5-3. The average droplet size of our prepared pregabalin-loaded ME eye drops at different temperatures after four month of stability study

Table 5-4. Statistical comparisons among droplet size values of pregabalin-loaded Carbopol ME eye drops stored at four different temperatures and the initial droplet size value of the same formulation

Table 5-5. The average polydispersity index of our prepared pregabalin-loaded ME eye drops at different temperatures after four month of stability study .......56

Table 5-6. Statistical comparisons among PDI values of pregabalin-loaded Carbopol ME eye drops stored at four different temperatures and the initial droplet size value of the same formulation

Table 5-7. The average zeta potential of our prepared pregabalin-loaded ME eye drops at different temperatures after four month of stability study.

Table 5-8. Statistical comparisons among the zeta potential values of pregabalinloaded Carbopol ME eye drops stored at four different temperatures and the initial zeta potential value of the same formulation 
Table 5-9. The percentage amount remaining of pregabalin in our prepared pregabalin-loaded ME eye drops at different temperatures after four month of stability study

Table 5-10. Statistical comparisons among the percentage amount remaining of pregabalin values of pregabalin-loaded Carbopol ME eye drops stored at four different temperatures and the initial value of the same formulation...60

Table 5-11. Statistical comparisons among the cumulative amounts released of pregabalin after four months of stability and the initial values of the same formulation 


\section{LIST OF FIGURES}

Figure 1-1. Subunit assembly of voltage-gated calcium channels ....................................7

Figure 2-1. Schematic of the effect of temperature (T) or salinity (S) and the concentration of surfactant on ME type

Figure 2-2. Schematic of the energetics involved in a typical microemulsion disperse system

Figure 2-3. Ternary and pseudo-ternary phase diagrams of the primary W/O ME

Figure 2-4. Cartoon showing composition of pregabalin-loaded multiple W/O/W ME eye drops

Figure 3-1. TEM image of pregabalin-loaded ME

Figure 3-2. Viscosity and bioadhesion of pregabalin-loaded ME formulations and controls

Figure 3-3. Cumulative amount released (\%) of pregabalin from ME eye drops

Figure 3-4. Cumulative amount permeated of pregabalin from ME eye drops using rabbit cornea

Figure 3-5. Transcorneal flux of pregabalin from pregabalin-loaded ME eye drops......35

Figure 3-6. Cellular localization of CACNA2D1 in Dutch belted rabbits eye..................36

Figure 4-1. IOP reduction (\%) after application of pregabalin simple eye drops.............38

Figure 4-2. IOP reduction (\%) after $0.6 \%$ pregabalin ME eye drops application in Dutch belted rabbits

Figure 4-3. Slit lamp examination after application of pregabalin-loaded ME eye drops

Figure 4-4. Cytotoxicity histogram of pregabalin-loaded Carbopol ME eye drops using HCLE

Figure 5-1. The $\mathrm{pH}$ of pregabalin-loaded Carbopol ME eye drops at different temperatures after four month of stability study .

Figure 5-2. The mean droplet size of pregabalin-loaded ME eye drops at different temperatures after four month of stability study

Figure 5-3. The PDI of pregabalin-loaded Carbopol ME eye drops at different temperatures after four month of stability study 
Figure 5-4. The zeta potential of pregabalin-loaded Carbopol ME eye drops at different temperatures after four months of stability study ...........................58

Figure 5-5. Cumulative amount released (\%) of pregabalin from Carbopol ME eye drops $(0.6 \%)$ at different temperatures after four month of stability study..62 


\section{LIST OF ABBREVIATIONS}

$\begin{array}{ll}\text { AUC total } & \text { Area under the curve (total) } \\ \text { BCS } & \text { Biopharmaceutics classification system } \\ \text { CB } & \text { Ciliary body } \\ \text { DMSO } & \text { Dimethyl sulfoxide } \\ \text { HCLE } & \text { Human corneal limbal epithelial cells } \\ \text { HLB } & \text { Hydrophilic-lipophilic balance } \\ \text { ICH } & \text { International Conference on Harmonisation } \\ \text { IOP } & \text { Intraocular pressure } \\ \text { LOA } & \text { List of Abbreviations (sample 2 }{ }^{\text {nd }} \text { entry) } \\ \text { ME } & \text { Microemulsion } \\ \text { MTT } & \text { Methylthiazolyldiphenyl tetrazolium } \\ \text { O/W } & \text { Oil in water } \\ \text { PDI } & \text { Polydispersity index } \\ \text { POAG } & \text { Primary open-angle glaucoma } \\ \text { PRG } & \text { Pregabalin } \\ T_{\text {end }} & \text { Time required for IOP to return again to its baseline } \\ \text { TM } & \text { Trabecular meshwork } \\ T_{\text {max }} & \text { Time required to reach maximum decrease in IOP } \\ \text { W/O } & \text { Water-in-oil } \\ \text { W/O/W } & \text { Water-in-oil-in-water }\end{array}$




\section{CHAPTER 1. INTRODUCTION}

Topical application of drugs as eye drops (simple drug solutions) is the most advantageous route for ocular drug delivery to treat various eye diseases affecting the anterior segment of the eye. Because of the simplicity and the avoidance of the systemic absorption that led to a good patient acceptance, these conventional ophthalmic dosage forms account for nearly $90 \%$ of the currently available marketed ocular formulations (1). However the extensive and rapid precorneal loss of most of the applied volume of eye drops caused by the high tear fluid turnover and the rapid drainage limited the efficiency and the intraocular bioavailability of these topical formulations (2-4).

Ocular drug delivery is one of the most challenging research areas because of the unique structure of the eye and the specific environment available for drug application. It's very important to study the anatomy of the eye before designing an efficient new ocular drug delivery system as a therapy for various eye disorders.

\section{Anatomy of the Eye}

The eye consists of three main parts; eyebrows \& eyelids, the conjunctiva and the eyeball.

\section{Eyebrows and eyelids}

The eyebrows are well known for its importance for facial expression and human communication. Also, it helps to prevent the falling of debris, water and sweat down into

the eye socket. Coming to the eyelids as a protective cover for the eyeball, it also helps to keep its surface lubricated.

\section{The conjunctiva}

The conjunctiva is a thin semi-transparent moist mucous membrane that originates at the corneoscleral junction. Conjunctiva consists of two portions; the first one that covers the inner side of the eyelids (the palpebral conjunctiva) and the second portion that covers the front surface of the eyeball except the central clear cornea (the bulbar conjunctiva). It composed of multilayered epithelium on top of vascularised connective tissue (5). The conjunctiva contains many glands which are involved in normal physiological processes such as the accessory glands of Wolfring and Krause. The function of the conjunctiva is to produce mucous as well some components of the tear film (6). 


\section{The eyeball}

It is mainly made up of three layers:

1. The external fibrous layer that includes sclera, cornea, limbus, trabecular meshwork and canal of Schlemm.

2. The intermediate vascular/muscular layer that consists of two parts; (iris-ciliary body) and posterior (choroid).

3. The internal neural layer (the sensory part of the eye) includes chambers of the eye, retina, optic nerve and macula.

\section{The External Fibrous Layer}

This outer layer of the eyeball consists of relatively tough fibrous tissues made up of collagen and elastin. It comprises:

\section{The sclera (the white of the eye)}

It is a dense, opaque white, fibrous structure constitutes the posterior five- sixth of the globe It consists of three layers; episclera, sclera proper and lamina fussa. It contains few blood vessels and provides the structural integrity of the eye.

\section{The cornea}

It is the transparent tissue that covers the pupil, the iris and lens. The thickness of the healthy cornea is $0.5-0.8 \mathrm{~mm}$ with a radius of $11-12 \mathrm{~mm}$. Because of the difference in the structural organization of the collagen fibers in the cornea and the sclera, one is clear while the other is opaque, respectively. Collagen fibers are arranged in highly regular laminae while it appear interwoven and extend in all directions in the sclera (7). Morever, there are no blood vessels in the cornea and oxygen is mainly provided by diffusion so it is transparent for optimal visual acuity (6). Furthermore, the function of the cornea is to control and focus the light entering the eye by working as a window. The cornea is composed of five layers: the outermost epithelium, Bowman's layer, the middle corneal stroma, Descemet's membrane, and innermost endothelium. The corneal epithelium is the rate-limiting barrier that restricts the permeation of hydrophilic and large molecules because of the tight junctions in the most superficial cells $(8,9)$. The corneal epithelium is covered by the pre-corneal tear film, which ensures nutrition of the anterior surface of the cornea. The permeation of very lipophilic drugs may be restricted by the highly permeable hydrophilic stroma due to the limited partitioning from the epithelium to the stroma $(8,10)$. Descemet's membrane is the acellular basement membrane of the corneal endothelium containing mainly type IV collagen. 
The corneal endothelium is a monolayer that regulates fluid transport from the aqueous humor. In ocular drug absorption, the corneal endothelium is not considered a significant barrier. For topically applied drugs the cornea and the conjunctiva are the major tissue barriers that restrict their ocular absorption but with a variable contribution (11).

Limbus is a transitional zone 1-2 mm wide between the cornea, the sclera and conjunctiva. It includes the trabecular meshwork and the canal of Schlemm. The trabecular meshwork surrounds the circumference of the anterior chamber. It is a porouslike structure with opening diameter $2-3 \mu \mathrm{m}$ through which the aqueous humor flows into the canal of Schelmm which is an oval-shaped channel that covers the entire circumference of the anterior chamber. It connected to the anterior chamber with the trabecular meshwork.

\section{The Intermediate Vascular/Muscular Layer}

This layer located inside the fibrous layer.

\section{The choroid}

The choroid is a rich vascular layer that supplies oxygen and nutrients to the outer layers of retina and the structures of the anterior chamber. It extends from the optic nerve posteriorly to the ciliary body anteriorly.

\section{The ciliary body}

The ciliary body extends from the iris to the choroid. It is divided into two portions; the uveal portion and the epithelial portion. The main functions of the ciliary body are to secrete aqueous humor and to allow the eye to adjust distant objects in order to obtain a clear image on the retina.

\section{The iris and the pupil}

The iris is the colored tissue of the eye. It is located in front of the lens and the ciliary body. It separates the anterior and posterior chambers. Its main function is to regulate the amount of the light entering the eye via dilation and constriction to ensure clear vision (6). A central opening in the iris is the pupil which controls the amount of light admitted to the eye. The color of the iris that is responsible for eye's color depends on the amount of melanin in its tissues. 


\section{The lens}

The lens is a crystalline, transparent biconvex structure located behind iris and the pupil aperture and in front of the vitreous body. It is suspended by zonular fibers and is about $4 \mathrm{~mm}$ thick and $10 \mathrm{~mm}$ diameter. It does not have any blood vessels, nerve fibers, or connective tissue, and is nourished by the aqueous humor surrounding it.

\section{The Internal Neural Layer}

It's the innermost layer of the eyeball. It has many layers of cells including retina, optic nerve, chambers of the eye and macula.

\section{Retina}

It is a complex, densely interconnected, 10 layered tissue which is an extension of the central nervous system. It contains two types of photoreceptor cells-rods and cones - which transfer impulses from the retina to the brain visual cortex via the optic nerve. Rods are responsible for black and white vision during dim light. While, cones are responsible for seeing colored objects at day light.

\section{Optic nerve}

The optic nerve is a portion of the central nervous system consisting of axons of retinal ganglion cells together with nerve fibers that extend from the brain visual cortex to the eye.

\section{Chambers of the eye}

Internally the eye is made up of two unequal compartments: a smaller compartment that lies anterior to the eye lens termed the aqueous compartment, and a larger (vitreous) compartment that lies behind the lens. The aqueous compartment is further divided into an anterior and a posterior chamber by the iris (Presland, 2007).

Macula is a small specialized yellow pigmented zone in the retina with a diameter of $7 \mathrm{~mm}$. It is responsible for central or reading vision. Its main function is to allow to see fine and to protect the eye from the high energy blue light (Dentchev et al., 2007).

\section{Glaucoma}

Glaucoma is a multifactorial eye disease that can cause optic nerve damage and irreversible blindness, which considered a significant public health problem worldwide. It 
can be defined as a group of ocular diseases that may cause characteristic, progressive changes in the optic nerve head, visual field loss, or both $(12,13)$. There are several types of glaucoma while among all these types, primary open-angle glaucoma (POAG) is the most common form that accounts for at least $90 \%$ of all glaucoma cases all over the world $(14,15)$. Treatment of glaucoma depends upon the nature and severity of each case. In general, glaucoma cannot be cured, but it can be controlled. Topical eye preparations, oral preparation, laser procedures, and surgical operations are used to prevent or slow further damage. Also, with any type of glaucoma, regular eye examinations are very important to detect progression and to prevent vision loss (16).

\section{Open-angle glaucoma}

It is also called primary or chronic glaucoma. The term open-angle means that the angle where the iris meets the cornea is patent. Elevated IOP is one of the most significant risk factors contributing to visual field loss in primary open angle glaucoma. IOP is generated by the balance of aqueous humor production by the ciliary body (CB) and its drainage through the trabecular meshwork (TM) (conventional pathway), and to a lesser degree the unvisceral or nonconventional pathway. An imbalance between the inflow and outflow of aqueous humor leads to a change in IOP $(17,18)$. Open-angle glaucoma has several characteristics. It is a lifelong condition that develops very slowly. In addition, its symptoms and resultant damage are usually not noticed by the patient until significant visual field loss has occurred. Lastly, it has a wide angle between the iris and the cornea (19). The only treatment of open-angle glaucoma, aside from surgery, is IOP-reducing drugs applied topically onto the cornea. Unfortunately, several daily applications of such drugs are necessary to maintain reduced IOP, which can be accompanied by poor patient satisfaction/compliance and potential toxicity to the anterior structures of the eye.

Despite the availability of several POAG approved drugs, such as carbonic anhydrase inhibitors, prostaglandins, and beta-blockers, yet these agents are not a cure. They are not universally effective in reducing IOP and are laden with various side effects. Accordingly, patient compliance is low and visual deterioration is frequent, resulting in severe impairment and blindness in millions of people around the world. So the availability of an effective drug with minimal or no side effects is still urgent unmet medical need.

\section{Pregabalin}

It is (S)-3-aminomethyl-5-methylhexanoic acid, a gabapentinoid drug. It has been clinically effective in treating epileptic seizures or neuropathic pain disorders such as fibromyalgia and diabetic peripheral neuropathy $(20,21)$. Pregabalin has become a lead molecule due to its hydrophilicity, rapid absorption after oral administration and high bioavailability $(\sim 90 \%)(22)$. It is currently marketed by Pfizer (NY, USA) as Lyrica ${ }^{\circledR}$. According to the biopharmaceutics classification system (BCS) pregabalin is categorized 
as a class I drug (highly soluble and highly permeable) (23). Pregabalin binds with high affinity and high selectivity to the CACNA2D1 subunit of the voltage-dependent calcium channels (Figure 1-1) in eye tissues (trabecular meshwork and ciliary body) and lowers IOP in POAG (24).

Of course, eye drops are the most commonly used and acceptable topical ophthalmic dosage form all over the world $(1,25)$. When the active ingredient is a watersoluble drug, this dosage form usually consists of a simple drug solution. The prevalence of this dosage form may be due to the ease of manufacturing and the ease of application into the eye. Unfortunately, this dosage form suffers from several drawbacks that may result in absorption of only $5 \%$ of the administered dose into the eye tissues $(26,27)$. These drawbacks include, the rapid drainage from the eye surface either to the outside of the eye or to the systemic circulation through nasolacrimal duct and the very short corneal contact time (28). Additionally, the high blood perfusion of theconjunctiva leads to systemic absorption of a large fraction of the drug that was absorbed through this tissue. Several daily applications may overcome these drawbacks, yet they usually result in exaggeration of the systemic side effects and lower patient satisfaction and compliance that may cause the patient to discontinue the drug use and worsen his/her visual loss.

Several formulations strategies have been used to overcome the drawbacks associated with aqueous solution eye drops among them; viscosity-inducing agent (2931 ), penetration enhancers (32), bioadhesive polymers (33), ocular implants $(26,34,35)$, contact lenses $(36,37)$, ocular inserts $(38)$, ocular injections $(39,40)$ and colloidal drug delivery systems such as liposomes, nanosuspensions, microparticles, nanoparticles, nanoemulsions and microemulsions (41). All the listed systems may sustain drug release and/or prolong the corneal contact time. Among all these systems, microemulsions offer several advantages that make them superior to other colloidal drug delivery systems.

\section{Microemulsion}

Microemulsion (ME) can be defined as a single-phase, optically clear, isotropic, thermodynamically stable, system with droplet size in the submicron range $(10-100 \mathrm{~nm}$ diameter) (42-45). MEs are stabilized by a surfactant which is an amphiphilic compound usually used in combination with a co-surfactant, which may be an amine, a short chain alcohol or another weakly amphiphilic molecule $(46,47)$.

MEs are considered one of the most promising carriers for ocular drug delivery by offering several advantages including thermodynamic stability (48), optical clearance, ease of preparation and scaling up (49), few synthesis steps, small energy requirement during their preparation, $100 \%$ encapsulation efficiency, compatibility with both watersoluble and water-insoluble drugs and improved bioavailability through facilitating drug transcorneal permeability (50).

MEs are considered one of the most promising carriers for ocular drug delivery by offering several advantages including thermodynamic stability (48), optical clearance, 


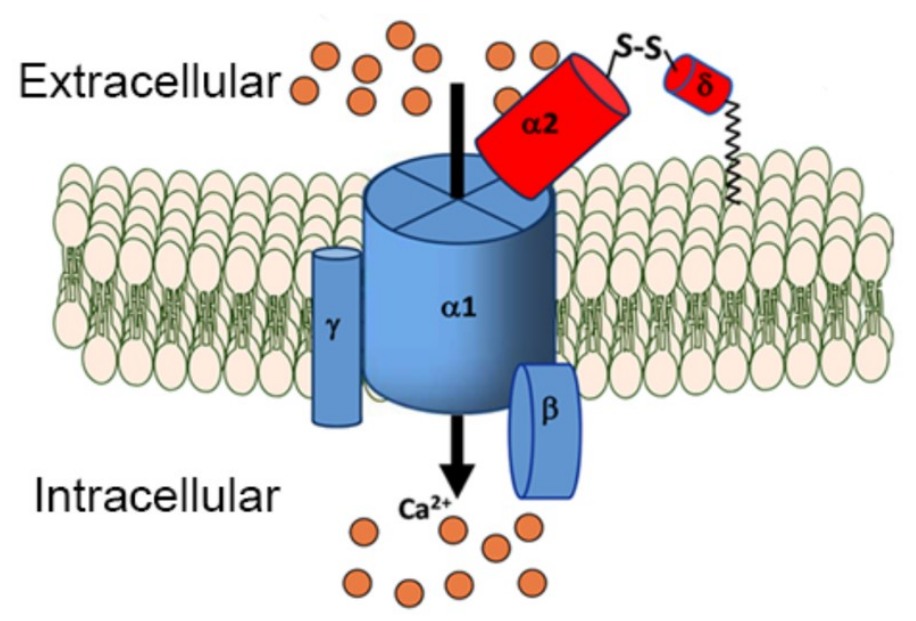

Figure 1-1. Subunit assembly of voltage-gated calcium channels

Graphic representation of the high voltage-activated calcium channel complex consisting of the main pore forming $\alpha_{1}$ (blue) subunit plus ancillary, $\beta$ (blue), $\gamma$ (blue), and $\alpha_{2}$ (red) and $\delta_{1}$ (red) subunits. $\alpha 2$ and $\delta_{1}$ subunits have a disulphide bond between them. Reprinted with permission. Chintalapudi SR, Maria D, Di Wang X, Bailey JNC, consortium N, International Glaucoma Genetics c, et al. Systems genetics identifies a role for Cacna2d1 regulation in elevated intraocular pressure and glaucoma susceptibility. Nat Commun. 2017;8(1):1755. doi: 10.1038/s41467-017-00837-5. 
ease of preparation and scaling up (49), few synthesis steps, small energy requirement during their preparation, $100 \%$ encapsulation efficiency, compatibility with both watersoluble and water-insoluble drugs and improved bioavailability through facilitating drug transcorneal permeability (50).

Our recently published work showed that pregabalin generates an IOP-lowering response in both mice and rabbits after instillation of pregabalin simple eye drops (24). Unfortunately, the hydrophilicity of the drug may hinder its ophthalmic use, as it may lower its ocular bioavailability when applied in the form of an aqueous drug solution. In this study, we seek to overcome this problem by incorporating pregabalin in the innermost layer of our multiple ME, which will delay its release as it has to pass through several interfaces of the ME and the outermost layer includes a bioadhesive polymer. Based on this strategy, we predict that our new formulations could sustain the release of pregabalin for one day and maintain the IOP at lower level.

\section{Research Hypothesis}

We hypothesized that a new topical microemulsion bioadhesive ophthalmic formulation can sustain the release of pregabalin as a novel glaucoma therapy. The studies presented in Chapters 2-5 address the following specific aims:

\section{Aim 1: To optimize and develop a multiple $\mathrm{W} / \mathrm{O} / \mathrm{W}$ microemulsion eye drops}

1. What is the proper surfactant mixture and its ratio that shows the largest ME region in the pseudo-ternary phase diagram?

2. How we can optimize and develop a multiple water-in-oil-in-water ME eye drops by incorporating a bioadhesive polymer in the external aqueous phase?

\section{Aim 2: To characterize the prepared multiple $\mathrm{W} / \mathrm{O} / \mathrm{W}$ microemulsion eye drops}

1. Can we sustain the in vitro release of pregabalin by incorporation of the drug in the innermost layer of the developed multiple $\mathrm{W} / \mathrm{O} / \mathrm{W}$ ME?

2. Can we determine how our developed formulations affect the corneal permeability of pregabalin compared to the control?

\section{Aim 3: To determine the safety, in vivo efficacy and cell toxicity of our prepared multiple $\mathrm{W} / \mathrm{O} / \mathrm{W}$ microemulsion eye drops in Dutch belted rabbits and a human corneal limbal epithelial cell line}

1. Can we determine the safety and in vivo efficacy of our prepared multiple W/O/W ME eye drops in Dutch belted rabbits after instillation of a single drop of the formulations? 
2. Do our developed ophthalmic formulations have any level of cell toxicity to a human corneal limbal epithelial cell line?

\section{Aim 4: To determine the physical and chemical stability of our ME eye drops}

1. Do our multiple ME eye drops will remain physically stable after both freezethaw cycles and centrifugation tests?

2. What is the shelf life and the expiration date of our ME eye drops after a longterm and an accelerated stability study at four different temperature? 


\section{CHAPTER 2. OPTIMIZATION AND DEVELOPMENT OF A MULTIPLE WATER IN OIL IN WATER MICROEMULSION EYE DROPS}

\section{Introduction}

In emulsion systems, MEs exhibit the smallest droplet sizes (10-100 $\mathrm{nm}$ in diameter) compared to both macro- and nanoemulsions. MEs are considered a unique colloidal drug delivery system for various routes of administration (51). It has the ability to deliver both hydrophilic and lipophilic (52) drugs with improved bioavailability and stability. Also, these systems are formed spontaneously (53) without the need of energy input offering a very easy and cheap production process (54).

There are four classes of surfactants, non-ionic (Tweens), anionic (sodium dodecyl benzene sulfonate), cationic (quaternary ammonium salt) and zwitterionic (amphoteric e.g lecithin). The formation of MEs requires a relatively high concentration of surfactants (i.e., $15-30 \% \mathrm{w} / \mathrm{w}$ of the oil phase) in contrast to nanoemulsions that require less surfactant $(1-3 \%$ of the volume of the oil phase) $(55,56)$. A surfactant works mainly through reducing the interfacial tension between the oil and water phases and hence lowers the energy required to increase the surface area. Non-ionic surfactants are more preferred in ME formulation due to their safety profile compared to ionic surfactants. In addition, non-ionic surfactants are more resistant to $\mathrm{pH}$ changes in comparison to other classes of surfactants (57).

\section{Types of microemulsion}

According to Winsor classification there are four general types of MEs $(58,59)$ :

1. Type I - biphasic ME with the surfactant preferentially soluble in water and oil-inwater $(\mathrm{O} / \mathrm{W})$ MEs form. However, in this Winsor I type of ME there is an excess upper oil phase where surfactant is only present as monomers at the interface that separated this oil phase with surfactant-rich aqueous phase.

2. Type II - biphasic ME where the surfactant is mainly soluble in the oil phase and water-in-oil (W/O) MEs form. Meanwhile, in this Winsor II type of ME an excess aqueous phase coexists with the surfactant-rich oil phase.

3. Type III - triphasic ME where surfactant-rich middle bicontinuous ME coexists with both the upper excess oil phase and the lower excess water phase (surfactantpoor phases) $(44,59)$.

4. Type IV- monophasic, it's a single phase micellar solution. This type of ME is spontaneously formed upon addition of sufficient concentration of surfactant/cosurfactant mixture. 
Depending on the emulsifier used, MEs can transition between each type quite easily by increasing the temperature in case of non-ionic surfactant or a simple change in salinity (electrolyte concentration) for ionic surfactant. In general, MEs follow trends displayed in Figure 2-1, typically referred to as a fish diagram (45).

A multiple ME is a multiple dispersion system where both hydrophobic and hydrophilic surfactants are incorporated during the preparation steps. So, it could be considered as ME of ME. There are two types of multiple ME:

1. Water-in-oil-in-water (W/O/W) ME: This multiple type of ME contains both $\mathrm{W} / \mathrm{O}$ and $\mathrm{O} / \mathrm{W}$ simple ME. Thus, the disperse system have small water droplets that are entrapped within larger oil droplets that in turn are dispersed in a continuous external aqueous phase. Furthermore, this unique structure of multiple ME make it very appropriate to sustain the release of water soluble drugs that are incorporated in the internal water droplets as it will act as a reservoir for the drug.

2. Oil-in-water-in-oil $(\mathrm{O} / \mathrm{W} / \mathrm{O}) \mathrm{ME}$ : This type of multiple $\mathrm{ME}$ contains both $\mathrm{O} / \mathrm{W}$ and $\mathrm{W} / \mathrm{O}$ simple ME. Thus, the disperse system have small oil droplets that are entrapped within larger water droplets that in turn are dispersed in a continuous external oil phase.

The hydrophilic-lipophilic balance (HLB) of a non-ionic surfactant is one of the most important parameters that should be considered for the proper selection of surfactants for a specific type of ME formulation during the early screening stage (60). The HLB can be calculated based on the structure of the surfactant with respect to the relative proportions of both hydrophilic and hydrophobic moieties within the molecule (61). A high HLB $(>10)$ of a surfactant indicates high hydrophilicity that will be optimal for $\mathrm{O} / \mathrm{W}$ microemulsion formation. Also, a hydrophobic surfactant with a low HLB $(\leq 5)$ is used during the preparation of $\mathrm{W} / \mathrm{O}$ microemulsion.

During the preparation of MEs, co-surfactants are used to help to decrease the concentration of the surfactant required during the formulation as it increase the fluidity of the interface and decrease the interfacial tension through improved packing (62). Glycols are the most commonly used co-surfactant with a carbon chain length ranging from C2-C10 (e.g propylene glycol). Also, alcohols with low molecular weight (nbutanol) and amines with short carbon chain are used as co-surfactants (59).

\section{Formation of MEs}

The formation of most MEs occurs spontaneously in the presence of the proper type and the specified concentration of a surfactant/co-surfactant without energy input $(54,63,64)$. Hence, the resulted colloidal dispersion is thermodynamically stable due to the very low interfacial tension between the oil phase and the aqueous phase (65). Also, as energy is not required for ME formation, the ME production in a large scale will be highly economic. The energy profile of MEs showed that the separated phases (i.e. oil 


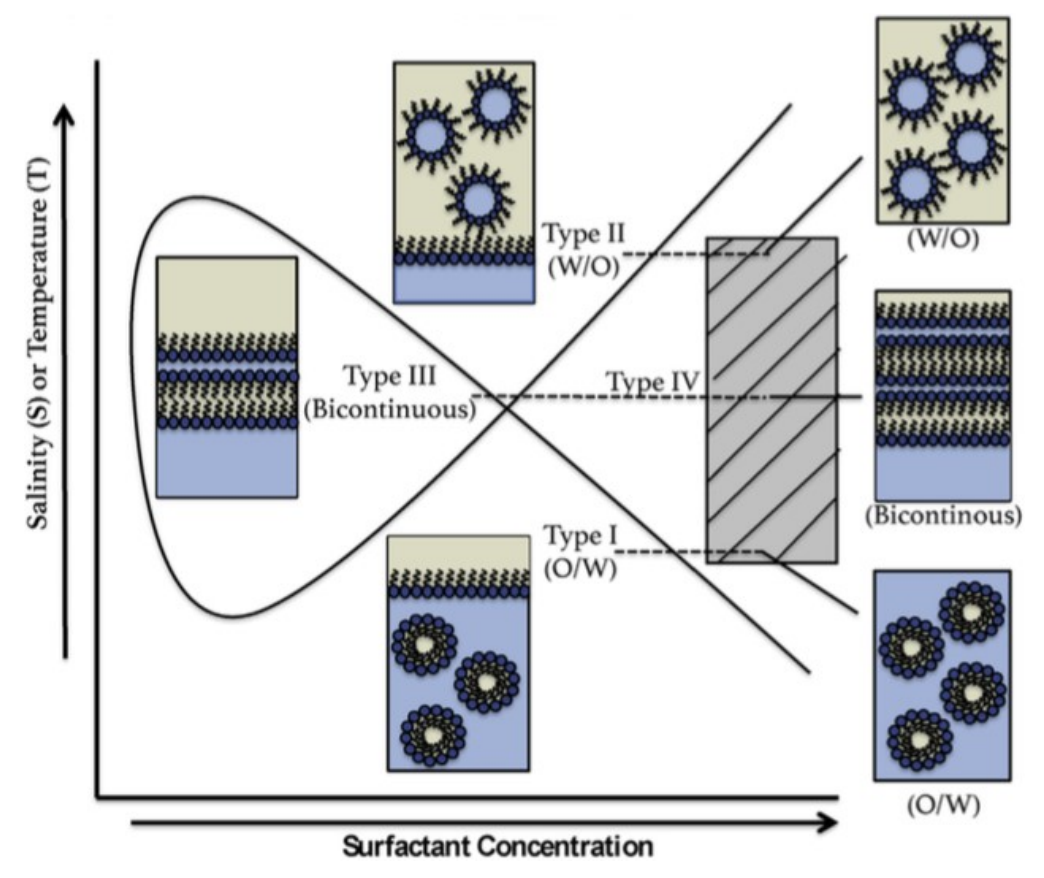

Figure 2-1. Schematic of the effect of temperature (T) or salinity (S) and the concentration of surfactant on ME type

MEs can transition between each type quite easily by increasing the temperature in case of non-ionic surfactant or a simple change in salinity (electrolyte concentration) for ionic surfactant.

Reprinted from Int J Pharm, 526(1-2), Callender SP, Mathews JA, Kobernyk K, Wettig SD, Microemulsion utility in pharmaceuticals: Implications for multi-drug delivery, 425442, copyright (2017), with permission from Elsevier. 
and aqueous phases) are at higher energetic state than that of the final ME product (Figure 2-2). Thus, energy is required for ME breaking into its original components.

For many years, MEs were reported to remain stable without showing any forms of instability (coalescence, creaming and breaking) $(47,66)$. In contrast, macroemulsion formulations have a strong tendency for creaming, flocculation and coalescence as a form of thermodynamic instability (67). Lv et al., 2005 reported that they were able to successfully increase the chloramphenicol stability by incorporating the drug into an $\mathrm{O} / \mathrm{W}$ ME ocular drug delivery system compared to the commercial eye drops (68).

Poor biopharmaceutical properties of more than $40 \%$ of drugs including poor water solubility hinder their marketing. In case of water-insoluble drugs, MEs can bring them into solution by dissolving them in suitable oil, which is then emulsified into water $(\mathrm{O} / \mathrm{W})$ to give a stable clear aqueous solution of such water-insoluble drug $(50,69,70)$. On the other hand, if the drug is water-soluble and suffers from rapid absorption and/or removal from the biological membranes, MEs can sustain their release for longer time.

The aim of this present work was to develop pregabalin-loaded multiple $\mathrm{W} / \mathrm{O} / \mathrm{W}$ bioadhesive ME stabilized by a combination of non-ionic hydrophilic and hydrophobic surfactants by the construction of ternary and pseudo-ternary phase diagrams.

\section{Materials and Methods}

Pregabalin ( $\geq 97 \%$ purity), pluronic $\AA$ F-68 (poloxamer 188; polyoxyethylenepolyoxypropylene block copolymer), chitosan (low molecular weight, 75-85\% deacetylated), glacial acetic acid, Cremophore ${ }^{\circledR}$ EL (Kolliphor ${ }^{\circledR}$ EL, polyoxyl castor oil) were purchased from Sigma-Aldrich (St. Louis, MO). Propylene glycol, Tween 80 (polysorbate 80), phosphoric acid, methanol and acetonitrile (HPLC grade) were purchased from Fisher Scientific (Fair Lawn, NJ). Gift samples of Labrafac lipophile WL1349, Capryol 90, transcutol P and Labrasol were obtained from Gattefossé Corporation (Paramus, NJ). Soybean L- $\alpha$-Lecithin (97.7\% phosphotidyl choline) was purchased from Calbiochem (Billerica, MA). Ethyl alcohol was purchased from Decon Labs, Inc. (King of Prussia, PA). Sodium alginate (viscosity of $1 \%$ solution at $25^{\circ} \mathrm{C}=5-$ $40 \mathrm{cP}$ ) was purchased from MP Biomedicals (Solon, OH). Carbopol 981 was obtained as a gift sample from Lubrizol advanced materials, Inc. (Cleveland, $\mathrm{OH}$ ).

\section{HPLC analysis of pregabalin}

A previously published reversed phase HPLC method was used for quantification of pregabalin through all experiments (71). Agilent 1100 series HPLC instrument (Germany) was equipped with Supelco kromasil C18 column $\left(5 \mu \mathrm{m}, 100^{\circ} \mathrm{A}, 4.0 \mathrm{~mm} \mathrm{x}\right.$ $300 \mathrm{~mm}$ ) that was operated at $25^{\circ} \mathrm{C}$. The mobile phase consisted of a mixture of methanol: acetonitrile: $0.02 \mathrm{M}$ dipotassium hydrogen orthophosphate in the ratio of $3: 1: 16, \mathrm{v} / \mathrm{v} / \mathrm{v}$ at a flow rate of $1 \mathrm{ml} / \mathrm{min}$. The effluent was monitored by photodiode array 


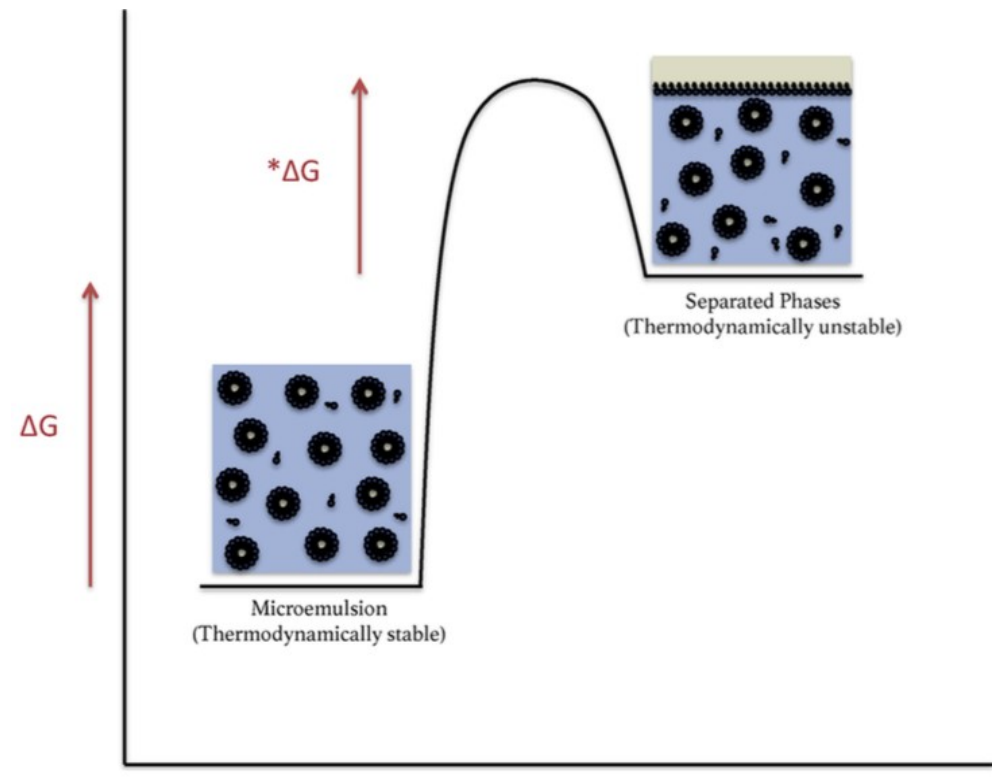

Figure 2-2. Schematic of the energetics involved in a typical microemulsion disperse system

The ME is thermodynamically stable because the ME product is at a lower energy state than that of its separated phases or starting components. However, nanoemulsions have a reversed profile with the nanoemulsion product at a higher energy state than the starting components.

Reprinted from Int J Pharm, 526(1-2), Callender SP, Mathews JA, Kobernyk K, Wettig $\mathrm{SD}$, Microemulsion utility in pharmaceuticals: Implications for multi-drug delivery, 425442, copyright (2017), with permission from Elsevier. 
detector and pregabalin was detected after a retention time of $5.4 \mathrm{~min}$ at a detection wavelength of $210 \mathrm{~nm}$.

\section{Solubility determination of pregabalin}

Solubility screening of pregabalin in different media as well as the non-aqueous ingredients of our ME was carried out in order to determine its maximum solubility in each ingredient (72). The used media were including: Labrafac lipophile WL 1349, (Capryol $90 \&$ soybean lecithin, 1:1 weight ratio mixture), water, 0.01N HCL and 0.01N $\mathrm{NaOH}$. An excess amount of the drug was added to $2 \mathrm{ml}$ of each medium in screw capped glass bottles. Suspensions were shaken at $180 \mathrm{rpm}$ for 7 days in a thermostatically controlled shaker at $25 \pm 0.5^{\circ} \mathrm{C}$. The obtained suspensions were allowed to equilibrate for further one day. After equilibration, suspensions were filtered through $0.45 \mu \mathrm{m}$ membrane filters (Millipore, Billerica, MA) and the filtrate was suitably diluted with the mobile phase then assayed for its drug content by HPLC. In case of oil and surfactant media, the filtrate was diluted with absolute ethanol before HPLC assay. Each experiment was performed in triplicate and the results were calculated as mean $\pm \mathrm{SD}$.

\section{Preparation of the Multiple ME Eye Drops}

Preparation of our multiple ME eye drops was achieved by emulsification method in three steps. The first step included the formation of the primary W/O ME. The second step involved the preparation of the bioadhesive external aqueous phase. Finally, the primary W/O ME was further emulsified by drop wise addition into the bioadhesive external aqueous phase to produce the final bioadhesive multiple ME eye drop

\section{Construction of ternary phase diagrams and preparation of the primary $\mathrm{W} / \mathrm{O} \mathrm{ME}$}

The primary W/O ME usually consists of an oil phase, aqueous phase, and single surfactant or surfactants mixture (HLB less than 7). To determine the appropriate ratio of each component that can efficiently produce a ME, we constructed several ternary phase and pseudo-ternary phase diagrams using Labrafac lipophile WL1349 as oil phase, MilliQ water as aqueous phase and a single surfactant (in case of ternary phase diagram) or surfactants mixture (in case of pseudo-ternary phase diagram). The used surfactants were Capryol 90, transcutol P, soybean lecithin, or different combination of them.

The ternary phase diagrams were accurately generated using a water titration method $(50,51)$. In this method, oil was mixed carefully with surfactant or surfactants mixture in diverse weight ratios from 0.5:9.5 to $9.5: 0.5(\% \mathrm{~W} / \mathrm{W})$ by vortex for $2 \mathrm{~min}$ at $25^{\circ} \mathrm{C}$. Then the produced mixture was slowly titrated with aliquots of MilliQ water until the appearance of the first turbidity, which reflected the boundary point that differentiated the end of the water-in-oil (W/O) ME region and the beginning of macroemulsion region. To present the four ingredients in the pseudo-ternary phase diagram, the top apex of the 
triangle represents the oil while the other apices represent surfactant/co-surfactant and the aqueous phase (73).

Ternary or pseudo-ternary phase diagram that showed the largest $\mathrm{ME}$ area was selected to pick up one point to prepare the primary $\mathrm{W} / \mathrm{O} \mathrm{ME}$ to be used for further investigations. The point that picked up was consisted of $20 \%$ water $+30 \%$ Labrafac lipophile WL1349 + 50\% (Capryol 90 \& soybean lecithin, 1:1 mixture).

\section{Preparation of external aqueous phase and incorporation of the bioadhesive polymers}

For preparation of the external aqueous phase, several surfactants with high HLB values $(>12)$ were screened such as Tween 80 , Cremophor ${ }^{\circledR}$ EL (74), poloxamer 188, brij 97 and Labrasol. Aqueous solutions of different concentrations of these surfactants were prepared and tested by titration with the previously prepared primary W/O ME until the appearance of the first turbidity.

The surfactant solution that could incorporate the greatest amount of the primary W/O ME was selected for further investigations. In order to increase the amount of incorporated $\mathrm{W} / \mathrm{O} \mathrm{ME}$, the selected surfactant was mixed with other surfactants and/or co-surfactant.

After surfactants screening, the external aqueous phase was prepared by dissolving 7.4\% Labrasol, 7.4\% Cremophor ${ }^{\circledR}$ EL and 22.2\% propylene glycol in distilled water (Table 2-1). Based on the results of pregabalin solubility study, only $40 \%$ of the predetermined pregabalin dose $(0.6 \%$, that was selected after a preliminary study) was dissolved in the internal aqueous phase of the primary $\mathrm{W} / \mathrm{O} \mathrm{ME}$ and the remaining $60 \%$ was dissolved in the external aqueous phase. The bioadhesive polymers; chitosan $(1.1 \%)$, sodium alginate $(0.4 \%)$ or Carbopol $981(0.15 \%)$ was soaked and allowed to swell overnight in the previously prepared external aqueous phase (1\% acetic acid was used instead of water in case of chitosan).

\section{Preparation of the final multiple W/O/W ME}

For preparation of the final formulation, the previously prepared drug-loaded primary W/O ME was incorporated accurately drop wise into the drug-loaded external aqueous polymeric solution (75) and gently mixed under magnetic stirring until a clear final eye drops was obtained. 
Table 2-1. Composition of pregabalin-loaded bioadhesive multiple $\mathrm{W} / \mathrm{O} / \mathrm{W} \mathrm{ME}$

\begin{tabular}{lccc}
\hline Ingredient (\%W/W) & Chitosan ME & Sod. alginate ME & Carbopol ME \\
\hline Pregabalin & 0.6 & 0.6 & 0.6 \\
Labrafac lipophile WL1349 & 7.8 & 7.8 & 7.8 \\
Capryol 90 & 6.5 & 6.5 & 6.5 \\
Lecithin & 6.5 & 6.5 & 6.5 \\
Labrasol & 7.4 & 7.4 & 7.4 \\
Cremophor ${ }^{\circledR}$ EL & 7.4 & 7.4 & 7.4 \\
Propylene glycol & 22.2 & 22.2 & 22.2 \\
Chitosan & 1.1 & - & - \\
Sod. alginate & - & 0.4 & - \\
Carbopol 981 & - & - & 0.15 \\
\hline
\end{tabular}




\section{Results}

\section{Our constructed ternary and pseudo-ternary phase diagrams}

For preparation of stable ME system, the HLB value of the surfactant used during the preparation of the W/O ME should be fall in the range of 3-6 while the HLB value of the surfactant that required for the $\mathrm{O} / \mathrm{W} \mathrm{ME}$ formation should be in the ange of 8-18 (76). In the current work, we followed this rule during the preparation of our multiple ME. For the preparation of the primary W/O ME we selected surfactant with low HLB value $(\leq 5)$ such as Capryol 90, lecithin and transcutol P. On the other hand, for preparation of the final W/O/W ME, all the used surfactants possessed high HLB values $(\geq 12)$ such as Labrasol, Tween 80, poloxamer 188, brij 97 and Cremophor ${ }^{\circledR}$ EL.

Figure 2-3 illustrated the different constructed ternary and pseudo-ternary phase diagrams with the $\mathrm{W} / \mathrm{O} \mathrm{ME}$ shown in the shaded regions. These regions represented all the possible combinations of the three ME components-aqueous phase, oil phase and surfactant or surfactants mixture - that were capable of forming a transparent primary $\mathrm{W} / \mathrm{O} \mathrm{ME}$. By selecting any point in that region, we determined the percentage of the three components that were easily mixed to form a W/O ME. Figure 2-3B was selected to pick up one point to prepare our $\mathrm{W} / \mathrm{O} \mathrm{ME}$ as it showed the largest shaded area so it gave us a higher number of points for selection. The selected W/O ME point consisted of $20 \%$ water $+30 \%$ oil (Labrafac lipophile WL1349) $+50 \%$ surfactants mixture (Capryol $90 \&$ soybean lecithin, 1:1). Two important criteria should be available in the point to be selected; the first is its ability to form a stable primary W/O ME. The second criterion is to be able to incorporate the highest possible amount of water as an internal phase in order to dissolve as much as possible of the required drug dose ( $0.6 \%$ pregabalin $)$.

It has been reported that the hydrocarbon chain length of the oil and the surfactant(s) is one of the most important factors that affects the ME region in the triphase diagram. Coming to the selected pseudo-ternary phase diagram (Figure 2-3B) in our study there was a correlation between the hydrocarbon chain length of - Labrafac oil \& Capryol 90 \& lecithin - and the large W/O ME region. Beginning with the two surfactants - Capryol 90 \& lecithin-Capryol 90 is a propylene glycol monoester of caprylic acid (C8). Such a medium hydrocarbon chain allowed it to be easily inserted between the two long hydrocarbon chains of lecithin in order to form a homogenous interfacial film at the interface between the Labrafac oil phase and the aqueous phase (72). Secondly, our oil phase (Labrafac lipophile WL 1349) is a triglyceride ester of caprylic $(\mathrm{C} 8)$ and capric $(\mathrm{C} 10)$ acids which possessed nearly the same carbon chain length of Capryol 90 that allow more homogenous mixing between our ingredients and better emulsification behavior.

Labrafac lipophile WL 1349, as a medium chain triglyceride esters has a higher solvent capacity and is less susceptible to oxidation compared to long chain triglycerides (77). Also, being an ester of saturated medium chain acids (C8 \& C10), our oil phase possessed a higher shelf stability compared to those containing unsaturated centers (78). 

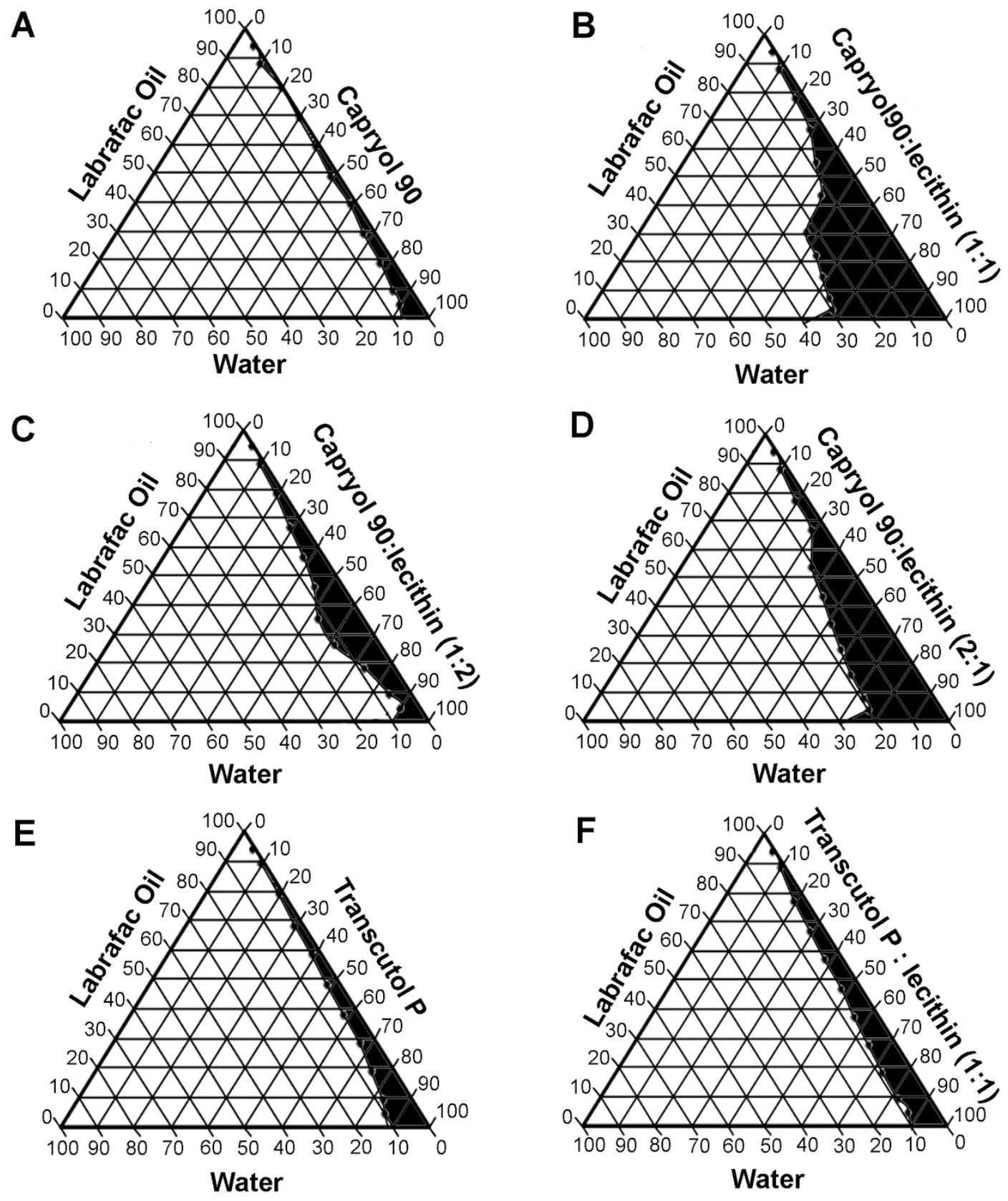

Figure 2-3. Ternary and pseudo-ternary phase diagrams of the primary W/O ME

The ME was formed of Labrafac oil/water and surfactant or surfactant mixture:

(A) Capryol 90, (B) Capryol 90: lecithin [1:1], (C) Capryol 90: lecithin [1:2], (D)

Capryol 90: lecithin [2:1], (E) Transcutol P and (F) Transcutol P: lecithin (1:1). The black colored regions represent the W/O ME area. Figure 2-3B was selected to pick up one point to prepare our W/O ME as it showed the largest ME shaded area which gave us a higher number of points for selection. 
Additionally, the shorter the hydrocarbon chain length of the oil the deeper the oil penetration into the hydrophobic tails of the surfactant allowing a higher stability of the interfacial film leading to the formation of the ME (65). In addition of being compatible with Capryol 90, lecithin was selected as a surfactant in our ME because it has the advantage of being safe, biocompatible, non-toxic, naturally occurring and well-tolerated surfactant $(47,72)$. Furthermore, it is in many respects regarded as an ideal biological surfactant because of its biodegradation (79). Despite of all these advantages lecithin possessed undesirable properties that may hinder its use as a surfactant for preparation of ME. In fact, it has two opposite characteristics as it has a strong hydrophobicity due to the presence of its two long hydrocarbon chains and in the same time it has strong lipophobicity due to the presence of hydrated zwitterionic polar groups and a strong tendency to form lamellar liquid crystalline phase. This limitation could be overcome by combining it with other surfactant in order to decrease its rigidity (72). Capryol 90 provided our ME system with this advantage and decreased the rigidity of lecithin by being incorporated into the interfacial film allowing the formation of ME.

\section{The external aqueous phase surfactant/co-surfactant mixture composition}

Among all the screened surfactants and co-surfactants, surfactant mixture of $7.4 \%$ Labrasol and 7.4\% Cremophor ${ }^{\circledR}$ EL in the presence of $22.2 \%$ propylene glycol as a cosurfactant (75) was the most efficient external aqueous phase to produce our final multiple W/O/W ME (Figure 2-4). This combination of surfactants and co-surfactant helped to incorporate the maximum amount of the primary W/O ME $(26 \% \mathrm{w} / \mathrm{w})$ during the final emulsification process in order to prepare our final multiple $\mathrm{W} / \mathrm{O} / \mathrm{W} \mathrm{ME}$.

Regarding the safety of our ME system, several studies previously reported the safety of the different surfactants and co-surfactant that have been used in the preparation of our formulations at the specific concentrations. Fialho et al used Cremophor ${ }^{\circledR}$ EL at a concentration of $15 \%$ in their ophthalmic ME formulation of dexamethasone to the eye (74). Also, they stated that according to the information provided by the manufacturer, Cremophor ${ }^{\circledR}$ EL at a concentration up to $30 \%$ does not have any irritant effect on the eye (80) (74). Furthermore, solutions of propylene glycol cause no irritations to the rabbit eye at a concentration up to $50 \%$, whereas the undiluted propylene glycol only associated with a weak conjunctival redness $(79,81,82)$. Also, Labrasol as a nonionic surfactant is comprised of mixture of mono-, di- and triglycerides and mono- and di-fatty acid esters of polyethyleneglycol, with caprylic and capric acids as the predominant fatty acids. Thus, Labrasol is compatible with both the oil phase (Labrafac lipophile) and the internal hydrophobic surfactant (Capryol 90).

On the other hand, Liu et al reported that Labrasol at a concentration of $5 \%$ could produce a slight ocular irritation when used as ophthalmic penetration enhancer for their drug (baicalin) (83). Despite of using Labrasol as a surfactant in our ME at a concentration above the limit that was previously reported, our results showed that it didn't produce any significant ocular irritation. Thus, the excellent ocular tolerability of our ME might be due to the demulcent effect of propylene glycol that capable of masking the potential irritant effect produced by Labrasol at the used concentration (84). 


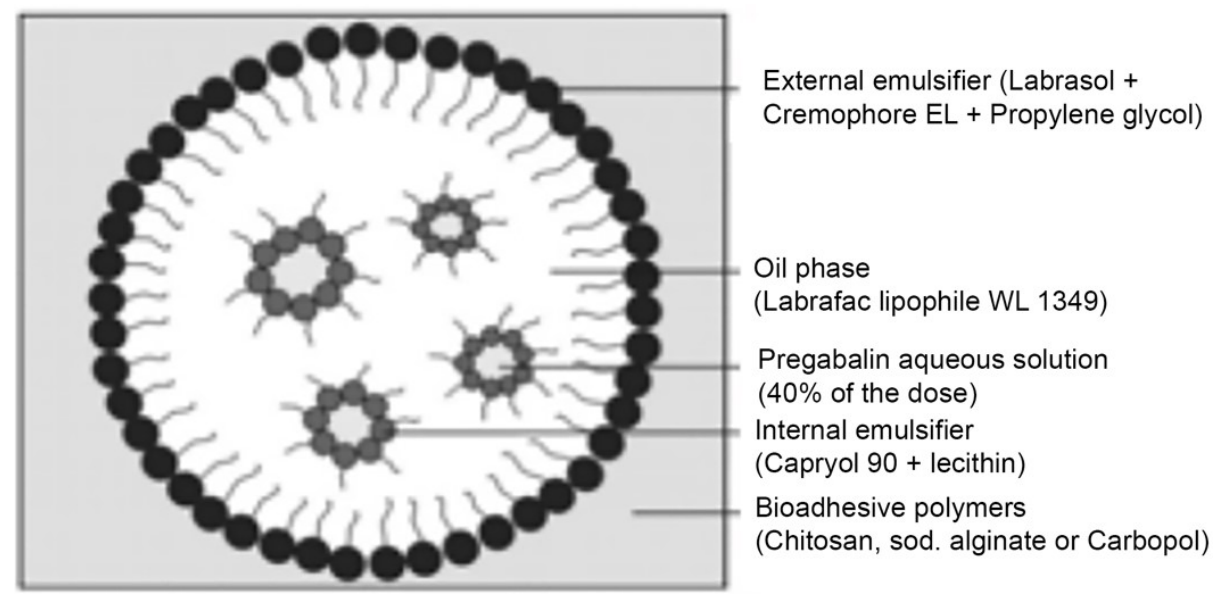

Figure 2-4. Cartoon showing composition of pregabalin-loaded multiple $\mathrm{W} / \mathrm{O} / \mathrm{W}$ ME eye drops

Our new developed multiple ME consists of Labrafac lipophile WL1349 as the oil phase, MilliQ water as the internal aqueous phase, mixture of Capryol 90 \& soybean lecithin, $1: 1$ as the hydrophobic surfactant/cosurfactant and mixture of Labrasol and Cremophor ${ }^{\circledR}$ EL in the presence of propylene glycol as the external hydrophilic surfactant/ cosurfactant. Hence, we have incorporated $40 \%$ of the predetermined pregabalin dose in the innermost aqueous phase of our developed ME system while incorporating the remaining dose $(60 \%)$ in the external polymeric aqueous phase to delay and sustain the drug release. 


\section{Pregabalin solubility}

Solubility studies showed that pregabalin solubility in water, 0.01N HCL and $0.01 \mathrm{~N} \mathrm{NaOH}$ was $37.7 \pm 0.16,41.48 \pm 0.30$ and $39.28 \pm 0.25 \mathrm{mg} / \mathrm{ml}$, respectively. In contrast the pregabalin concentration in Labrafac lipophile WL 1349 or the surfactant mixture (Capryol 90 \& soybean lecithin, 1:1 mixture) was under the detection limit. Water was selected to be used as internal aqueous phase because our preliminary study demonstrated that there was an incompatibility between the ME components and the acidic or alkaline internal aqueous phases. Based on the solubility data we dissolved $40 \%$ of the pregabalin dose in the internal aqueous phase while the remaining $60 \%$ was dissolved in the external aqueous phase. 


\section{CHAPTER 3. IN VITRO CHARACTERIZATION OF THE PREPARED PREGABALIN-LOADED W/O/W ME EYE DROPS}

\section{Introduction}

Ophthalmic drug delivery via simple solution eye drops are relatively inefficient because of the different clearance mechanisms in the eye. Actually, the drug residence time and the drug transport through the corneal epithelium are considered the main two factors that determine the drug bioavailability after topical instillation of eye drops. However, the optimum ocular delivery system should be applied once or twice per day without causing irritation or blurred vision and at the same time applied easily as eye drops. Currently, MEs are one of the most interesting ocular drug carrier systems that are designed to address multiple challenges in the ophthalmic delivery. MEs have been reported to achieve a remarkable increase in the residence contact time (85), an enhancement in the drug penetration (86) and a controlled sustained release of several ophthalmic drugs (50).

Because of the negatively charged corneal epithelium, MEs that include cationic surfactants showed an electrostatic interaction with the corneal epithelium that leads to an increase in the residence time, which would cause a higher drug concentration in the precorneal area and thus a greater improvement in the bioavailability. Also, an increase in the residence time of $\mathrm{ME}$ ophthalmic formulations could be attributed to the increase in the tear film viscosity after ME application. Furthermore, during the ME preparation, a polymer can be incorporated in the aqueous phase to increase the mucoadhesion and hence the residence time of the applied formulation. Some studies reported the effectiveness of the added bioadhesive polymer in controlling the release kinetics from ME eye drops.

In this chapter we described how our prepared multiple W/O/W ME formulations were evaluated for their droplet size, polydispersity index (PDI), zeta potential, morphology, viscosity, bioadhesion, in vitro drug release and corneal permeability. Furthermore, we performed immunohistochemistry to determine the expression of CACNA2D1-pregabalin target protein — in the Dutch belted rabbit eyes.

\section{Materials and Methods}

Sodium phosphate dibasic, sodium bicarbonate, potassium chloride, potassium dihydrogen phosphate, calcium chloride dihydrate, dipotassium hydrogen phosphate, glacial acetic acid, Triton X-100, Cremophore ${ }^{\circledR}$ EL, MTT reagent

(methylthiazolyldiphenyl- tetrazolium bromide) and glutathione disulfide were purchased from Sigma-Aldrich (St. Louis, MO). Propylene glycol, magnesium chloride hexahydrate, dextrose, Tween 80 , sodium chloride, gastric mucin (type II), dimethyl sulfoxide (DMSO) and phosphoric acid were procured from Fisher Scientific (Fair Lawn, NJ). Ethyl alcohol was purchased from Decon Labs, Inc. (King of Prussia, PA). 
Regenerated cellulose membrane (molecular weight cut-off 5,000 Da, Harvard Apparatus Co., Holliston, MA). Keratinocyte-SFM serum free medium was purchased from Life Technologies Corporation (Grand Island, NY). DMEM/F-12 (Dulbecco's modified Eagle's medium/nutrient mixture F-12) was purchased from Mediatech, Inc. (A Corning Subsidiary Manassas, VA). Fresh whole eyes of male New Zealand white rabbits were procured from Pel-Freez Biologicals (Rogers, AR).

\section{Measurement of the pH}

The $\mathrm{pH}$ values of each $\mathrm{ME}$ formulation were measured using a $\mathrm{pH}$ meter (Corning pH meter 440; Corning Inc., Corning, New York). One gram of each formulation was dispersed in $20 \mathrm{~mL}$ of MilliQ water then the $\mathrm{pH}$ was measured. The experiment was repeated three times and the results were presented as mean $\pm \mathrm{SD}$.

\section{Average droplet size, polydispersity index (PDI) and zeta potential measurement}

The mean droplet size, PDI and zeta potential (ZP) of our ME formulations were determined after suitable dilution using Zetasizer (Nanoseries, nano-ZS, Malvern Instruments Limited, UK) (86). All measurements were performed at $25 \pm 2{ }^{\circ} \mathrm{C}$. The results of three independent test runs were presented as mean $\pm \mathrm{SD}$.

\section{Transmission electron microscopy}

The morphology of our pregabalin-loaded ME eye drops was examined using transmission electron microscopy (TEM) (JEOL JEM1200EX II electron microscope). Briefly, the ME formulation was diluted 1:100 with deionized water. Two microliters of the diluted ME were placed on 400 mesh copper grids covered with formvar film (Electron Microscopy Sciences EMS, Hatfield, PA). The grids were allowed to dry overnight in a desiccator before examination by TEM.

\section{Determination of the viscosity}

A cone $\left(1.5^{\circ}\right)$ and plate rotary viscometer (Brookfield DV-II + programmable viscometer; Brookfield Engineering Laboratories, Middleboro, MA) was used to determine the viscosity of our ME disperse system according to our previously published protocol $(87,88)$. Each formulation $(500 \mu l)$ was placed on the stationary plate of the viscometer for $5 \mathrm{~min}$ before each measurement to reach the running temperature (89). The viscosity was measured in triplicate at $35 \pm 0.5^{\circ} \mathrm{C}$ and the results were calculated as mean \pm SD. 


\section{Measurement of the bioadhesive force}

The bioadhesive force of our ME eye drops was determined by a simple method that depends on evaluation of the rheological synergism that happen upon mixing the bioadhesive polymer with mucin dispersions $(90,91)$. Gastric mucin type II $(15 \%$, w/v) was dispersed in simulated tear fluid $(\mathrm{pH} 7.4)$ and allowed to dissolve overnight at $4^{\circ} \mathrm{C}$. Immediately before measurment, mucin dispersion was warmed to $35^{\circ} \mathrm{C}$ and then mixed with our prepared ME formulations that had been warmed to the same temperature. Then, the viscosities of mucin dispersion, formulations and their mixture were measured in triplicate using the Brookfield viscometer. Thus, viscosity change due to bioadhesion as well as the bioadhesive forces were calculated employing the following equations

(Equation 3-1 and Equation 3-2) and the results were represented as mean $\pm \mathrm{SD}(90,91)$.

$$
\begin{aligned}
& \eta_{b}=\eta_{t}-\left(\eta_{m}+\eta_{p}\right) \\
& F_{b}=\eta_{b} \times \gamma
\end{aligned}
$$

where $\eta_{b}$ is the viscosity due to bioadhesion, $\eta_{t}$ is the viscosity of the mixture, $\eta_{m}$ is the viscosity of mucin dispersion, $\eta_{p}$ is the viscosity of the formulations, $F_{b}$ is the bioadhesive force and $\gamma$ is the shear rate at which the viscosity value was measured.

\section{In vitro drug release}

Pregabalin release behavior from our ME eye drops was studied according to our previously published protocol $(89,92)$. Briefly, we used $1500 \mu$ fast micro-equilibrium dialyzers to which a semipermeable regenerated cellulose membrane with molecular weight cut-off 5,000 Da was attached (Harvard Apparatus Co., Holliston, MA). One hundred microliters of our ME formulations or the controls were placed in the donor chamber. The used control formulations were $0.6 \%$ simple pregabalin aqueous solution or $0.6 \%$ pregabalin viscous solution using different polymers (chitosan, sodium alginate and Carbopol 981). Warmed phosphate buffer $\mathrm{pH} 7.4\left(1.5 \mathrm{ml}\right.$ at $\left.35 \pm 0.5^{\circ} \mathrm{C}\right)$ was placed in the receptor chamber. The dialyzer was kept in thermostatically controlled shaker (at 50 rpm and $35 \pm 0.5^{\circ} \mathrm{C}$ ). At predetermined time intervals ranging from 0.25 to $24 \mathrm{~h}$, the entire medium in the receptor chamber was withdrawn and replaced immediately by $1.5 \mathrm{ml}$ of fresh warmed buffer solution. Sink conditions were maintained at all times. All experiments were carried out in triplicate and the concentration of the released drug was then determined by HPLC. The cumulative percent amount released of pregabalin was calculated as mean $\pm \mathrm{SD}$.

\section{Corneal permeability study}

Modified rounded junction Franz diffusion cells (PermeGear Inc) were used to measure the pregabalin corneal permeability. Corneas were mounted to the cells with the epithelial side facing the donor chamber containing the ME formulations (93). 
A circulating water bath was used to maintain the temperature of the cells at a $35 \pm 0.5^{\circ} \mathrm{C}$ $(11,94)$. One hundred microliters of pregabalin-loaded ME eye drops - chitosan ME, sodium alginate $\mathrm{ME}$ and Carbopol $981 \mathrm{ME}$, in addition to the two controls, pregabalin aqueous solution and pregabalin-loaded in Carbopol 981 viscous solution-was placed in the donor chamber. The receptor chamber was continuously stirred and was filled with 5 $\mathrm{mL}$ of balanced salt solution enriched with bicarbonate, dextrose, and glutathione (BSSPLUS $^{\circledR}$, Alcon Laboratories Inc., Fort Worth, TX) (11). At predetermined time intervals $(1,2,3,4,5$ and $6 \mathrm{~h}), 500 \mu \mathrm{L}$ were withdrawn from the receptor chamber and replaced with an equal volume of fresh warmed BSS-PLUS ${ }^{\circledR}$. The drug concentration in withdrawn samples was immediately determined by HPLC assay as described above. The results were plotted as cumulative amount permeated $(\mu \mathrm{g})$ versus time. Also, the steadystate flux (SSF) was calculated by dividing the rate of transport at steady state by the surface area of the cornea through which permeation occurred.

Flux ( $\mathrm{J})$ as well as permeability coefficient (P) was calculated using the following equations (Equation 3-3 and Equation 3-4) (93, 95):

$$
\begin{aligned}
& \text { Flux }(\mathrm{J})=(\mathrm{dM} / \mathrm{dt}) / \mathrm{A} \\
& \text { Permeability }(\mathrm{P})=\text { Flux } / \mathrm{C}_{d}
\end{aligned}
$$

where, $\mathrm{M}$ is the cumulative amount of drug transported, $\mathrm{A}$ is the surface area of the corneal membrane $\left(0.636 \mathrm{~cm}^{2}\right)$ exposed to the drug and $\mathrm{C}_{\mathrm{d}}$ is the initial drug concentration in the donor chamber.

The data were statistically analyzed using one-way analysis of variance (ANOVA) test followed by Tukey's multiple comparisons test. Statistical calculations were carried out using GraphPad Prism 7 software (GraphPad Software Inc., San Diego, CA).

\section{Immunohistochemistry (IHC)}

To immunolocalize pregabalin target protein - CACNA2D1 - in Dutch belted rabbits' paraffin-embedded eye sections (50 $\mu \mathrm{m}$ thickness), we used our previously published methods $(96,97)$. Briefly, we used $10 \%$ goat serum to block non-specific binding sites in tissue sections. An anti-CACNA2D1 monoclonal antibody (ThermoFisher Scientific, Rockford,USA, catalog \# MA3-921; 1:100) was used in our study as the primary antibody along with donkey anti-mouse Alexa fluor ${ }^{\circledR} 488$ secondary antibody (Invitrogen, Waltham, MA; catalog \#A21202; 1:200) and DAPI (Vector Laboratories, Inc., CA; catalog \#H-1200) as a nuclear counterstain. Vector ${ }^{\circledR}$ TrueVIEW ${ }^{\mathrm{TM}}$ Autofluorescence Quenching Kit (Vector Laboratories, Inc., CA; catalog \#SP-8400) was used to remove the unwanted background autofluorescence in our sections. Sections were viewed and images were obtained using a Nikon C1 confocal microscope (Nikon, NY). 


\section{Results}

\section{Average droplet size, PDI and zeta potential}

Table 3-1 listed the apparent mean droplet size, PDI and ZP values of the oil phase of both blank and medicated diluted ME formulations. The droplet size of all the prepared ME formulations either blank or medicated were $<20 \mathrm{~nm}$ with PDI values $<$ 0.38 . This minuscule droplet size is very important to ensure high bioavailability. Because it greatly helped the transcorneal penetration of our ME formulations as it is well known that a droplet size less than $200 \mathrm{~nm}$ is required for accepted passive drug targeting through biological membranes $(98,99)$. The obtained small droplet size of our ME was an expected outcome because of the presence of propylene glycol as a co-surfactant. As this co-surfactant could penetrate the interfacial film formed by the surfactants mixture at the oil/water interface, it lowered its viscosity and made it more flexible which in turn resulted in the formation of micro-droplets that have a smaller-radius curvature, which finally resulted in the production of transparent $\mathrm{ME}$ systems $(74,100)$. In addition, Nidhin et al., 2008 reported that PDI values ranges from 0.01 to 0.5 is an indication for a narrow size distribution while values higher than 0.7 indicate a very broad distribution (101). Similarly, our prepared ME formulations had a narrow droplet size distribution $(\mathrm{PDI}<0.38)$.

Concerning the zeta potential, both blank and medicated sodium alginate and Carbopol MEs possessed large negative charges $(>-26 \mathrm{mV})$. On the other hand, both blank and medicated chitosan MEs were positively charged. This difference in the type of the charge is an inherent property for the used polymer. Whatever its type, the surface charge of any colloidal system is very important factor to achieve a long-term stability as it resulted in repulsion between droplets and prevented their coalescence upon standing.

\section{TEM}

The TEM image revealed that the microstructure of our ME appeared as a homogenously distributed separate single ME droplets with a spherical regular outline (Figure 3-1). Also, the results of TEM supported the droplet size data obtained by the Zetasizer.

\section{Viscosity and bioadhesion}

Figure 3-2. presents the viscosity and bioadhesion force values of the prepared formulations containing $0.6 \%$ pregabalin. The tested formulations included; $\mathrm{ME}$ formulations prepared using three different bioadhesive polymers - chitosan, sodium alginate and Carbopol 981 - compared to their control formulations that prepared from the same polymers at the same concentrations in absence of the ME colloidal system. Formulations contained Carbopol 981 either in the presence or absence of ME system 
Table 3-1. Droplet size, PDI and zeta potential of blank and medicated ME formulations

\begin{tabular}{|c|c|c|c|c|c|c|}
\hline \multirow[b]{2}{*}{ Type of ME eye drops } & \multicolumn{2}{|c|}{ Mean droplet size (nm) } & \multicolumn{2}{|c|}{ PDI } & \multicolumn{2}{|c|}{ Zeta potential $(\mathrm{mV})$} \\
\hline & Blank & Medicated & Blank & Medicated & Blank & Medicated \\
\hline PRG chitosan ME & $17.4 \pm 0.0$ & $17.4 \pm 0.3$ & $0.36 \pm 0.0$ & $0.37 \pm 0.0$ & $15.9 \pm 3.3$ & $10.2 \pm 2.3$ \\
\hline PRG sod. alginate ME & $16.8 \pm 0.3$ & $16.5 \pm 0.2$ & $0.34 \pm 0.0$ & $0.34 \pm 0.0$ & $-26.8 \pm 0.8$ & $-26.7 \pm 1.7$ \\
\hline PRG Carbopol $981 \mathrm{ME}$ & $16.0 \pm 0.2$ & $15.4 \pm 0.1$ & $0.26 \pm 0.0$ & $0.26 \pm 0.0$ & $-30.1 \pm 2.2$ & $-26.3 \pm 1.0$ \\
\hline
\end{tabular}




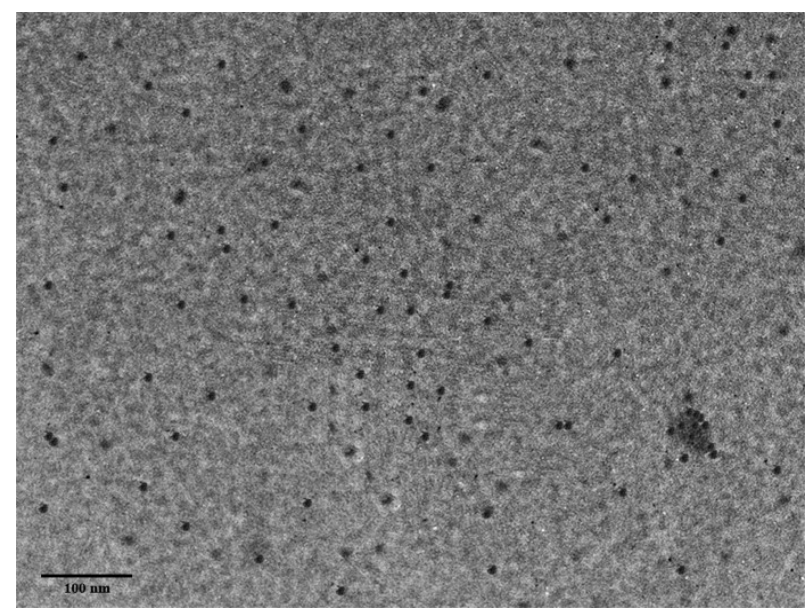

\section{Figure 3-1. TEM image of pregabalin-loaded ME}

Our ME appeared as a homogenously distributed separate single ME droplets with a spherical regular outline with a small droplet size $(<100 \mathrm{~nm})$.
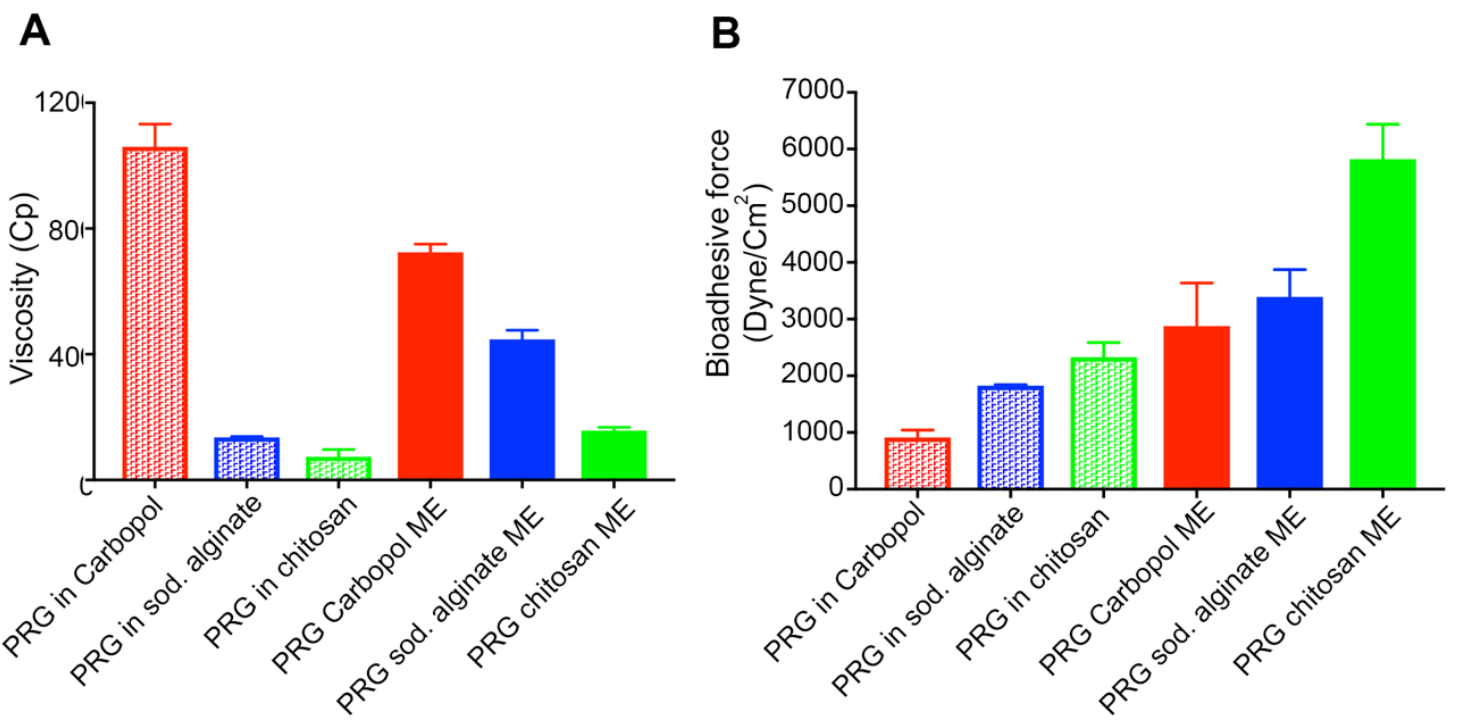

Figure 3-2. Viscosity and bioadhesion of pregabalin-loaded ME formulations and controls

The viscosity values in $\mathrm{cP}(\mathrm{A})$ and the bioadhesive force in dyne $/ \mathrm{cm}^{2}(\mathrm{~B})$ of pregabalinloaded ME eye drops as well as the controls were calculated as mean $\pm \mathrm{SD} ; \mathrm{n}=3$. Formulations contained Carbopol 981 either in the presence or absence of ME system have the highest viscosity values compared to those contained chitosan or sodium alginate. However, chitosan-including formulations possessed the highest bioadhesion force compared to the formulations that included the other polymers. 
have the highest viscosity values compared to those contained chitosan or sodium alginate (Figure 3-2A). This difference in the formulation viscosity may be attributed to the difference in the concentration, crosslinking and molecular weight of the used polymers (102). Regarding the bioadhesion of our formulations, chitosan-including formulations possessed the highest bioadhesion force compared to the formulations that included the other polymers. Actually, there are several factors that may affect the bioadhesive force of the polymer including; the ability of the polymer to swell, the extent of swelling, the polymer chain flexibility and the type of binding with mucin (103). Thus, this high bioadhesive force of chitosan might be attributed to its ability to interact with mucin via three different types of interaction- $-\mathrm{H}$-bonding, hydrophobic association and electrostatic binding due to its carried positive charge - which allowed it to strongly bind to mucin compared to sodium alginate and Carbopol which bound only with $\mathrm{H}$ - bonding (104). Also, the used chitosan possessed high chain flexibility due to its moderate degree of acetylation (15-25\%), which allowed it to adequately interact with mucin and resulted in a higher bioadhesive force $(105,106)$. Meanwhile Figure 3-2B showed that our pregabalin ME formulations have significantly higher bioadhesive force compared to the corresponding control formulations $(\mathrm{p}<0.0001, \mathrm{p}<0.01$ and $\mathrm{p}<0.05$ for chitosan, Carbopol and sodium alginate respectively). The improved bioadhesion of our ME formulations might be attributed to the presence of non-ionic surfactants which improved the polymers' chains wetting and swelling and promoted their interaction with mucin which resulted in better bioadhesion (103). These results indicated that our ME pregabalin eye drops would be expected to have a longer corneal contact time and longer duration of action after application into the eye.

\section{In vitro release}

In vitro release profiles of pregabalin-loaded ME formulations as well as their control formulations - pregabalin aqueous solution and pregabalin-loaded in different bioadhesive polymers viscous solutions; chitosan, sodium alginate or Carbopol 981 —are shown in Figure 3-3. Despite being water-soluble drug that characterized by a fast release behavior, our ME formulations succeeded to slow and sustain the release of pregabalin up to $24 \mathrm{~h}$ in contrast to the control formulations that exhibited rapid release of all the drug loaded in the first few hours.

Figure 3-3 illustrated that all ME formulations showed an initial fast release $(\approx 25 \%$ in the first $0.5 \mathrm{~h})$ followed by a controlled release for the next $24 \mathrm{~h}$. Actually, this release behavior was expected and it was considered as a normal outcome for the special engineering of our multiple $\mathrm{W} / \mathrm{O} / \mathrm{W} \mathrm{ME}$ formulation. Being a multiple $\mathrm{ME}$ where small pregabalin-loaded water droplets were entrapped within larger oil droplets that in turn were dispersed into an external pregabalin-loaded aqueous phase. Thus, our ME unique structure could govern the drug distribution inside the formulation. Only $40 \%$ of the drug dose was located in the innermost layer of the ME while the remaining 60\% was incorporated in the external polymeric outermost aqueous layer. However, the initial fast release might be due to the presence of this $60 \%$ of the drug dose in the external aqueous phase, which was ready to be released once being in contact with the release medium 


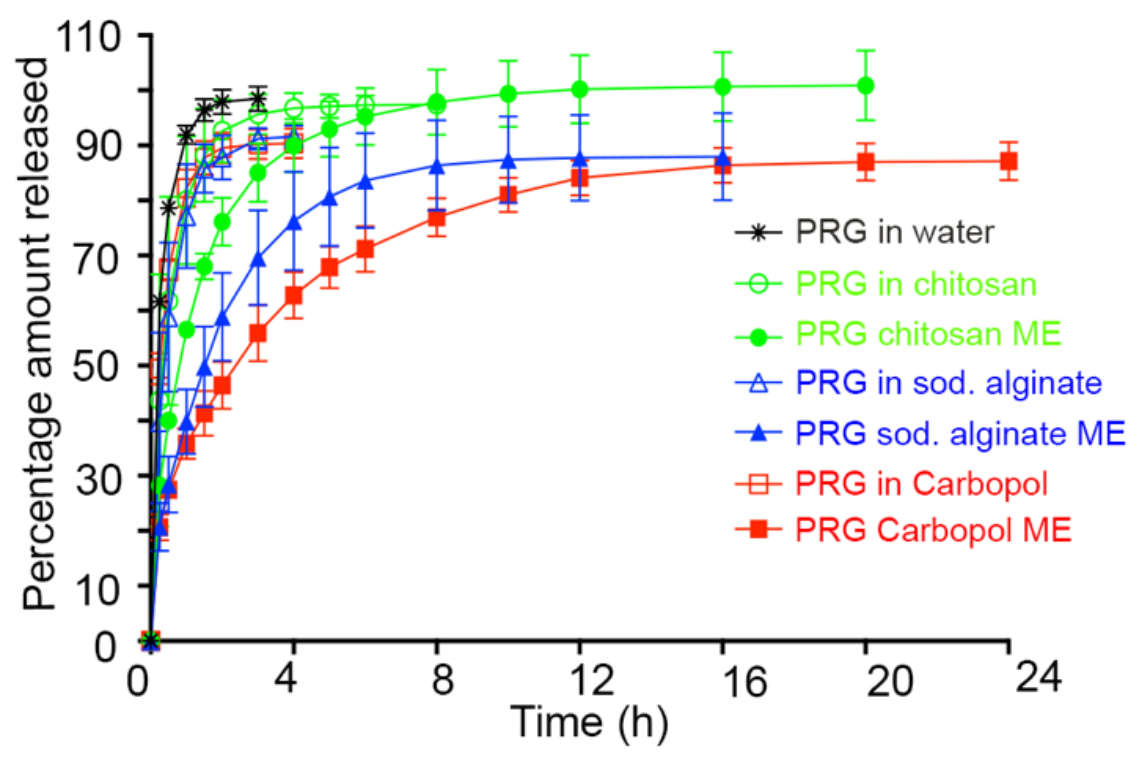

Figure 3-3. Cumulative amount released (\%) of pregabalin from ME eye drops

Data are expressed as mean $\pm \mathrm{SD} ; \mathrm{n}=3$.

Our ME formulations succeeded to slow and sustain the release of pregabalin up to $24 \mathrm{~h}$ in contrast to the control formulations that exhibited rapid release of all the drug loaded in the first few hours. 
after simple diffusion through the viscous polymeric external aqueous layer. On the other hand, the presence of $40 \%$ of the drug dose in the innermost aqueous layer. of the $\mathrm{ME}$ was responsible for the controlled release behavior that lasted up to $24 \mathrm{~h}$. The drug was located in the innermost aqueous layer, which was surrounded by an intermediate layer of oil. Therefore to be released, pregabalin must pass through two interfaces - the inner $\mathrm{W} / \mathrm{O}$ interface and the outer $\mathrm{O} / \mathrm{W}$ interface-after which it has to diffuse through the viscous bioadhesive polymer in the external aqueous layer. Also, since our drug is watersoluble and its solubility in oil is negligible, the presence of the oil layer might control its release and greatly prolong the time required for it. Because of this structure, the time required for the drug to pass through all these stages was greatly prolonged.

One more reason for this slow release was the presence of the drug in both innermost and outermost layers of our ME system. The presence of the drug in the external layer might hinder or slow the drug release from the innermost layer by affecting the drug concentration gradient across the intermediate oil layer of our ME and resulted in an overall slow release behavior (107). Moreover, the bioadhesive polymer ensured that the formulation would remain on the corneal surface for enough time to allow the sustained release of the drug. Finally, we could conclude that this specially designed release behavior was intended in order to produce a rapid onset with a longer duration of action upon instillation into the eye.

Comparing the rate of pregabalin release from the ME formulations and their controls, we found that all formulations could be arranged in the order of; pregabalin in water $>$ pregabalin in polymeric viscous solution $>$ pregabalin ME. This arrangement may be due to the free water solubility of pregabalin. When the drug being in aqueous solution, no more steps were required to be ready for corneal transport while one more diffusion step was added when being in viscous polymeric solution. On the other hand, several steps were required as previously mentioned when being in ME form.

The drug release profiles from these systems indicated two different release mechanisms at different stage of release: a large initial burst release followed by a slow release in the long time. The burst drug release was controlled by diffusion through the viscous polymeric external aqueous layer while the sustained time release was controlled by diffusion across the surfactant-covered interfacial film of the ME droplets.

\section{Corneal permeability}

In order to determine the drug transcorneal permeability from our ME formulations as well as from the two controls - pregabalin aqueous solution and pregabalin viscous polymeric solution - we used fresh rabbit corneas excised from the whole eyes of New Zealand white rabbits that shipped overnight from Pel-Freez Biologicals in Hanks balanced salt solution over wet ice. In order to keep the cornea alive during the whole experiment (6h) we used BSS-PLUS ${ }^{\circledR}$ irrigating solution as a receptor medium. This solution has a similar composition to aqueous humor and commonly used as a sterile intraocular irrigating solution due to its ability to maintain the anatomic and 
physiologic integrity of intraocular tissues $(108,109)$. As previously mentioned, pregabalin is a BCS class I drug, which suffers from a rapid absorption that leads to short duration of action. Actually, this process was against the required sustained release behavior. Therefore the availability of an appropriate drug delivery system that could maintain the high drug permeability and prolongs its corneal contact time is an urgent need. In the current study we succeeded to achieve this goal by incorporating pregabalin in our ME formulations.

Data shown in Table 3-2 demonstrated that our ME formulation could maintain the high permeability associated with pregabalin with no significant difference in the permeation rates (dM/dt) Figure 3-4., flux values (J) Figure 3-5. and permeability coefficients $(\mathrm{P})$ compared to the used controls (Overall $\mathrm{P}=0.4191)$. Furthermore, these results presented that the addition of a bioadhesive polymer (Carbopol 981) into an aqueous solution of pregabalin had no significant effect on the drug permeability. Similarly, Chen et al., 2007 reported that the addition of $0.75 \%$ carbomer 940 had no significant effect on the permeation rate of triptolide from ME (75). In addition to maintaining the high permeability of the drug, our ME formulations were capable of prolonging its corneal contact time and sustaining its release that resulted in improving its overall bioavailability. These results confirmed the previously obtained in vitro release results that showed the sustained release behavior of our formulations that lasted for up to $24 \mathrm{~h}$.

\section{Immunohistochemistry}

Immunohistochemistry was performed to determine if pregabalin-target protein, CACNA2D1 is expressed in eye tissues of Dutch belted rabbits that are associated with IOP modulation before testing the in vivo efficacy of our pregabalin-loaded eye drops in these animals. Figure 3-6. illustrated that CACNA2D1 was highly expressed in both the ciliary body (non-pigmented epithelium) and the trabecular meshwork where the aqueous humor production and drainge occurs respectively. Thus the mechanism through which pregabalin decrease the IOP might be through decreasing the aqueous humor production by the ciliary body or increasing its drainge through the trabecular meshwork or by both mechanisms. Interestingly the IHC results of Dutch belted rabbits are similar to what we got before in IHC of both mouse and human donor eyes. 
Table 3-2. In vitro transcorneal permeability parameters of pregabalin from multiple $\mathrm{W} / \mathrm{O} / \mathrm{W} \mathrm{ME}$ and control

\begin{tabular}{|c|c|c|c|}
\hline Formulation & $\begin{array}{c}\text { Rate of } \\
\text { permeation } \\
(\mathbf{d M} / \mathbf{d t})\end{array}$ & $\begin{array}{c}\text { Flux } \\
\left(\mu \mathrm{g} / \mathbf{c m}^{2} / \mathbf{m i n}\right)\end{array}$ & $\begin{array}{c}\text { Permeability } \\
\text { coefficient }(\mathbf{P}) \times 10^{-4} \\
(\mathrm{~cm} / \mathrm{s})\end{array}$ \\
\hline PRG in water & $0.285 \pm 0.1$ & $0.45 \pm 0.13$ & $7.5 \pm 2.2$ \\
\hline PRG in Carbopol & $0.374 \pm 0.15$ & $0.59 \pm 0.24$ & $9.8 \pm 3.9$ \\
\hline PRG chitosan ME & $0.233 \pm 0.06$ & $0.37 \pm 0.10$ & $6.1 \pm 1.7$ \\
\hline PRG sod. alginate ME & $0.287 \pm 0.18$ & $0.45 \pm 0.28$ & $7.5 \pm 4.7$ \\
\hline PRG Carbopol ME & $0.288 \pm 0.08$ & $0.45 \pm 0.13$ & $7.6 \pm 2.2$ \\
\hline
\end{tabular}

Data are expressed as mean $\pm \mathrm{SD} ; \mathrm{n}=6$

Overall $\mathrm{P}$ value $=0.4191$

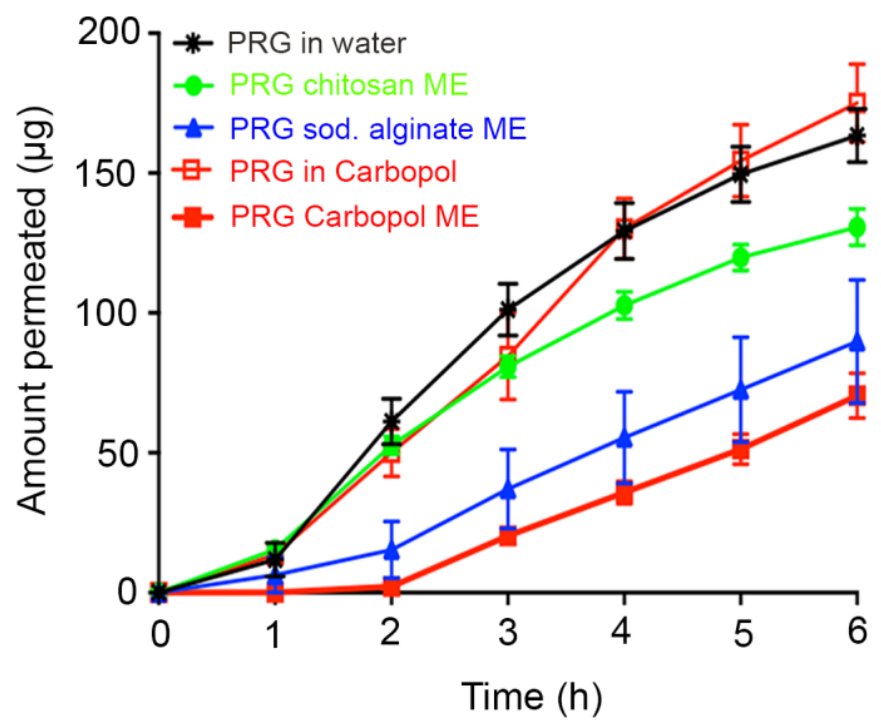

Figure 3-4. Cumulative amount permeated of pregabalin from ME eye drops using rabbit cornea

Data are expressed as mean $\pm \mathrm{SD} ; \mathrm{n}=6$

There was no significant difference (Overall $\mathrm{P}$ value $=0.4191$ ) between the cumulative amount permeated from our ME formulations and both the aqueous pregabalin solution and the viscous polymeric Carbopol solution. 


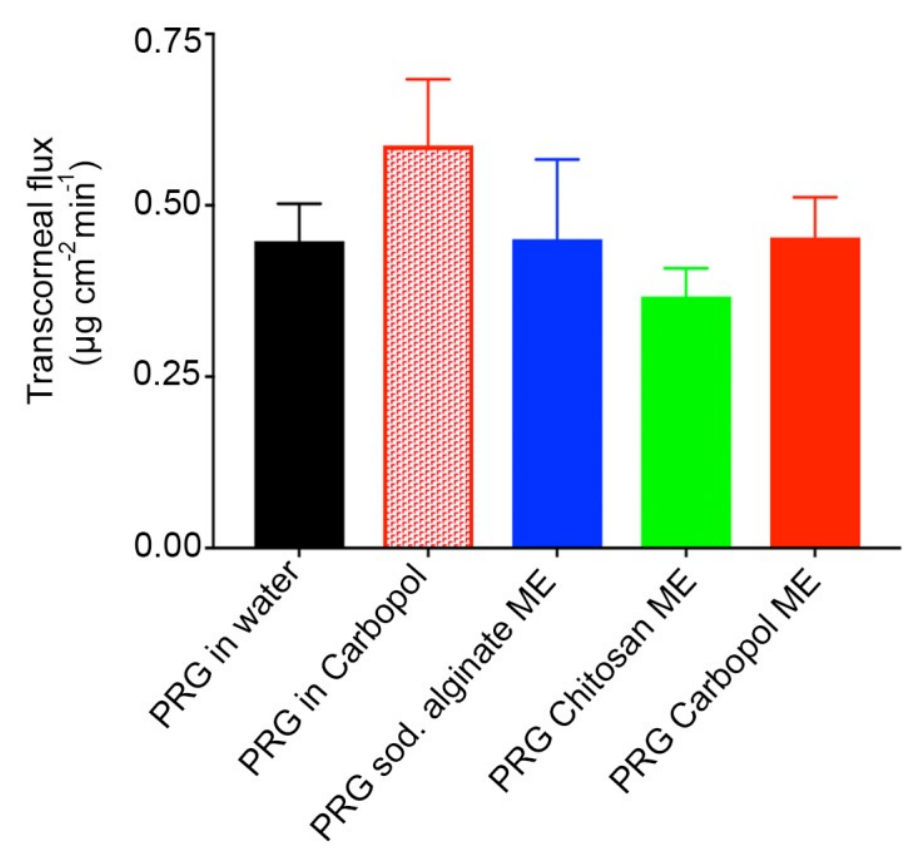

Figure 3-5. Transcorneal flux of pregabalin from pregabalin-loaded ME eye drops

Data are expressed as mean $\pm \mathrm{SD} ; \mathrm{n}=6$

There was no significant difference (overall $\mathrm{p}=0.4191$ ) between the transcorneal flux of pregabalin from our developed ME formulations and the drug flux from a simple aqueous solution. 

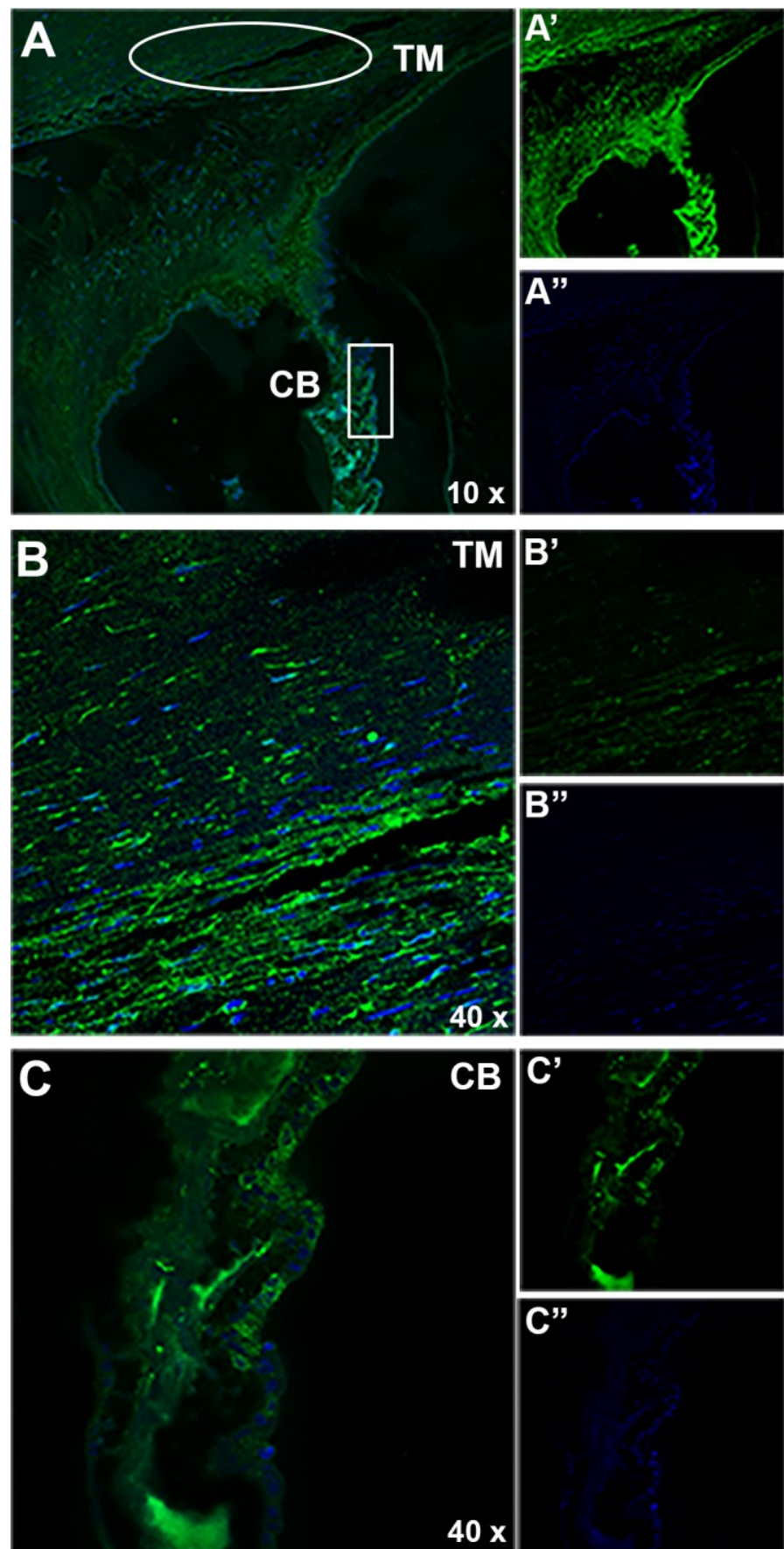

$40 \times$
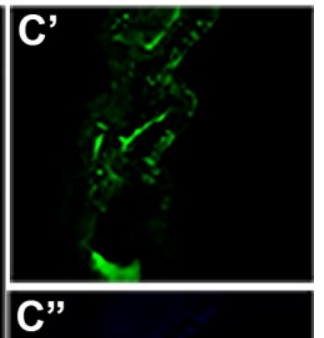

Figure 3-6. Cellular localization of CACNA2D1 in Dutch belted rabbits eye

A-C" sections from Dutch belted rabbits iridocorneal angle were labeled with antiCACNA2D1 antibodies. Pregabalin target protein-CACNA2D1 (green) - is localized in the trabecular meshwork (B) and the ciliary body (C). Blue nuclei, TM trabecular meshwork, CB ciliary body. 


\section{CHAPTER 4. DETERMINATION OF SAFETY, IN VIVO EFFICACY AND THE CELL TOXICITY OF THE MICROEMULSION EYE DROPS}

Our previously published data show that pregabalin hydroxypropylmethylcelluse (HPMC) viscous eye drops could maintain the IOP-lowering ability of the drug for up to $6 \mathrm{~h}$ without causing any irritation either for mice or Dutch belted rabbits eyes (Figure 4-1) (24). In contrast, we measured a sustained in vitro release behavior of pregabalin from our multiple $\mathrm{W} / \mathrm{O} / \mathrm{W}$ microemulsion eye drops that lasts for $24 \mathrm{~h}$. Because we will use the same drug in a different formulation, we want to make sure that our formulations ingredients are safe to a corneal epithelial cell line and will not harm the eye of the experimental animal. These data together suggest that our formulation will be safe to a corneal epithelial cell line. Also, it will demonstrate an IOP-lowering ability of the drug that may last for more than $24 \mathrm{~h}$. To determine the IOP-lowering ability and the mildness of our formulations, we will use Dutch belted rabbits.

The aim of the work in this chapter is to determine the safety and the efficacy (time and extent) of our developed ME eye drops after a single dose application in Dutch belted rabbits. Also, to measure the in vitro cytotoxicity of our formulations by the MTT assay using a human corneal limbal epithelial cell line (HCLE).

\section{Methods}

\section{Animals}

Dutch belted male rabbits $(2-2.5 \mathrm{Kg})$ procured from Robinson Services (Mocksville, NC) were used to test the IOP-lowering effects of our pregabalin-loaded ME formulations. All procedures including rabbits were approved by the Animal Care and Use review board of the University of Tennessee Health Science Center (UTHSC) and followed the Association of Research in Vision and Ophthalmology (ARVO) Statement for the Use of Animals in Ophthalmic and Vision Research in addition to the guidelines for laboratory animal experiments (Institute of Laboratory Animal Resources, Public Health Service Policy on Humane Care and Use of Laboratory Animals).

\section{In vivo evaluation of our formulation safety}

The safety of our developed multiple W/O/W ME eye drops was tested by installation of $100 \mu 1$ of the medicated formulation in the lower conjunctival sac of the right eye of Dutch belted rabbits $(n=3)$ while the left eye served as a control. Eyes were

examined every hour for any sign of irritation such as redness, tearing and conjunctival or corneal swelling. Slit-lamp examination was performed for all rabbits eyes at the end of the experiment (110). 

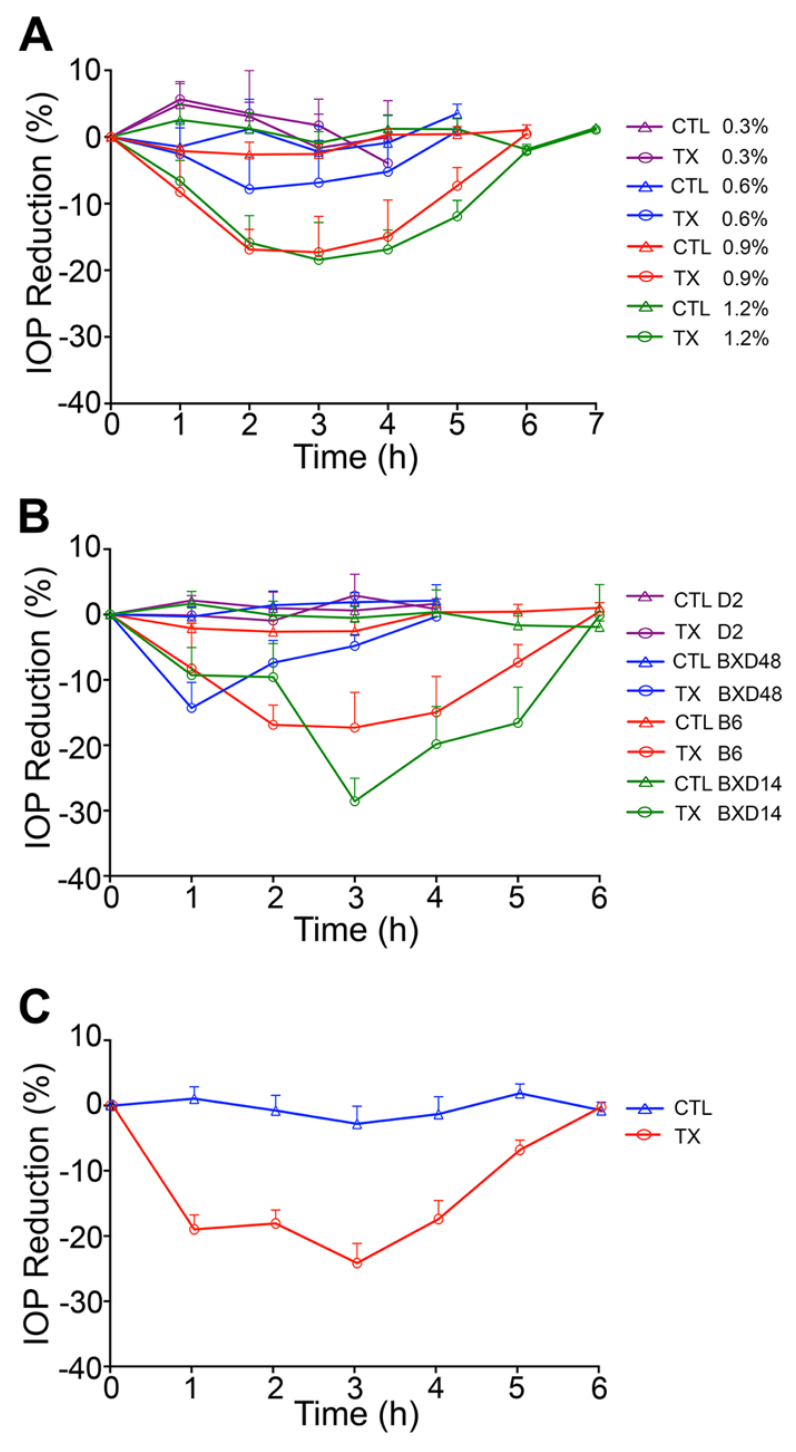

Figure 4-1. IOP reduction (\%) after application of pregabalin simple eye drops

Pregabalin, a specific modulator of CACNA2D1, lowered IOP in B6 mice in a dosedependent manner (A). We measured a dose-dependent reduction in IOP compared to control treatment after a single application of ophthalmic formulation containing a range of concentrations of pregabalin from 0.3 to $1.2 \%$. The minimal concentration required to produce the maximum reduction in IOP was $0.9 \%$ (mean $\pm \mathrm{SEM} ; \mathrm{n}=6)$. Pregabalininduced IOP reduction is haplotype-specific. Mice carrying the B parental allele of Cacna2d1 (i.e., B6 and BXD14) are more responsive to pregabalin $(0.9 \%)$ than mice carrying the D parental allele (i.e., D2 and BXD48; mean \pm SEM; $n=4-6$ ). A single dose of pregabalin eye drops $(0.9 \%)$ lowers IOP by $22.1 \%$ in Dutch belted rabbits $(n=5)(C)$. Reprinted with permission. Chintalapudi SR, Maria D, Di Wang X, Bailey JNC, consortium N, International Glaucoma Genetics c, et al. Systems genetics identifies a role for Cacna2d1 regulation in elevated intraocular pressure and glaucoma susceptibility. Nat Commun. 2017;8(1):1755. doi: 10.1038/s41467-017-00837-5. 


\section{In vivo evaluation of our formulation efficacy}

The IOP-lowering effect of our multiple ME formulations was determined using Dutch belted rabbits $(n=3)$ (111). During the study one hundred microliters of the ME eye drops were applied into the inferior conjunctival sac of the right eye of the Dutch belted rabbits while the left eye served as a control by receiving $100 \mu l$ of the blank ME formulation. The IOP was measured using a Tono-pen AVIA (Reichert Technologies, Depew, NY) immediately before the application of the formulation (baseline) and at predetermined time intervals until it returned back to its baseline value $(24,112)$. Five consecutive IOP readings were averaged for each individual eye at each measurement.

Evaluation of pregabalin formulations was based on comparing the calculated pharmacodynamic parameters including; maximum percent reduction in IOP [IOP reduction $(\%)$ ], the time required to reach maximum decrease in IOP $\left(\mathrm{T}_{\max }\right)$, the time required for IOP to return again to its baseline (i.e., end of drug effect; $T_{\text {end }}$ ) and the total area under the IOP reduction (\%)-versus-time curve $\left(\mathrm{AUC}_{\text {total }}\right)$. All results were expressed as mean \pm SEM.

\section{In vitro evaluation of formulation cell toxicity}

In vitro cytotoxicity of our pregabalin-loaded Carbopol ME eye drops (this formulation was selected based on the obtained in vivo results) was evaluated by the methylthiazolyldiphenyl tetrazolium bromide assay using a modified previously published protocol $(113,114)$. MTT assay is based on the ability of healthy cells (mitochondria) to reduce the MTT reagent into purple formazan crystals(115).The viability assay was carried out in 96-well plates (Costar 3596, Corning Inc., Corning, NY). Human corneal limbal epithelial cells (HCLE) (116)was seeded at a concentration of 18,000 cell/well and incubated in a humidified environment at $37^{\circ} \mathrm{C}$ in $5 \% \mathrm{CO} 2$ for $24 \mathrm{~h}$ in Gibco-Keratinocyte-SFM medium (1X) (supplemented with bovine pituitary extract, epidermal growth factor and $\mathrm{CaCl} 2.2 \mathrm{H} 2 \mathrm{O}$ ). After $24 \mathrm{~h}$, the medium was replaced by $50 \mu \mathrm{l}$ of artificial tears, then $150 \mu \mathrm{l}$ of the diluted formulation $(2.15 \mu \mathrm{l}$ formulation $+147.85 \mu 1$ medium) were added slowly above the artificial tears. This dilution was based on the surface area of the cell layer in the well exposed to the formulation compared to the actual rabbit corneal surface area. In addition, after different time points of incubation (1,2,3 and $6 \mathrm{~h}$ ), the formulation was removed and the plate was washed with the culture medium to remove all the traces of the formulation. Two hundred microliters of MTT reagent ( $1 \mathrm{mg} / \mathrm{ml}$ in culture medium) was added to each well and the plates were incubated at $37^{\circ} \mathrm{C}$ for $4 \mathrm{~h}$. After incubation the MTT was removed and $200 \mu \mathrm{L}$ DMSO was added to each well to dissolve the formazan crystals. The plate was then shaken for $15 \mathrm{~min}$. The absorbance of the solution was measured at $570 \mathrm{~nm}$ and converted to percent cell viability relative to the control (untreated cells) by a $\mu$-Quant universal microplate spectrophotometer (Bio-Tek Instruments, Inc. Winooski, VT). Statistical analysis of the percent cell viability data was performed using a one-way ANOVA followed by Tukey's multiple comparisons test. Each experiment was performed in eight replicates and the results expresed as mean \pm SEM. 


\section{Results}

\section{In vivo safety and efficacy}

Our recently published data showed that pregabalin $(0.9 \%$ in $2 \%$ HPMC viscous eye drops) has the ability to decrease IOP in both mice (28.6 $33.5 \%)$ and Dutch belted rabbits (22.1 $\pm 2.8 \%)$ while the IOP returned back to baseline after only 6h (Figure 4-1) (24). Thus, based on these results we sought to determine if our new sustained release glaucoma eye drops could maintain the IOP at lower level for longer period of time after a single drop application using Dutch belted rabbits. Figure 4-2 illustrated the percent IOP reduction after application of $0.6 \%$ pregabalin ME eye drops and Table 4-1 listed all the calculated pharmacodynamic parameters.

Among all the tested ME formulations, pregabalin Carbopol ME provided the maximum IOP-lowering effect $(42.3 \pm 2.6 \%$ reduction in IOP) that returned to baseline at $32.7 \pm 1.3 \mathrm{~h}$ after application (AUC $=169.9 \pm 13.4 \mathrm{mmHg} . \mathrm{h}$ ) compared to the control formulation (pregabalin in Carbopol in absence of ME), which produced only a $29.4 \pm 1.4 \%$ IOP reduction that returned to baseline at $9.3 \pm 0.7 \mathrm{~h}$ (AUC=39 $\pm 4 \mathrm{mmHg} . \mathrm{h})$. Regardless the type of polymer, all our prepared ME formulations induced an IOP \% reduction $>34 \%$ (Table $4-1)$.

Statistical analysis of different pharmacodynamic parameters (Table 4-2) showed that there was no significant difference either in percent IOP reduction or $\mathrm{T}_{\max }$ values between all the tested formulation including control ( $\mathrm{P}=0.2504$ and 0.9567 , respectively). This non-significant difference between all the tested formulations might be due to the presence of free drug that was available to produce a rapid onset that reached its maximum after $3 \mathrm{~h}$. On the other hand, the $\mathrm{T}_{\text {end }}$ values of our ME eye drops were significantly longer compared to the control formulation $(\mathrm{p}<0.0001)$. This might be due to the ability of our ME formulations to sustain pregabalin release and prolong its corneal contract time.

The IOP-lowering results of our ME formulations proved that we have succeeded to achieve our goal to produce bioadhesive formulations with rapid onset and long duration of action. The main cause of the rapid onset was the presence of $60 \%$ of the drug dose in the external aqueous phase of the ME while the presence of the remaining $40 \%$ in the innermost aqueous layer was responsible for the long duration of action that lasted for $32.7 \pm 1.3 \mathrm{~h}$ for the Carbopol ME. Upon comparing the IOP-lowering effects of the three tested ME, it was found that they could be arranged as follow; Carbopol ME > sodium alginate $\mathrm{ME}>$ chitosan ME. The main cause of this arrangement might be the difference in their viscosities (Figure 3-2A). The high viscosity of Carbopol ME and its in situ gelling ability at the physiological $\mathrm{pH}$ (117) prevented its drainage outside the eye and allowed enough time for the bioadhesion interaction to occur. In addition, the slow release rate together with the high viscosity and bioadhesion allowed for the longer duration of action. Although chitosan ME has the highest value of the bioadhesive force (Figure 3-2B), it has the lowest IOP-lowering 


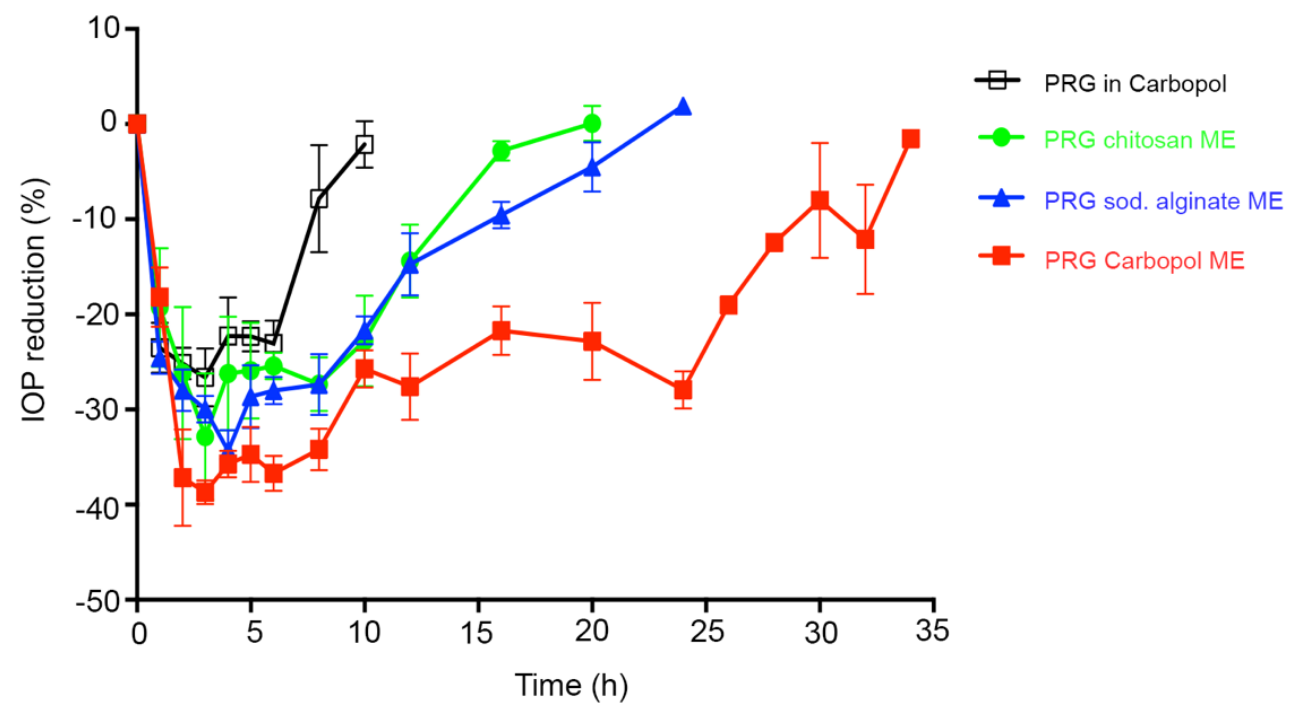

Figure 4-2. IOP reduction (\%) after $0.6 \%$ pregabalin ME eye drops application in Dutch belted rabbits

Data are expressed as mean $\pm \mathrm{SD} ; \mathrm{n}=3$.

Pregabalin-loaded Carbopol ME eye drops provided the maximum IOP-lowering effect $(42.3 \pm 2.6 \%$ reduction in IOP) that returned to baseline at $32.7 \pm 1.3 \mathrm{~h}$ after application (AUC $=169.9 \pm 13.4 \mathrm{mmHg} . \mathrm{h}$ ) compared to the control formulation (pregabalin in Carbopol in absence of ME system), which produced only a $29.4 \pm 1.4 \%$ IOP reduction that returned to baseline at $9.3 \pm 0.7 \mathrm{~h}(\mathrm{AUC}=39 \pm 4 \mathrm{mmHg} . \mathrm{h})$. 
Table 4-1. Pharmacodynamic parameters after application of $0.6 \%$ pregabalinloaded ME chitosan, sod. alginate, Carbopol 981 and control eye drops to Dutch belted rabbits

\begin{tabular}{lcccc}
\hline Pharmacodynamic & \multicolumn{4}{c}{ Ophthalmic eye drops } \\
\cline { 2 - 5 } parameters & $\begin{array}{c}\text { PRG } \\
\text { chitosan } \\
\text { ME }\end{array}$ & $\begin{array}{c}\text { PRG sod. } \\
\text { alginate ME }\end{array}$ & $\begin{array}{c}\text { PRG } \\
\text { Carbopol } \\
\text { ME }\end{array}$ & $\begin{array}{c}\text { PRG in } \\
\text { Carbopol }\end{array}$ \\
\hline Baseline IOP & $21.2 \pm 0.4$ & $20.9 \pm 0.2$ & $21.4 \pm 0.7$ & $22.1 \pm 0.4$ \\
IOP at T max & $13.9 \pm 1.0$ & $13.6 \pm 0.2$ & $12.3 \pm 0.5$ & $15.7 \pm 0.4$ \\
$\Delta$ IOP & $-7.3 \pm 1.3$ & $-7.3 \pm 0.4$ & $-9.1 \pm 0.8$ & $-6.5 \pm 0.4$ \\
\% Reduction in IOP & $34.1 \pm 5.4$ & $35.0 \pm 1.7$ & $42.3 \pm 2.6$ & $29.4 \pm 1.4$ \\
T $_{\text {max }(h)}$ & $4.0 \pm 1.0$ & $3.7 \pm 0.3$ & $3.3 \pm 0.9$ & $3.3 \pm 1.5$ \\
Tend $_{\text {(h) }}$ & $20.0 \pm 0.0$ & $24.0 \pm 0.0$ & $32.7 \pm 1.3$ & $9.3 \pm 0.7$ \\
AUC total $_{\text {(mmHg. h) }}$ & $69.5 \pm 14.2$ & $80.7 \pm 4.6$ & $169.9 \pm 13.4$ & $39.0 \pm 4.0$ \\
\hline
\end{tabular}

Data are expressed as mean \pm SEM; $\mathrm{n}=3$

$\mathrm{T}_{\max }(\mathrm{h})$, time to maximum response in hours;

$\mathrm{T}_{\text {end }}(\mathrm{h})$, time to end of response in hours;

$\mathrm{AUC}_{\text {total }}(\mathrm{mmHg} . \mathrm{h})$, total area under IOP versus time curve 
Table 4-2. Statistical comparisons among different pregabalin ME eye drops and the control after application of a single dose

\begin{tabular}{lccccccc}
\hline $\begin{array}{l}\text { Pharmacodynamic } \\
\text { Parameters }\end{array}$ & $\begin{array}{c}\text { Overall } \\
\text { P value }\end{array}$ & $\begin{array}{c}\text { Carbopol. vs } \\
\text { Sod. alginate }\end{array}$ & $\begin{array}{c}\text { Carbopol. vs } \\
\text { Chitosan }\end{array}$ & $\begin{array}{c}\text { Carbopol. vs } \\
\text { CTL }\end{array}$ & $\begin{array}{c}\text { Sod. alginate } \\
\text { vs Chitosan }\end{array}$ & $\begin{array}{c}\text { Sod. alginate } \\
\text { vs CTL }\end{array}$ & $\begin{array}{c}\text { Chitosan vs } \\
\text { CTL }\end{array}$ \\
\hline \% Reduction in IOP & 0.2504 & $>0.05$ & $>0.05$ & $>0.05$ & $>0.05$ & $>0.05$ & $>0.05$ \\
Tmax $($ h) & 0.9567 & $>0.05$ & $>0.05$ & $>0.05$ & $>0.05$ & $>0.05$ \\
Tend (h) & $<0.0001$ & $<0.001$ & $<0.0001$ & $<0.0001$ & $<0.05$ & $<0.0001$ \\
AUC total (\%. h) & 0.0001 & $<0.01$ & $<0.001$ & $<0.0001$ & $>0.05$ & $>0.05$ & $>0.0001$ \\
\hline
\end{tabular}

CTL: control

Overall $p$ value represents the outcome of the one-way ANOVA analysis.

Individual $p$ values represent the outcome of Tukey-Kramer multiple comparisons tests. 
capacity among our tested $\mathrm{ME}$ formulations ( $\mathrm{T}_{\text {end }}$ and $\left.\mathrm{AUC}_{\text {total }}\right)$ (Table 4-1). This might be due to its low viscosity that led to the drainage of some of the formulation outside the eye before being strongly adhered to the eye surface.

Although both pregabalin-loaded Carbopol ME and the control formulation contained Carbopol 981 as a polymer at the same concentration, the ME possessed better IOP-lowering capacity and longer duration of action (Figure 4-2 and Table 41). This might be due to the higher bioadhesive force of the $\mathrm{ME}$ formulation due to its content of non-ionic surfactants that have the ability to improve the polymer chain wetting and swelling and so promoted its interaction with the mucin layer that covered the eye surface and so resulted in stronger bioadhesion and longer duration of action (103).

Similarly, Naveh et al., (118) reported that emulsion-based pilocarpine eye drops might be used as a once-daily therapy for glaucoma compared to several daily applications of generic pilocarpine simple eye drops. However, they presented that a single dose of pilocarpine emulsion eye drops induced a great reduction in IOP of normotensive rabbits compared to the generic eye drops $(28.5 \%$ and $18 \%$, respectively), they encouraged further studies to be done to determine the mechanism through which emulsion eye drops produced this marked reduction of IOP with a long duration of action.

Regarding the safety of our ME eye drops, the slit-lamp biomicroscopic examination that performed after the IOP of Dutch belted rabbits returned back to baseline in the IOP in vivo study showed that there was no sign of irritation such as redness or swelling. In addition, cornea was clear with normal smooth epithelial surface, no flare in aqueous humor and lens was clear (Figure 4-3).

\section{In vitro cytotoxicity}

The results of in vitro cytotoxicity assay of our pregabalin-loaded Carbopol ME and both negative and positive controls at different time points (1,2,3 and $6 \mathrm{~h}$ ) are illustrated in Figure 4-4. The obtained results showed that the cell viability of our tested formulation was significantly different from that of the positive control ( $1 \%$ Triton-X $100)$ at all the time points $(\mathrm{p}<0.0001)$. Upon comparing the cell viability of the tested formulation with that of the negative control (untreated cells), it was found that there was no significant difference at all the different tested time points $(\mathrm{p}>0.9)$, which indicated that pregabalin as a drug and both Carbopol and our multiple ME system have no cytotoxicity to HCLE cell line. Furthermore, the time of incubation of our formulation with the HCLE cells was a very important parameter during the cytotoxicity assay. Because we thought to consider the strong bioadhesion and the long contact time of our ME formulation, we determined the cell viability after 1, 2, 3 and $6 \mathrm{~h}$ of incubation. Similarly, Andres-Guerrero et al., (116) studied the cytotoxicity of their new formulation of Timolol maleate that contained bioadhesive polymers as well as the traditional Timolol maleate eye drops using HCLE cell cultures after 1 and $4 \mathrm{~h}$ of incubation. They reported 


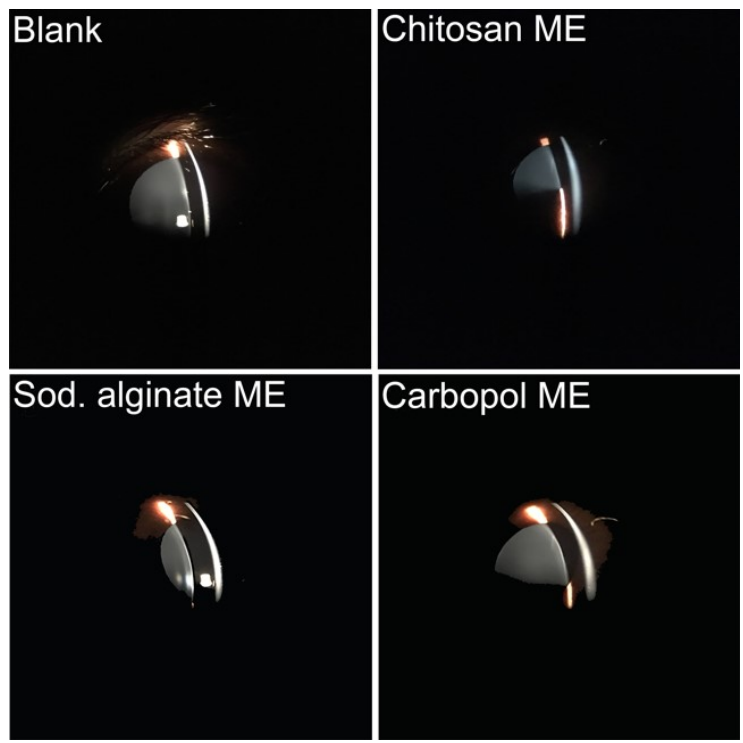

Figure 4-3. Slit lamp examination after application of pregabalin-loaded ME eye drops

Cornea and lens were clear with no signs of irritation after application of a single dose of our developed ME eye drops - chitosan ME, sodium alginate ME and Carbopol ME — in Dutch belted rabbits. 


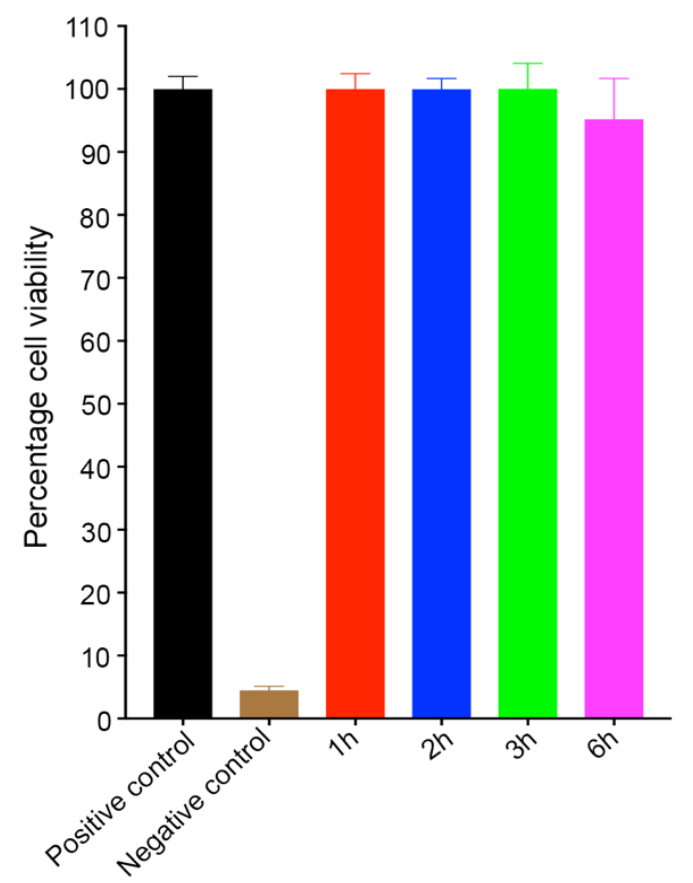

Figure 4-4. Cytotoxicity histogram of pregabalin-loaded Carbopol ME eye drops using HCLE

Data are expressed as mean $\pm \mathrm{SD} ; \mathrm{n}=8$.

Pregabalin as a drug and both Carbopol and our multiple ME system have no cytotoxicity to HCLE cell line at the different studied incubation periods (1,2,3 and $6 \mathrm{~h})$. 
that as their formulation had a bioadhesive polymers that would increase the contact time of their formulations on the eye surface, so to stimulate a long-term therapy they did the assay for longer period of time (1 and 4 hours).

However, 15 minutes of incubation is sufficient for testing the cell toxicity of conventional topical ocular formulations which supposed to be eliminated from the eye surface within only 5 minutes (116). 


\section{CHAPTER 5. STABILITY STUDY OF PREGABALIN-LOADED CARBOPOL 981 ME EYE DROPS}

\section{Introduction}

The main purpose of the stability testing of a pharmaceutical drug product is to establish its shelf life and the different recommended storage conditions. Hence, the drug product should be exposed to a variety of environmental conditions for a specific time including temperature, humidity and light. To be able to study the stability of a new drug substance or a new drug product the International Conference on Harmonisation of technical requirements for registration of pharmaceuticals for human use (ICH) stated a specific guidance that defined the stability data package required for a registration application in the United States, the European Union and Japan. According to the ICH guidelines the new tested drug product should be packaged in a container closure system that is the same or imitates the actual packaging for storage and distribution. Also, at least three batches of the pharmaceutical product are required to conduct the stability study (119).

For long-term stability study the testing frequency should be at least every three months during the first year, every six months for the second year then annually. However, a minimum of three time points including the initial and the final time points is required for a six-month accelerated stability study. If a significant change in the measured parameters observed during the stability study, adding more testing time points is highly recommended (119).

Based on the results that we got from our in vivo experiments, among all the tested pregabalin ME eye drops; pregabalin-loaded Carbopol $981 \mathrm{ME}$ formulation was selected for conducting a variety of stability study tests.

The aim of the work in this chapter was to investigate the physical and the chemical stability of our prepared pregabalin-loaded Carbopol $981 \mathrm{ME}$ eye drops. Longterm stability study was conducted at two temperatures $-5^{\circ} \mathrm{C} \pm 3^{\circ} \mathrm{C}$ and $25^{\circ} \mathrm{C} \pm 2^{\circ} \mathrm{C}-$ for a period of four months. Nevertheless, accelerated stability study was conducted at $40^{\circ} \mathrm{C}$ $\pm 2^{\circ} \mathrm{C}$ while intermediate stability study was conducted at $30^{\circ} \mathrm{C} \pm 2^{\circ} \mathrm{C}(75)$ for a period of four months. During the stability study the measured parameters were including; $\mathrm{pH}$, drug content, droplet size, polydispersity index (PDI), zeta potential as well as the in vitro release of our prepared ophthalmic formulation. To study the stability of our pregabalinloaded Carbopol $981 \mathrm{ME}$ eye drops, three batches were prepared exactly by the same procedure mentioned before in chapter 2 . 


\section{Physical Stability}

\section{Freeze-thaw cycles}

Three samples from the prepared three batches of pregabalin-loaded Carbopol 981 ME eye drops were submitted to freeze-thaw cycles $\left(-20\right.$ to $\left.25^{\circ} \mathrm{C}\right)$ of $48 \mathrm{~h}$ for three cycles and assessed for their physical stability by evaluating their appearance.

\section{Centrifugation test}

We also thought to investigate the physical stability of our ME disperse system by the centrifuge test(75). Three samples from three different batches of our prepared ophthalmic formulation were submitted to centrifugation at different forces of $40.10 \mathrm{~g}$, $80.20 \mathrm{~g}, 160.40 \mathrm{~g}, 320.80$ and 481.20 (5.000, 10.000, 20.000, 40.000, 50.000 and 60.000 $\mathrm{rpm}$ ) for $30 \mathrm{~min}$ using laboratory centrifuge (SORVALL, WX Ultra Series Centrifuge, USA) and assessed for phase separation, drug precipitation, creaming and droplet aggregation (120).

\section{Chemical Stability}

To study the chemical stability of our ME eye drops we thought to follow the guidelines established by the ICH. These guidelines stated that if the drug product is intended to be stored in a refrigerator the long-term stability study should be assessed at $5^{\circ} \mathrm{C} \pm 3^{\circ} \mathrm{C}$ for 12 months while the accelerated stability study should be conducted at $25^{\circ} \mathrm{C} \pm 2{ }^{\circ} \mathrm{C}$ for also 12 months. In addition, the ICH guidelines stated that there is a general case that should be applied for the drug product storage conditions when there is no specific temperature established for storage.

In the general case the long-term stability study should be conducted at $25^{\circ} \mathrm{C} \pm$ $2^{\circ} \mathrm{C}$ while the accelerated stability study should be conducted at $40^{\circ} \mathrm{C} \pm 2{ }^{\circ} \mathrm{C}$. Also, in the general case there is an intermediate stability study that should be conducted at $30^{\circ} \mathrm{C} \pm$ $2^{\circ} \mathrm{C}$.

In our stability study our prepared pregabalin-loaded ME eye drops were filled in a white dropper bottles under aseptic conditions then stored in thermostatically controlled air ovens at $25^{\circ} \mathrm{C} \pm 2{ }^{\circ} \mathrm{C}(121), 30^{\circ} \mathrm{C} \pm 2{ }^{\circ} \mathrm{C}$ and $40^{\circ} \mathrm{C} \pm 2{ }^{\circ} \mathrm{C}$ and ambient humidity for four months. Also, our formulations were stored in a refrigerator at $5^{\circ} \mathrm{C} \pm 3^{\circ} \mathrm{C}$ for four months. In addition, to easily monitor any change in the physical appearance of our $\mathrm{ME}$ eye drops, our formulation was filled in a transparent glass bottles $(7 \mathrm{ml})$ at each studied temperature. The tested formulation was evaluated initially and at specified time intervals (1, 2, 3 and 4 months) for the following parameters: 


\section{pH of the prepared multiple ME formulation}

Five hundred milligrams of our ophthalmic formulation were dispersed in $10 \mathrm{ml}$ of deionized water, then, the $\mathrm{pH}$ value was determined using $\mathrm{pH}$ meter.

\section{Drug content of the prepared multiple ME formulation}

To determine the drug content of our ME formulation one hundred milligrams was accurately weighed in a stoppered volumetric $10 \mathrm{ml}$ measuring flask. Three milliliters of deionized water were added and flask was shaken for 10 mins, then $7 \mathrm{ml}$ of absolute ethyl alcohol was added and flask was shaken for additional 10 mins, then brought to volume $(10 \mathrm{ml})$ with ethanol and the contents of the flask were mixed well. The solution was filtered using $0.22 \mu \mathrm{m}$ membrane filters (Millipore, Billerica MA) and assayed for the total pregabalin content by HPLC, using the method described in chapter 2. The drug content determination was carried out in triplicate.

\section{Average droplet size, PDI and zeta potential}

To measure the mean droplet size, PDI and zeta potential of our ME eye drops Zetasizer was used after suitable dilution of our formulation as previously mentioned in chapter 3 . The results of the three batches at each temperature were presented as mean \pm SD.

\section{In vitro release}

To determine if the sustained release pattern of pregabalin from our developed multiple $\mathrm{W} / \mathrm{O} / \mathrm{W}$ microemulsion eye drops changes with time under the different storage temperatures, the in vitro release was performed initially and at different time points $(1,2,3$ and 4 months) under the different storage temperatures, we used $1500 \mu 1$ fast microequilibrium dialyzers and followed exactly the same procedure in chapter 3.

The data were statistically analyzed using one-way analysis of variance (ANOVA) test followed by Dunnett's multiple comparisons test. Statistical calculations were carried out using GraphPad Prism 7 software (GraphPad Software Inc., San Diego, CA). 


\section{Results}

\section{Physical stability}

Our prepared pregabalin-loaded Carbopol $981 \mathrm{ME}$ disperse system submitted to both freeze-thaw cycles and centrifugation were observed to be physically stable without any sign of creaming, turbidity, phase separation or precipitation. Also, in a period of 4 months of the stability study at $5^{\circ} \mathrm{C}, 25^{\circ} \mathrm{C}, 30^{\circ} \mathrm{C}$ and $40^{\circ} \mathrm{C}$, no signs of physical instability were observed except a slight yellow color of the formulation at $40^{\circ} \mathrm{C}$ after four months which confirmed the high stability of our developed ME eye drops specifically at $5^{\circ} \mathrm{C}, 25^{\circ} \mathrm{C}, 30^{\circ} \mathrm{C}$.

\section{pH of our multiple ME eye drops}

Our developed pregabalin-loaded Carbopol $981 \mathrm{ME}$ eye drops had a $\mathrm{pH}$ value of $5.32 \pm 0.21$ at the beginning of the stability study while reached a maximum value of $5.43 \pm 0.15$ at $5^{\circ} \mathrm{C}$ and a minimum value of $4.84 \pm 0.07$ at $40^{\circ} \mathrm{C}$ after four months. Figure 5-1 and Table 5-1 presented the obtained results at the different time points of the stability study $(1,2,3$ and 4 months $)$ at the four different studied temperatures $\left(5^{\circ} \mathrm{C}, 25^{\circ} \mathrm{C}\right.$, $30^{\circ} \mathrm{C}$ and $40^{\circ} \mathrm{C}$ ). There was no significant change on the $\mathrm{pH}$ value during the test period (four month) except at $40^{\circ} \mathrm{C}(\mathrm{p}<0.01)$ (Table 5-2). The obtained results of $\mathrm{pH}$ values confirmed the high stability of our ME eye drops even at high temperatures $\left(30^{\circ} \mathrm{C}\right.$ and $\left.40^{\circ} \mathrm{C}\right)$.

\section{Average droplet size, polydispersity index (PDI) and zeta potential}

Figure 5-2 and Table 5-3 showed the results of the mean droplet size at different time points of the stability tests at four different temperatures. The initial average droplet size of our ME eye drops was $15.58 \pm 0.09$ while the highest measured value of the droplet size after four months of stability study was $17.14 \pm 0.82$. After one month, there was no significant alteration in the measured droplet size ( $p>0.05)$ (Table 5-4). However, there was a significant change after two months $(\mathrm{p}<0.05)$ at only $40^{\circ} \mathrm{C}$ and after three months at both $30^{\circ} \mathrm{C}(\mathrm{p}<0.05)$ and $40^{\circ} \mathrm{C}(\mathrm{p}<0.01)$. In addition, after four month of stability study there was no significant change in the average droplet size at both $25^{\circ} \mathrm{C}$ and $30^{\circ} \mathrm{C}$ $(\mathrm{p}>0.05)$ while there was a significant change at both $5^{\circ} \mathrm{C}(\mathrm{p}<0.05)$ and $40^{\circ} \mathrm{C}(\mathrm{p}<0.05)$. Overall our ME eye drops have a high stability because it maintained its small droplet size after a four-month of stability study at all the different studied temperatures. Also, it has been reported that $\mathrm{ME}$ with smaller droplet size are considered to be more stable than those with larger droplets (59).

Figure 5-3 and Table 5-5 presented the results of the PDI during the stability tests. After four months of stability study the highest value of PDI of our ME eye drops 


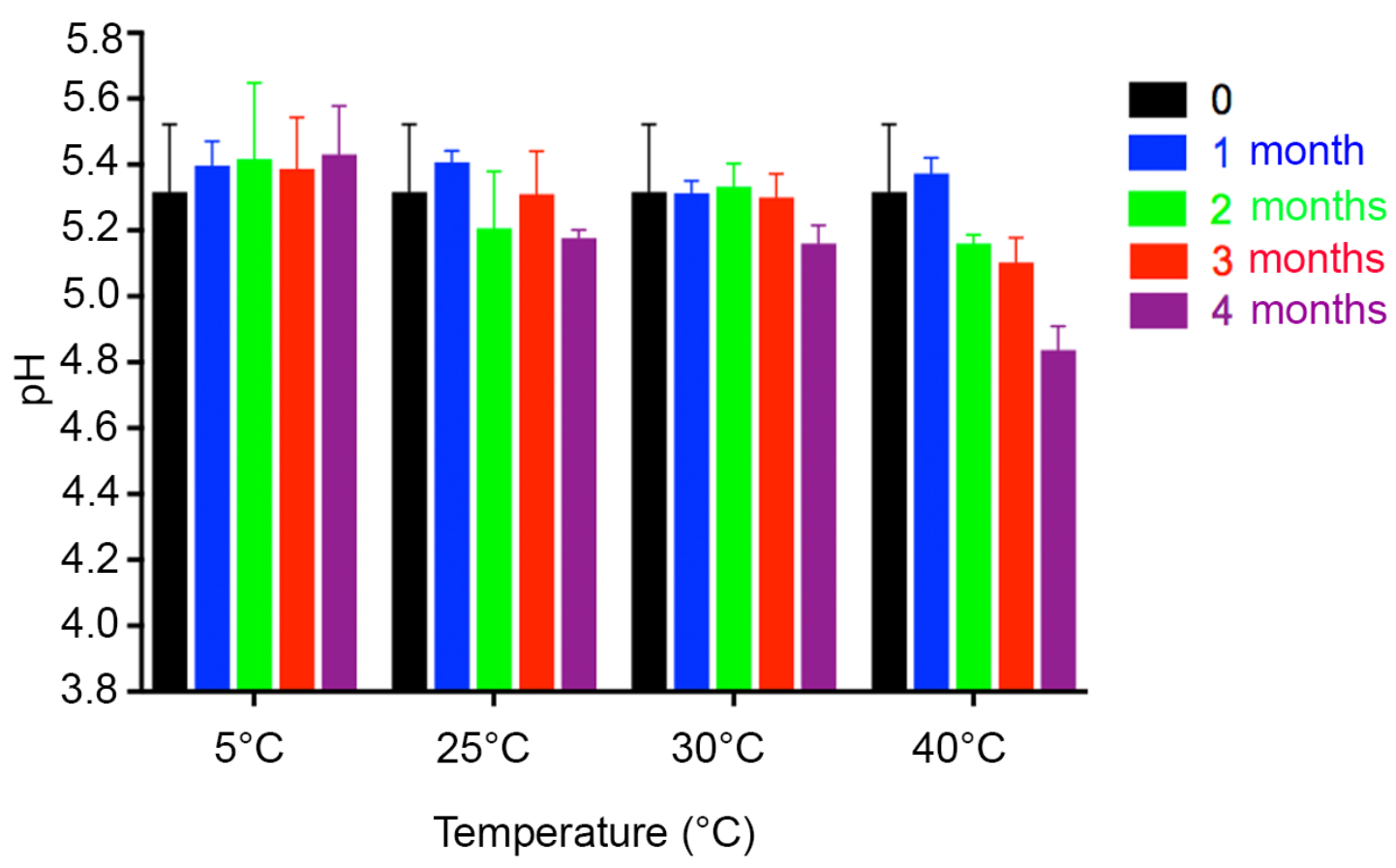

Figure 5-1. The pH of pregabalin-loaded Carbopol ME eye drops at different temperatures after four month of stability study

Data are expressed as mean $\pm \mathrm{SD} ; \mathrm{n}=3$.

There was no significant change $(\mathrm{p}>0.05)$ on the $\mathrm{pH}$ values during the different test periods except at $40^{\circ} \mathrm{C}$ after only four months of stability study $(\mathrm{p}<0.01)$. 
Table 5-1. The pH values of our developed pregabalin-loaded ME eye drops at different temperatures after four month of stability study

\begin{tabular}{lcccc}
\hline & \multicolumn{4}{c}{$\mathbf{p H}$} \\
\cline { 2 - 5 } Time (month) & $\mathbf{5}^{\circ} \mathbf{C}$ & $\mathbf{2 5}^{\circ} \mathbf{C}$ & $\mathbf{3 0}^{\circ} \mathbf{C}$ & $\mathbf{4 0}^{\circ} \mathbf{C}$ \\
\hline 0 & $5.32 \pm 0.21$ & $5.32 \pm 0.21$ & $5.32 \pm 0.21$ & $5.32 \pm 0.21$ \\
1 & $5.40 \pm 0.07$ & $5.41 \pm 0.04$ & $5.31 \pm 0.04$ & $5.37 \pm 0.05$ \\
2 & $5.42 \pm 0.23$ & $5.21 \pm 0.17$ & $5.33 \pm 0.10$ & $5.16 \pm 0.03$ \\
3 & $5.39 \pm 0.16$ & $5.31 \pm 0.13$ & $5.30 \pm 0.07$ & $5.10 \pm 0.08$ \\
4 & $5.43 \pm 0.15$ & $5.18 \pm 0.03$ & $5.16 \pm 0.06$ & $4.84 \pm 0.07$ \\
\hline
\end{tabular}

Table 5-2. Statistical comparisons among pH values of pregabalin-loaded Carbopol ME eye drops stored at four different temperatures and the initial pH value of the same formulation

\begin{tabular}{lcccc}
\hline & \multicolumn{4}{c}{ P-value } \\
\cline { 2 - 5 } Temperature & 1 month & 2 month & 3 month & 4 month \\
\hline $5^{\circ} \mathrm{C}$ & 0.7465 & 0.8608 & 0.9211 & 0.6242 \\
$25^{\circ} \mathrm{C}$ & 0.6709 & 0.8199 & 0.9999 & 0.4597 \\
$30^{\circ} \mathrm{C}$ & 0.9999 & 0.9998 & 0.9997 & 0.3701 \\
$40^{\circ} \mathrm{C}$ & 0.8990 & 0.6002 & 0.2380 & 0.0022 \\
\hline
\end{tabular}

P-value represents the outcome of Dunnett's multiple comparisons tests where we compare the $\mathrm{pH}$ values at each temperature with the initial $\mathrm{pH}$ values after the one-way ANOVA analysis.

The yellow color indicates there is a significant difference 


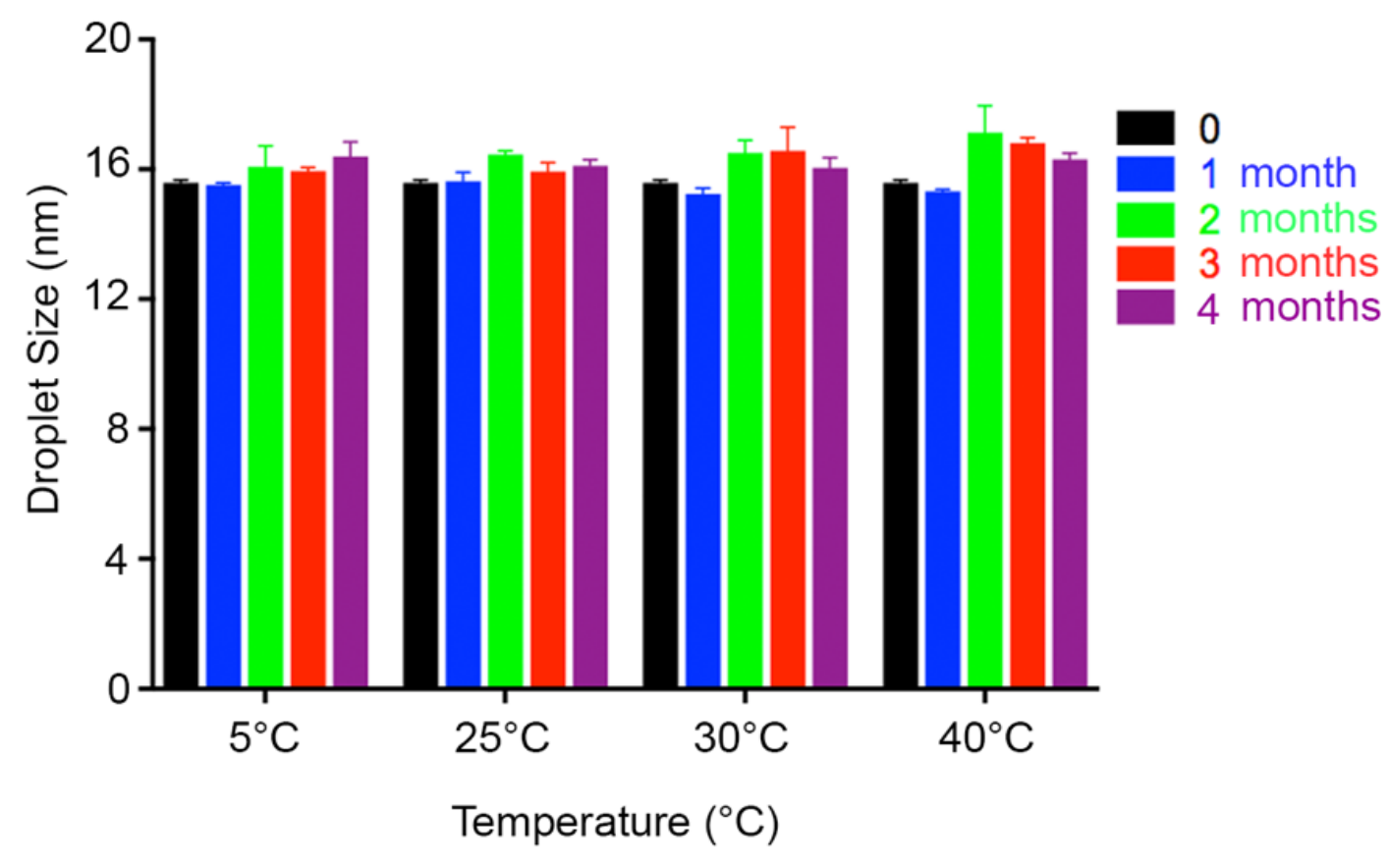

Figure 5-2. The mean droplet size of pregabalin-loaded ME eye drops at different temperatures after four month of stability study

Data are expressed as mean $\pm \mathrm{SD} ; \mathrm{n}=3$.

The initial average droplet size of our ME eye drops was $15.58 \pm 0.09$ while the highest measured value of the droplet size after four months of stability study was $17.14 \pm 0.82$. Hence our ME eye drops has a high stability because it maintained its small droplet size after four months of stability study at all the different studied temperatures. 
Table 5-3. The average droplet size of our prepared pregabalin-loaded ME eye drops at different temperatures after four month of stability study

\begin{tabular}{lcccc}
\hline & \multicolumn{4}{c}{ Droplet size (nm) } \\
\cline { 2 - 5 } Time (month) & $\mathbf{5}^{\circ} \mathbf{C}$ & $\mathbf{2 5}{ }^{\circ} \mathbf{C}$ & $\mathbf{3 0}^{\circ} \mathbf{C}$ & $\mathbf{4 0}^{\circ} \mathbf{C}$ \\
\hline 0 & $15.58 \pm 0.09$ & $15.58 \pm 0.09$ & $15.58 \pm 0.09$ & $15.58 \pm 0.09$ \\
1 & $15.52 \pm 0.07$ & $15.64 \pm 0.27$ & $15.25 \pm 0.17$ & $15.31 \pm 0.07$ \\
2 & $16.08 \pm 0.64$ & $16.45 \pm 0.11$ & $16.51 \pm 0.39$ & $17.14 \pm 0.82$ \\
3 & $15.95 \pm 0.11$ & $15.94 \pm 0.27$ & $16.57 \pm 0.73$ & $16.81 \pm 0.16$ \\
4 & $16.39 \pm 0.45$ & $16.12 \pm 0.19$ & $16.05 \pm 0.31$ & $16.31 \pm 0.18$ \\
\hline
\end{tabular}

Table 5-4. Statistical comparisons among droplet size values of pregabalinloaded Carbopol ME eye drops stored at four different temperatures and the initial droplet size value of the same formulation

\begin{tabular}{lcccc}
\hline & \multicolumn{4}{c}{ P-value } \\
\cline { 2 - 5 } Temperature & 1 month & 2 months & 3 months & 4 months \\
\hline $5^{\circ} \mathrm{C}$ & 0.9622 & 0.5824 & 0.5681 & 0.0151 \\
$25^{\circ} \mathrm{C}$ & 0.9761 & 0.1644 & 0.5869 & 0.1188 \\
$30^{\circ} \mathrm{C}$ & 0.0703 & 0.1358 & 0.0234 & 0.1804 \\
$40^{\circ} \mathrm{C}$ & 0.1663 & 0.0111 & 0.0064 & 0.0270 \\
\hline
\end{tabular}

The yellow color indicates there is a significant difference. 


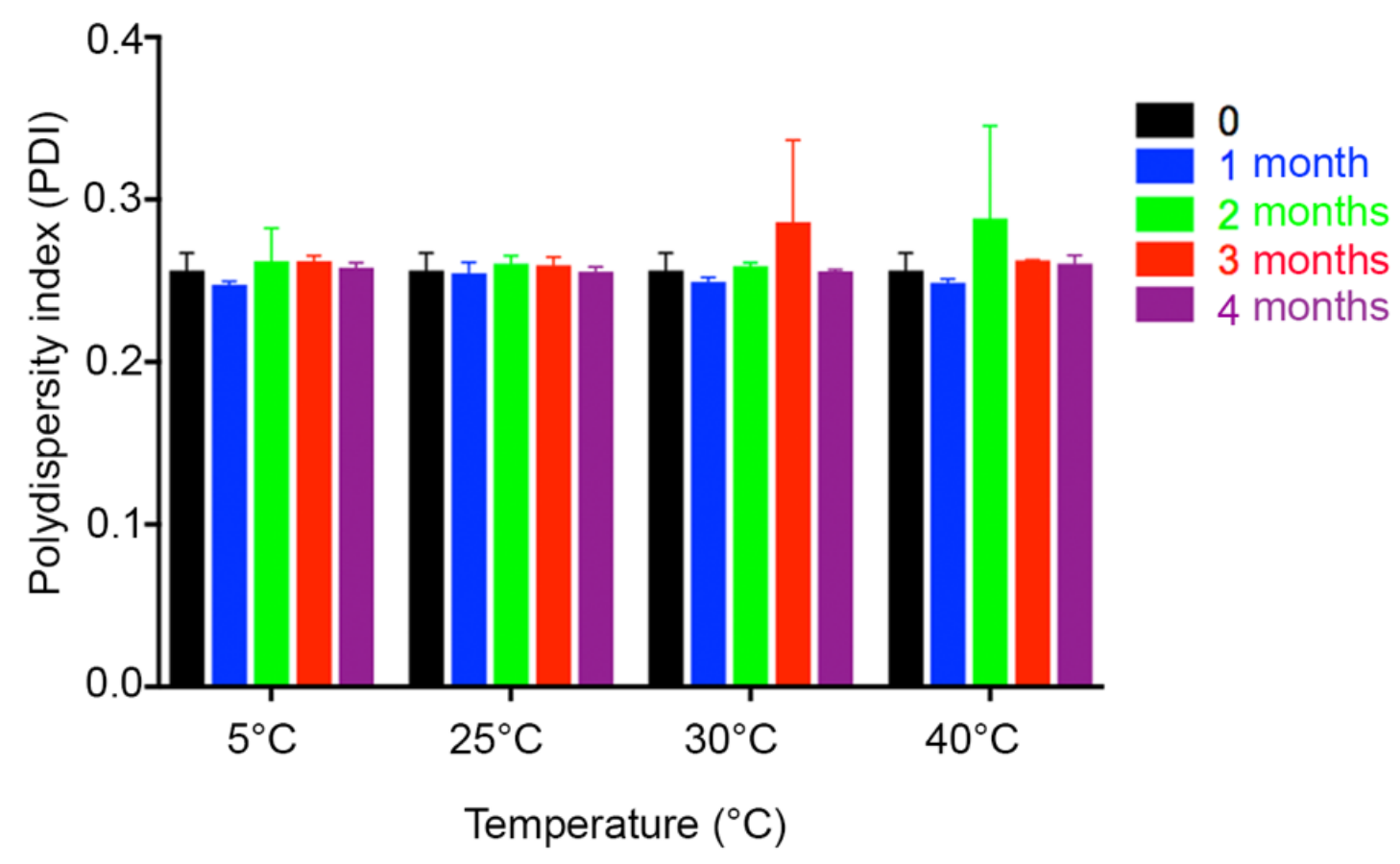

Figure 5-3. The PDI of pregabalin-loaded Carbopol ME eye drops at different temperatures after four month of stability study

Data are expressed as mean $\pm \mathrm{SD} ; \mathrm{n}=3$.

No significant difference ( $p>0.05$ ) was observed in the PDI at the four studied temperatures at all the time points. Such low PDI suggested that our ME formulation was uniform because a lower PDI value meant a higher homogeneous distribution and a highly stable ME.

Table 5-5. The average polydispersity index of our prepared pregabalin-loaded ME eye drops at different temperatures after four month of stability study

\begin{tabular}{lcccc}
\hline & \multicolumn{4}{c}{ PDI } \\
\cline { 2 - 5 } Time (month) & $\mathbf{5}^{\circ} \mathbf{C}$ & $\mathbf{2 5}^{\circ} \mathbf{C}$ & $\mathbf{3 0}^{\circ} \mathbf{C}$ & $\mathbf{4 0}^{\circ} \mathbf{C}$ \\
\hline 0 & $0.256 \pm 0.01$ & $0.256 \pm 0.01$ & $0.256 \pm 0.01$ & $0.256 \pm 0.01$ \\
1 & $0.248 \pm 0.00$ & $0.255 \pm 0.01$ & $0.249 \pm 0.00$ & $0.249 \pm 0.00$ \\
2 & $0.262 \pm 0.02$ & $0.261 \pm 0.01$ & $0.259 \pm 0.00$ & $0.288 \pm 0.06$ \\
3 & $0.262 \pm 0.00$ & $0.260 \pm 0.01$ & $0.286 \pm 0.05$ & $0.263 \pm 0.00$ \\
4 & $0.258 \pm 0.00$ & $0.256 \pm 0.00$ & $0.256 \pm 0.00$ & $0.261 \pm 0.01$ \\
\hline
\end{tabular}


was observed at $40^{\circ} \mathrm{C}(0.288 \pm 0.06)$ compared to the initial PDI value $(0.256 \pm 0.01)$. No significant difference ( $>0.05)$ (Table 5-6) was observed in the PDI at the four studied temperatures at all the time points. Such low PDI suggested that our ME formulation was uniform because a lower PDI value meant a higher homogeneous distribution and a highly stable ME (59).

Figure 5-4 and Table 5-7 illustrated the obtained results of the stability study zeta potential at different time points. After four months of stability study the highest measured negative zeta potential was observed at $40^{\circ} \mathrm{C}(48.36 \pm 0.40)$ compared to the initial zeta potential value (31.42 \pm 1.14$)$. After one month, there was no significant change in the measured zeta potential $(\mathrm{p}>0.05)$ (Table 5-8). However, there was a significant change after two months at both $30^{\circ} \mathrm{C}(\mathrm{p}<0.001)$ and $40^{\circ} \mathrm{C}(\mathrm{p}<0.001)$. There was no significant change after three months at $5^{\circ} \mathrm{C}(\mathrm{p}>0.05)$, while there was a significant alteration at the other three studied temperatures $(p<0.001)$. Furthermore, after four month of stability study there was no significant change in the zeta potential at $5^{\circ} \mathrm{C}$, $25^{\circ} \mathrm{C}$ and $30^{\circ} \mathrm{C}(\mathrm{p}>0.05)$, while there was a significant change at only $40^{\circ} \mathrm{C}(\mathrm{p}<0.05)$. In general, for any colloidal system the surface charge of very important factor to achieve a long-term stability as it resulted in repulsion between droplets and prevented their coalescence upon standing. Meanwhile the higher the surface charge the higher the system stability whatever the charge type either positive or negative.

\section{Drug content}

Table 5-9. presented the results of the drug content as pregabalin-percentage amount remained in the prepared ME eye drops after storage at different temperatures for four months. After one and two month of stability study, there was no significant change in the measured drug content ( $p>0.05$ ) (Table 5-10) at the four different studied temperatures. However, there was a significant change in the drug content after three months at only $40^{\circ} \mathrm{C}(\mathrm{p}<0.05)$. Also, after four months, there was a significant change at the four different studied temperatures $\left(p<0.01\right.$ at $5^{\circ} \mathrm{C}$ and $\mathrm{p}<0.001$ at $25^{\circ} \mathrm{C}, 30^{\circ} \mathrm{C}$ and $40^{\circ} \mathrm{C}$ ). From the obtained drug content results, it could therefore be suggested that our pregabalin-loaded ME eye drops should be stored at either $5^{\circ} \mathrm{C}$ or $25^{\circ} \mathrm{C}$ because the drug content didn't change significantly and more than $90 \%$ of the pregabalin concentration remained in the formulation. Similarly, Zhu et al., (122) reported that penciclovir-loaded ME was stable at $4^{\circ} \mathrm{C}$ for 3 months with no significant change in physicochemical properties. Furthermore, previous studies proved the stability of ME-based dexamethasone eye drops for 3 months at ${ }^{\circ} \mathrm{C}(74)$.

It has been reported that at least four half-lives are required to calculate the shelf life and the expiration date as well as the mechanism of drug degradation (123) so we were unable to determine these parameters yet as we did the stability study for four months where the formulation showed a good stability. The stability investigation of the formulation is still ongoing. 
Table 5-6. Statistical comparisons among PDI values of pregabalin-loaded Carbopol ME eye drops stored at four different temperatures and the initial droplet size value of the same formulation

\begin{tabular}{lcccc}
\hline & \multicolumn{4}{c}{ P-value } \\
\cline { 2 - 5 } Temperature & 1 month & 2 months & 3 months & 4 months \\
\hline $5^{\circ} \mathrm{C}$ & 0.2741 & 0.9965 & 0.9940 & 0.9877 \\
$25^{\circ} \mathrm{C}$ & 0.9841 & 0.9990 & 0.9992 & 0.9988 \\
$30^{\circ} \mathrm{C}$ & 0.4356 & 0.9998 & 0.3777 & 0.9998 \\
$40^{\circ} \mathrm{C}$ & 0.3872 & 0.4550 & 0.9916 & 0.7862 \\
\hline
\end{tabular}

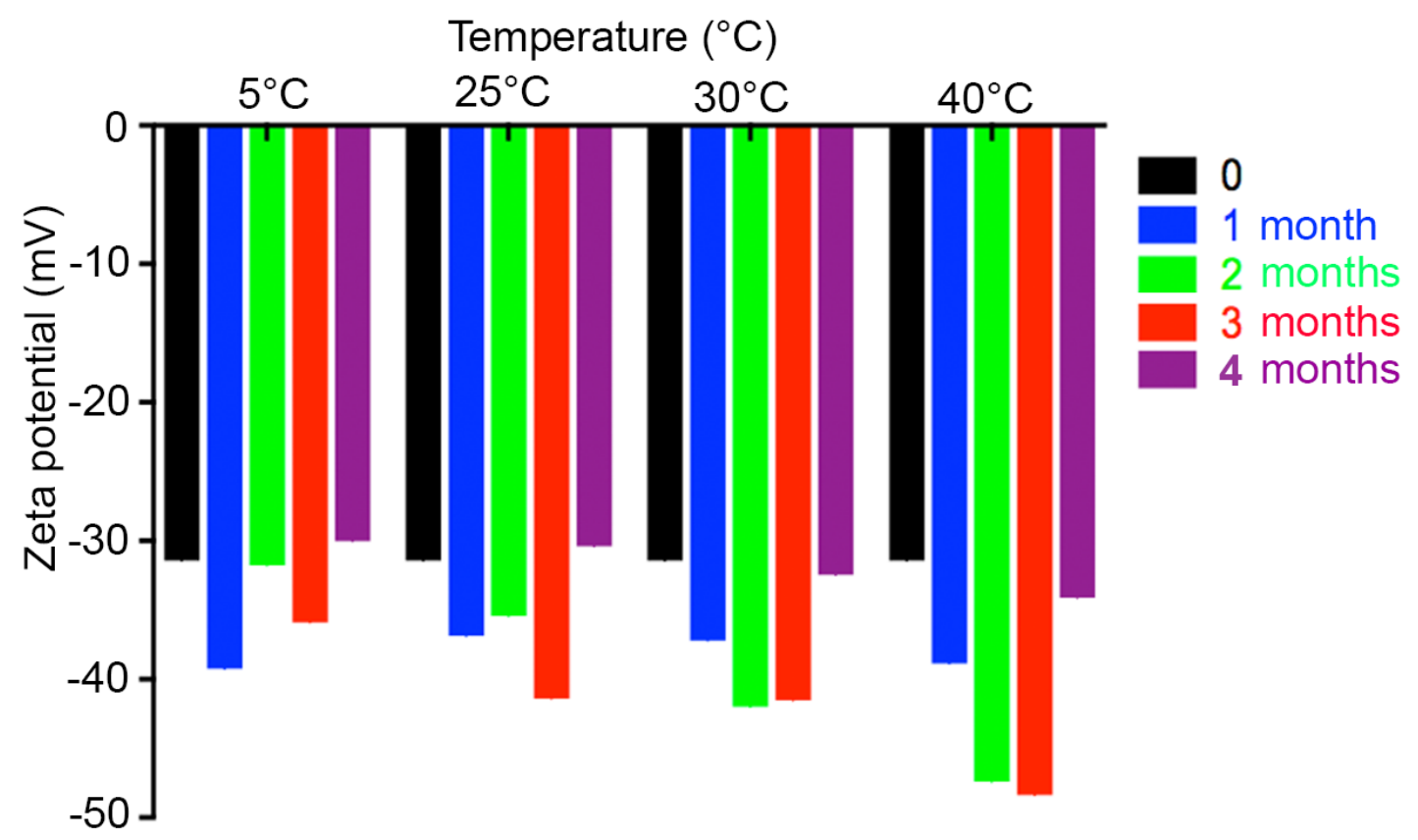

Figure 5-4. The zeta potential of pregabalin-loaded Carbopol ME eye drops at different temperatures after four months of stability study

Data are expressed as mean $\pm \mathrm{SD} ; \mathrm{n}=3$.

After four months of stability study, there was no significant change in the zeta potential at $5^{\circ} \mathrm{C}, 25^{\circ} \mathrm{C}$ and $30^{\circ} \mathrm{C}(\mathrm{p}>0.05)$ while there was a significant change at only $40^{\circ} \mathrm{C}$ $(\mathrm{p}<0.05)$. 
Table 5-7. The average zeta potential of our prepared pregabalin-loaded ME eye drops at different temperatures after four month of stability study

\begin{tabular}{lcccc}
\hline & \multicolumn{4}{c}{ Zeta potential (mV) } \\
\cline { 2 - 5 } Time (month) & $\mathbf{5}^{\circ} \mathbf{C}$ & $\mathbf{2 5}^{\circ} \mathbf{C}$ & $\mathbf{3 0}^{\circ} \mathbf{C}$ & $\mathbf{4 0}^{\circ} \mathbf{C}$ \\
\hline 0 & $-31.42 \pm 1.14$ & $-31.42 \pm 1.14$ & $-31.42 \pm 1.14$ & $-31.42 \pm 1.14$ \\
1 & $-39.27 \pm 7.90$ & $-36.89 \pm 1.43$ & $-37.20 \pm 3.46$ & $-38.90 \pm 1.31$ \\
2 & $-31.77 \pm 2.49$ & $-35.41 \pm 2.44$ & $-41.99 \pm 0.90$ & $-47.38 \pm 1.74$ \\
3 & $-35.88 \pm 2.09$ & $-41.39 \pm 1.35$ & $-41.52 \pm 3.78$ & $-48.36 \pm 0.40$ \\
4 & $-30.02 \pm 1.83$ & $-30.40 \pm 0.32$ & $-32.46 \pm 0.84$ & $-34.12 \pm 0.90$ \\
\hline
\end{tabular}

Table 5-8. Statistical comparisons among the zeta potential values of pregabalinloaded Carbopol ME eye drops stored at four different temperatures and the initial zeta potential value of the same formulation

\begin{tabular}{lllll}
\hline & \multicolumn{4}{c}{ P-value } \\
\cline { 2 - 5 } Temperature & 1 month & 2 months & 3 months & 4 months \\
\hline $5^{\circ} \mathrm{C}$ & 0.1069 & 0.9977 & 0.0798 & 0.3977 \\
$25^{\circ} \mathrm{C}$ & 0.3232 & 0.0764 & 0.0006 & 0.6428 \\
$30^{\circ} \mathrm{C}$ & 0.2796 & 0.0002 & 0.0005 & 0.6350 \\
$40^{\circ} \mathrm{C}$ & 0.1279 & 0.0001 & 0.0001 & 0.0450 \\
\hline
\end{tabular}

The yellow color indicates there is a significant difference. 
Table 5-9. The percentage amount remaining of pregabalin in our prepared pregabalin-loaded ME eye drops at different temperatures after four month of stability study

\begin{tabular}{lcccc}
\hline & \multicolumn{4}{c}{ The percentage amount remaining of pregabalin } \\
\cline { 2 - 5 } Time (month) & $\mathbf{5}^{\circ} \mathbf{C}$ & $\mathbf{2 5} \mathbf{C}$ & $\mathbf{3 0}^{\circ} \mathbf{C}$ & $\mathbf{4 0}^{\circ} \mathbf{C}$ \\
\hline $\mathbf{0}$ & $100 \pm 00$ & $100 \pm 00$ & $100 \pm 00$ & $100 \pm 00$ \\
$\mathbf{1}$ & $100.00 \pm 1.23$ & $100.00 \pm 0.88$ & $100.00 \pm 1.98$ & $100.00 \pm 4.17$ \\
$\mathbf{2}$ & $104.09 \pm 1.3$ & $103.48 \pm 1.9$ & $100.55 \pm 1.88$ & $100.31 \pm 2.9$ \\
$\mathbf{3}$ & $96.76 \pm 4.9$ & $92.21 \pm 2.1$ & $86.25 \pm 1.2$ & $82.54 \pm 12.6$ \\
$\mathbf{4}$ & $87.19 \pm 2.51$ & $81.60 \pm 5.94$ & $78.21 \pm 3.87$ & $48.83 \pm 1.97$ \\
\hline
\end{tabular}

Table 5-10. Statistical comparisons among the percentage amount remaining of pregabalin values of pregabalin-loaded Carbopol ME eye drops stored at four different temperatures and the initial value of the same formulation

\begin{tabular}{lcccc}
\hline & \multicolumn{4}{c}{ P-value } \\
\cline { 2 - 5 } Temperature & 1 month & 2 months & 3 months & 4 months \\
\hline $\mathbf{5}^{\circ} \mathbf{C}$ & 0.9999 & 0.0702 & 0.9123 & 0.0013 \\
$\mathbf{2 5}^{\circ} \mathbf{C}$ & 0.9999 & 0.1328 & 0.3877 & 0.0001 \\
$\mathbf{3 0}^{\circ} \mathbf{C}$ & 0.9999 & 0.9878 & 0.0642 & 0.0001 \\
$\mathbf{4 0}^{\circ} \mathbf{C}$ & 0.9999 & 0.9986 & 0.0192 & 0.0001 \\
\hline
\end{tabular}

The yellow color indicates there is a significant difference. 


\section{In vitro release}

During the stability study of our pregabalin-loaded ME eye drops we thought to determine the ability of our multiple W/O/W ME system to maintain the sustained release pattern of pregabalin over the different studied periods at different temperatures. Figure 5-5 showed the initial cumulative amount released of pregabalin up to $24 \mathrm{~h}$ (Figure 5-5

A) and that after 1, 2, 3 and 4 months of stability study (Figure 5-5 B, C, D and E).

Table 5-11 presented the statistical analysis of data at three time points during the in vitro release- $2 \mathrm{~h}, 8 \mathrm{~h}$ and $24 \mathrm{~h}$. After four months, there was no significant difference $(p>0.05)$ in the cumulative amount released of pregabalin from our ME eye drops stored at the four different temperatures compared to the initial data. However, after one, two and three months there was only one or two points that showed significant change (Table 5-11). 


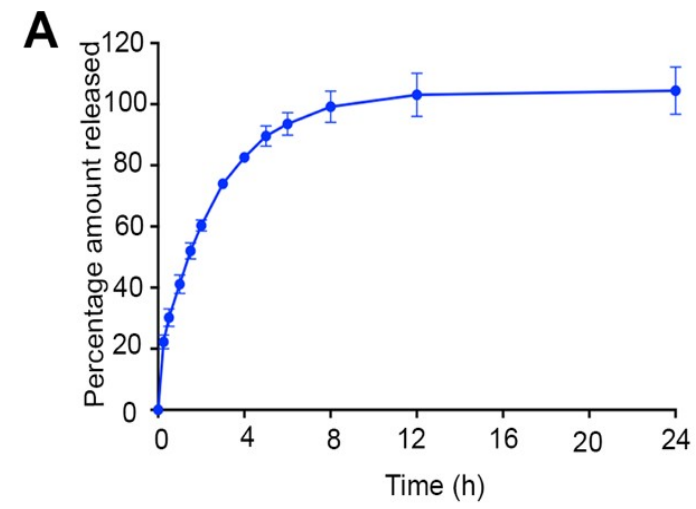

B

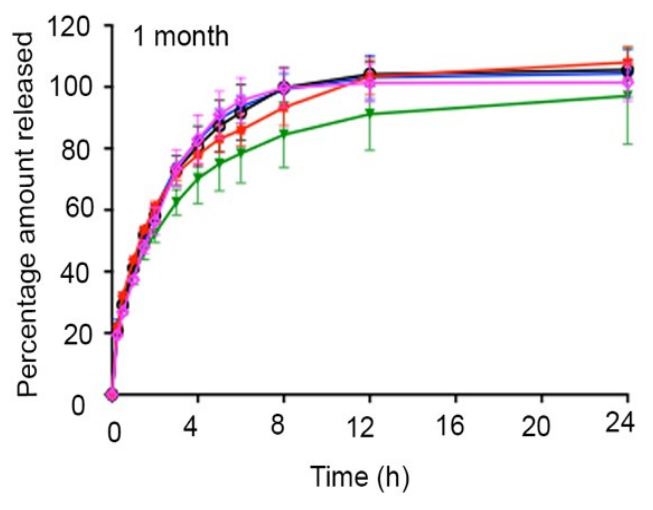

D

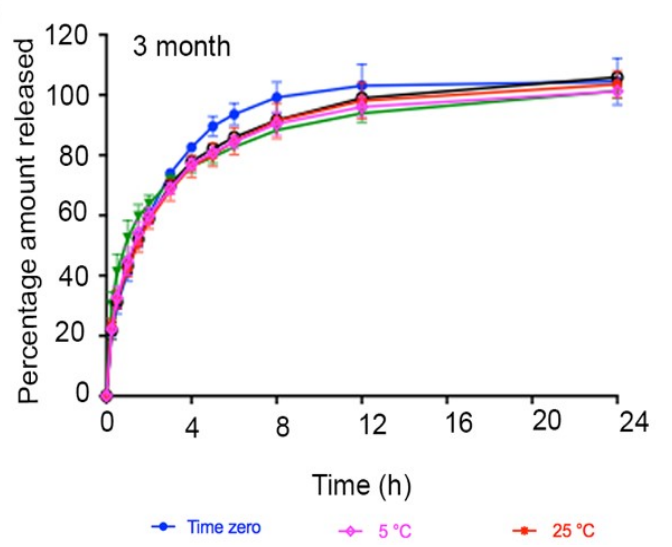

C

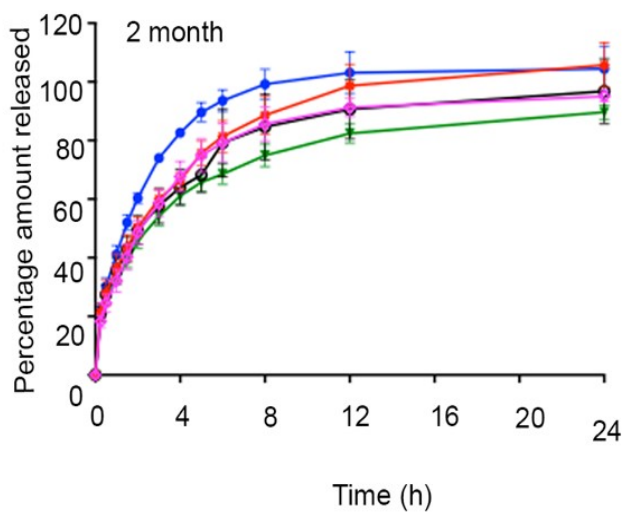

E

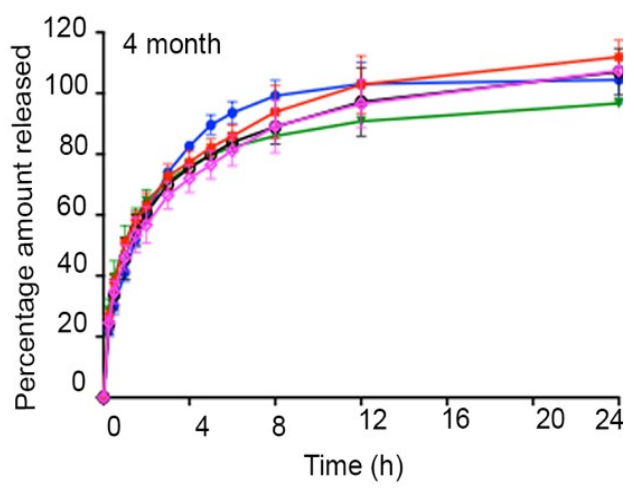

Figure 5-5. Cumulative amount released (\%) of pregabalin from Carbopol ME eye drops $(0.6 \%)$ at different temperatures after four month of stability study

Data are expressed as mean $\pm \mathrm{SD} ; \mathrm{n}=3$

After four months, there was no significant difference $(\mathrm{p}>0.05)$ in the cumulative amount released of pregabalin from our ME eye drops stored at the four different temperatures compared to the initial data. However, after one, two and three months there was only one or two points that showed significant change. 
Table 5-11. Statistical comparisons among the cumulative amounts released of pregabalin after four months of stability and the initial values of the same formulation

\begin{tabular}{|c|c|c|c|c|c|c|c|c|c|c|c|c|}
\hline \multirow[b]{3}{*}{ Temperature } & \multicolumn{12}{|c|}{ P-value } \\
\hline & \multicolumn{3}{|c|}{1 month } & \multicolumn{3}{|c|}{2 months } & \multicolumn{3}{|c|}{3 months } & \multicolumn{3}{|c|}{4 months } \\
\hline & $2 \mathrm{~h}$ & $8 h$ & $24 h$ & 2h & $8 h$ & $24 h$ & $2 \mathrm{~h}$ & $8 \mathrm{~h}$ & $24 h$ & $2 \mathrm{~h}$ & $8 h$ & $24 h$ \\
\hline $5^{\circ}$ & 0.3042 & 0.9999 & 0.9790 & 0.0071 & 0.1223 & 0.3494 & 0.9945 & 0.0634 & 0.7507 & 0.6733 & 0.2393 & 0.9105 \\
\hline $25^{\circ} \mathrm{C}$ & 0.9961 & 0.7161 & 0.9627 & 0.0195 & 0.3445 & 0.9987 & 0.7015 & 0.1055 & 0.9965 & 0.8675 & 0.7128 & 0.3377 \\
\hline $30^{\circ} \mathrm{C}$ & 0.7722 & 0.9999 & 0.9994 & 0.0109 & 0.0951 & 0.5088 & 0.9028 & 0.1158 & 0.9806 & 0.9998 & 0.2317 & 0.9364 \\
\hline $40^{\circ} \mathrm{C}$ & $0.0192 *$ & 0.0933 & 0.6987 & 0.0015 & 0.0072 & 0.0876 & 0.3149 & 0.0223 & 0.7784 & 0.5769 & 0.0975 & 0.3103 \\
\hline
\end{tabular}

The yellow color indicates there is a significant difference. 


\section{CHAPTER 6. DISCUSSION AND CONCLUSION}

\section{Future Directions}

Although topical application of eye drops is an ideal approach to treat various eye diseases affecting the anterior segment of the eye, several drawbacks of these conventional ophthalmic formulations arise because of extensive precorneal loss that will end with low bioavailability. Therefore, several strategies have been designed to address these drawbacks as an attempt to improve bioavailability of topically applied drugs. In the pharmaceutical field, microemulsion have been widely used to enhance corneal drug penetration, increase drug loading, improve drug stability and bioavailability. Glaucoma as a progressive optic neuropathy with an elevated intraocular pressure as a major risk factor is the second cause of blindness in the world. Hence, lowering the IOP is the only target of the currently available glaucoma drugs. In our recently published work, we identified a new candidate gene, Cacna2dl, that modulates IOP and a promising therapeutic, pregabalin, which binds to CACNA2D1 protein and lowers IOP significantly in both mice and rabbits. Although in our study we proved the IOP-lowering efficiency of pregabalin in a dose dependent manner, several dosings of simple aqueous pregabalin eye drops is required because of short duration of action. Unfortunately, the hydrophilicity of the drug might be the main reason for such a short duration of action. We hypothesized that a new topical microemulsion bioadhesive ophthalmic formulation can sustain the release of pregabalin as a novel glaucoma therapy. Thus, in this study we wanted to develop an optimized ME-based ocular preparation of pregabalin for glaucoma and test its ability to sustain the drug release as a once-daily eye drop.

\section{Aim 1: To optimize and develop a multiple $\mathrm{W} / \mathrm{O} / \mathrm{W}$ microemulsion eye drops}

Using aqueous titration method and ternary\& pseudo-ternary phase diagram construction, we were able to determine the proper weight ratios of the oil phase, aqueous phase and surfactant $\&$ co-surfactant to prepare the primary $\mathrm{W} / \mathrm{O}$ microemulsion. By selecting surfactant $\&$ co-surfactant with a hydrophilic-lipophilic balance values of $\leq 5$ we were able to decrease the number of screened surfactants \& co-surfactants to save our time efficiently. Hence, after a number of systems were tested a system including $30 \%$ $\mathrm{w} / \mathrm{w}$ of Labrafac lipophile WL1349 as the oil phase, MilliQ water $(20 \% \mathrm{w} / \mathrm{w})$ as the aqueous phase and 50\% w/w of Capryol $90 \&$ soybean lecithin, 1:1 mixture as the nonionic surfactant/cosurfactant mixture, was prepared. To select the proper hydrophilic surfactant \& co-surfactant to prepare the external aqueous phase, different combination systems with high HLB values $(\geq 12)$ were tested to prepare the multiple $\mathrm{W} / \mathrm{O} / \mathrm{W} \mathrm{ME}$ disperse system. Also, one of the strategies that we used for these studies was the incorporation of a bioadhesive polymer in the external aqueous phase of our multiple $\mathrm{ME}$ to increase the viscosity and the precorneal contact time of our ocular ME eye drops.

In this work, we aimed to slow the release of pregabalin and hence increase its duration of action as an effective glaucoma therapy. Hence, we have incorporated $40 \%$ of 
the predetermined pregabalin dose in the innermost aqueous phase of our developed multiple $\mathrm{W} / \mathrm{O} / \mathrm{W}$ ME system while incorporating the remaining dose (60\%) in the external polymeric aqueous phase. By such a strategy, a slow pregabalin release with a prolonged contact time will achieve a long duration of action after a single dose application.

Our Aim 1 studies were innovative for at least the following five reasons:

1. We have used Labrafac lipophile WL 1349, a medium chain triglyceride ester as the oil phase during the development of our ME system because of having a high shelf stability and less susceptibility to oxidation compared to long chain triglycerides.

2. We have utilized a non-ionic surfactant/cosurfactant mixture-Capryol $90 \&$ soybean lecithin, 1:1 mixture — which has a high compatibility with the oil phase.

3. We have included a bioadhesive polymer - chitosan, sodium alginate or Carbopol 981 - in the external aqueous phase to insure a prolonged contact time after a single dose application.

4. We have designed the incorporation of pregabalin dose by such away that we can induce a short onset with a longer duration of action as we incorporated only $40 \%$ of the dose in the innermost aqueous phase while incorporating the other $60 \%$ of the dose in the external aqueous phase.

5. We have successfully developed a multiple W/O/W ME bioadhesive eye drops using few synthesis steps with an ease preparation and scaling up with no need for a specific type of equipment.

\section{Aim 2: To characterize the prepared multiple $\mathrm{W} / \mathrm{O} / \mathrm{W}$ microemulsion eye drops}

Due to the several drawbacks of eye drops which are the most currently available ocular drug delivery system, we aimed to overcome these limitations by addressing two important criteria. The first was to sustain the drug release; and the second was to improve the formulation bioadhesiveness to prolong its corneal contact time. Here, in our research, we strived to achieve these two targets by incorporating pregabalin in a multiple $\mathrm{W} / \mathrm{O} / \mathrm{W} \mathrm{ME}$ that included a bioadhesive polymer.

Most ME published studies showed how ME had been successfully used to improve the bioavailability of poorly water-soluble drugs by increasing their solubility and hastening their release and absorption $(70,124,125)$. On the contrary, in our project we succeeded to use ME to slow the drug release and delay its corneal transport. Because our drug is water soluble, we modified the ME composition to take the advantages of both ME types; the slow release behavior of the W/O type and the aqueous sensation of the $\mathrm{O} / \mathrm{W}$ type. Lastly, using our multiple $\mathrm{ME}$ we will be able to develop a promising 
novel extended release formulation to deliver pregabalin (a new glaucoma medication) (24) with an expected excellent patient satisfaction.

During the preparation of our multiple ME, pregabalin ( $40 \%$ of the dose) was dissolved in the internal aqueous phase and then emulsified in the oil phase to produce the primary $\mathrm{W} / \mathrm{O} \mathrm{ME}$. The produced $\mathrm{W} / \mathrm{O} \mathrm{ME}$ was further emulsified in an aqueous viscous polymeric solution to produce the final multiple $\mathrm{W} / \mathrm{O} / \mathrm{W}$ bioadhesive $\mathrm{ME}$. In this study, we hypothesized that the addition of specifically selected polymers (126) that have excellent bioadhesive properties - chitosan, sodium alginate and Carbopol—will increase the precorneal contact time of our ME eye drops and therefore can increase the pregabalin duration of action. Using this specific design, the drug was located in two different phases within the multiple ME; the innermost aqueous layer and the external polymeric aqueous phase. So, to be released, the drug from the internal aqueous phase must pass through two interfaces the inner $\mathrm{W} / \mathrm{O}$ interface and the outer $\mathrm{O} / \mathrm{W}$ interface after which it has to diffuse through the viscous polymeric matrix to be ready for transport through the corneal epithelium layer. Moreover, an initial fast release might be an outcome of the presence of this $60 \%$ of the drug dose in the external aqueous phase, which was ready to be released after diffusion within the polymeric network. Using such a strategy, we successfully delayed and sustained the pregabalin release from our optimized ME-based formulation while maintaining the advantages of simple easy application into the eye. This project represents a novel application of ME in ocular drug delivery for water-soluble drugs.

Although the pregabalin corneal permeability has not been studied yet, being a class I drug according to the BCS provided us with an expectation of a relatively high corneal permeability as well. Here, we used fresh isolated New Zealand white rabbit corneas to measure pregabalin corneal permeability from our developed ME eye drops and pregabalin aqueous solution. By this way we can investigate if our ME system affect the drug corneal permeability or not. We used BSS-PLUS ${ }^{\circledR}$ irrigating solution as a receptor medium during the study. Such medium would be ideal to keep the cornea alive during the whole experiment (6h), since this medium has a similar composition to aqueous humor and was commonly used as a sterile intraocular irrigating solution due to its ability to maintain the anatomic and physiologic integrity of intraocular tissues. In our study as we expected pregabalin proved to have a relatively high corneal permeability that will result in a high ocular bioavailability.

Since ME as a colloidal drug carrier was generally used to enhance drug corneal penetration for drugs that have low corneal permeability, in our study our ME formulation could maintain the high permeability associated with pregabalin with no significant difference.

Because we want to investigate the IOP-lowering effect of our new developed ME eye drops in Dutch-belted rabbits, we wanted to test first if CACNA2D1 - the pregabalin target protein-is expressed in these animals eye tissues and if yes which tissues of the eye. Similarly, the IHC results in Dutch belted rabbit eyes were in agreement with what we obtained before using both mouse and human donor eyes (24). Since CACNA2D1 
was highly expressed in both the ciliary body (non-pigmented epithelium) and the trabecular meshwork so the mechanism through which pregabalin decreased the IOP might be through decreasing the aqueous humor production by the ciliary body or increasing its drainge through the trabecular meshwork or by both mechanisms.

Our Aim 2 studies were innovative for the following reasons:

1. We have developed a multiple ME eye drops as a promising carrier that succeeded to sustain the release of pregabalin up to $24 \mathrm{~h}$.

2. By using isolated New Zealand white rabbit corneas, we were able to measure for the first time pregabalin high corneal permeability that was successfully maintained by our ME new formulations.

3. We have proved the localization of pregabalin target protein in areas associated with IOP modulation - the non-pigmented epithelium of ciliary body and the trabecular meshwork.

4. We have utilized the IHC results to suggest the mechanism through which pregabalin successfully decreased the IOP as a promising glaucoma therapy.

5. We developed a novel and fully validated ME-based pregabalin eye drops that can be used as a promising carrier for other water-soluble drugs.

\section{Aim 3: To determine the safety, in vivo efficacy and cell toxicity of our prepared multiple $\mathrm{W} / \mathrm{O} / \mathrm{W}$ microemulsion eye drops in Dutch belted rabbits and a human corneal limbal epithelial cell line}

Due to the slow progressive loss of vision with no obvious symptoms in most cases of glaucoma, a main focus of interest should be to develop a once-daily glaucoma eye drops to help to control the elevated IOP with a high patient convenience. Since our IHC results illustrated the localization of pregabalin target protein in Dutch belted eye tissues that modulate IOP, we used these animals to determine if our sustained release ME eye drops could maintain the IOP at a lower level for longer period of time after a single dose application. In addition, Dutch belted rabbits have a high IOP baseline before the application of our eye drops which can mimic the situation in case of glaucoma patients. In this study, we wanted to test if there was a significant difference between our multiple ME eye drops and the pregabalin aqueous eye drops in our previous studies by calculating the different pharmacodynamic parameters - $\mathrm{T}_{\max }, \% \mathrm{I}_{\max }, \mathrm{T}_{\text {end }}$ and $\mathrm{AUC}$-at the end of the experiment.

Also, to test the safety of our ME eye drops, we examined rabbits eyes every hour for any sign of irritation such as redness, tearing, conjunctival swelling or corneal swelling as well as slit-lamp examination at the end of the study. In this study, a single drop of our developed pregabalin-loaded Carbopol ME eye drops induced $42.3 \pm 2.6 \%$ 
IOP reduction that returned to baseline at $32.7 \pm 1.3 \mathrm{~h}$ from application $(\mathrm{AUC}=169.9 \pm 13.4$ mmHg.h). However, in the absence of the ME system, the same drug produced only $29.4 \pm 1.4 \%$ IOP reduction that returned to baseline at $9.3 \pm 0.7 \mathrm{~h}$ (AUC $=39 \pm 4 \mathrm{mmHg} . \mathrm{h}$ ). Our results are expected to fundamentally advance the field of glaucoma ocular therapy.

Our Aim 3 studies were innovative for the following reasons:

1. We have successfully developed a once-daily ME eye drops as a new glaucoma therapy.

2. Our developed ME eye drops have a good safety in both the intact animalsDutch belted rabbits - and the HCLE cell line (in vitro cytotoxicity).

\section{Aim 4: To determine the physical and chemical stability of our ME eye drops}

Because our developed pregabalin-loaded Carbopol 981 ME eye drops is a new drug product so to establish the different storage conditions we wanted to test its stability under different environmental conditions. So, we used the centrifugation tests as well as the freeze-thaw cycles test to evaluate the physical stability of our new pharmaceutical product. Also, we can easily study the chemical stability of our formulation by accelerated, intermediate and long-term stability tests at $40^{\circ} \mathrm{C}, 30^{\circ} \mathrm{C}, 25^{\circ} \mathrm{C}$ and $5^{\circ} \mathrm{C}$, respectively. In this study the measurement of droplet size, PDI and zeta potential was a good tool to assess the ME system stability under each specific storage condition. In addition, the drug content was an important parameter to test the stability of the drug substance and determine any degree of degradation.

Our Aim 4 studies were innovative for the following reasons:

1. Our pregabalin-loaded Carbopol ME eye drops has an excellent physical stability under intermediate and long-term condition-at $5^{\circ} \mathrm{C}, 25^{\circ} \mathrm{C}, 30^{\circ} \mathrm{C}$ - for the whole period of stability study (4 months).

2. Our new pharmaceutical ME eye drops was able to maintain its small droplet size and low PDI during the whole stability study (4 months) which confirmed its high stability.

3. We provide the pharmaceutical industry with a novel once-daily glaucoma ME eye drops with a good chemical stability for 3 months at both $5^{\circ} \mathrm{C}$ and $25^{\circ} \mathrm{C}$.

\section{Conclusion}

Glaucoma is the leading cause of irreversible blindness worldwide. Elevated IOP is one of the most significant risk factors contributing to visual field loss in POAG, the most common form of glaucoma. Maintaining the IOP at normal level is very important 
during the management of the disease to suppress or slow down the disease progression. Because of the importance of a tightly maintained IOP, coupled with the fact that IOP can be medically controlled, IOP reduction is the first-line therapeutic option in glaucoma. Unfortunately, current medications suffer from poor patient compliance due to their short half-life and low residence time on the cornea, which require topical application multiple times per day. Also they are associated with reduced efficacy and systemic side effects. All these gaps represent enormous unmet needs. In this study we succeeded to address most of these limitations associated with current drug therapies by developing our IOP-lowering sustained release bioadhesive topical ME eye drops. Characterization results proved that our new formulations were suitable for topical ophthalmic use.

Despite being water-soluble, our ME formulations could enable pregabalin to be administered as a once-daily eye drops. In vitro release study showed that our ME sustained the drug release up to $24 \mathrm{~h}$. Moreover, cytotoxicity study using MTT assay proved the non-cytotoxicity of our ophthalmic formulations to the human corneal limbal epithelial cell line. Also, our formulations had a reasonable viscosity, which was very important to prevent their precorneal loss from the eye surface for a while in order to give the chance for the bioadhesive interaction to occur to help the formulations to stick to the eye surface for more than one day. Furthermore, the bioadhesive study proved that the ME possessed high bioadhesive force than the controls. In addition, the corneal permeability experiment showed that our ME eye drops could maintain the high pregabalin corneal permeability (BCS class I drug) while controlling the drug release. Also, immunohistochemistry results demonstrated that CACNA2D1 was prominently localized in areas associated with aqueous humor modulation; both the CB and TM tissues of Dutch belted rabbits eyes. Moreover, the in vivo study of pregabalin ME eye drops on Dutch belted rabbits proved that only one drop of the ME could keep the IOP at lower level for up to $32.7 \pm 1.3 \mathrm{~h}$ with $42.3 \pm 2.6 \%$ IOP reduction, which suggested its oncedaily application as a new glaucoma therapy. Finally, we can conclude that our ME eye drops could serve as a novel promising ocular drug delivery system that could sustain the drug release and prolong the duration of action not only for pregabalin but also for any other water-soluble drugs. 


\section{LIST OF REFERENCES}

1. Bourlais CL, Acar L, Zia H, Sado PA, Needham T, Leverge R. Ophthalmic drug delivery systems--recent advances. Prog Retin Eye Res. 1998;17(1):33-58.

2. Lin HR, Sung KC, Vong WJ. In situ gelling of alginate/pluronic solutions for ophthalmic delivery of pilocarpine. Biomacromolecules. 2004;5(6):2358-65. doi: $10.1021 / \mathrm{bm} 0496965$.

3. Urtti A. Challenges and obstacles of ocular pharmacokinetics and drug delivery. Adv Drug Deliv Rev. 2006;58(11):1131-5. doi: 10.1016/j.addr.2006.07.027.

4. Gratieri T, Gelfuso GM, Rocha EM, Sarmento VH, de Freitas O, Lopez RF. A poloxamer/chitosan in situ forming gel with prolonged retention time for ocular delivery. Eur J Pharm Biopharm. 2010;75(2):186-93. doi: 10.1016/j.ejpb.2010.02.011.

5. Dartt DA. Regulation of mucin and fluid secretion by conjunctival epithelial cells. Prog Retin Eye Res. 2002;21(6):555-76.

6. Giese MJ, Speth RC. The ocular renin-angiotensin system: a therapeutic target for the treatment of ocular disease. Pharmacol Ther. 2014;142(1):11-32. doi: 10.1016/j.pharmthera.2013.11.002.

7. Presland A. Applied ocular physiology and anatomy. Anaesthesia \& Intensive Care Medicine. 2007;8(9):379-82. doi: 10.1016/j.mpaic.2007.07.001.

8. Huang HS, Schoenwald RD, Lach JL. Corneal penetration behavior of betablocking agents II: Assessment of barrier contributions. J Pharm Sci. 1983;72(11):1272-9.

9. Ang M, Baskaran M, Werkmeister RM, Chua J, Schmidl D, Aranha Dos Santos $\mathrm{V}$, et al. Anterior segment optical coherence tomography. Prog Retin Eye Res. 2018. doi: 10.1016/j.preteyeres.2018.04.002.

10. Prausnitz MR, Noonan JS. Permeability of cornea, sclera, and conjunctiva: a literature analysis for drug delivery to the eye. J Pharm Sci. 1998;87(12):1479-88.

11. Ramsay E, Del Amo EM, Toropainen E, Tengvall-Unadike U, Ranta VP, Urtti A, et al. Corneal and conjunctival drug permeability: Systematic comparison and pharmacokinetic impact in the eye. Eur J Pharm Sci. 2018;119:83-9. doi: 10.1016/j.ejps.2018.03.034.

12. Coleman AL. Glaucoma. Lancet. 1999;354(9192):1803-10. doi: 10.1016/S01406736(99)04240-3.

13. Semba K, Namekata K, Guo X, Harada C, Harada T, Mitamura Y. Reninangiotensin system regulates neurodegeneration in a mouse model of normal tension glaucoma. Cell Death Dis. 2014;5:e1333. doi: 10.1038/cddis.2014.296.

14. Quigley HA, Broman AT. The number of people with glaucoma worldwide in 2010 and 2020. Br J Ophthalmol. 2006;90(3):262-7. doi:

10.1136/bjo.2005.081224.

15. Rudnicka AR, Mt-Isa S, Owen CG, Cook DG, Ashby D. Variations in primary open-angle glaucoma prevalence by age, gender, and race: a Bayesian metaanalysis. Invest Ophthalmol Vis Sci. 2006;47(10):4254-61. doi: 10.1167/iovs.060299. 
16. Schwartz GF, Quigley HA. Adherence and persistence with glaucoma therapy.

Surv Ophthalmol. 2008;53 Suppl1(6):S57-68. doi:

10.1016/j.survophthal.2008.08.002.

17. Llobet A, Gasull X, Gual A. Understanding trabecular meshwork physiology: a key to the control of intraocular pressure? News Physiol Sci. 2003;18:205-9.

18. Tamm ER. The trabecular meshwork outflow pathways: structural and functional aspects. Exp Eye Res. 2009;88(4):648-55. doi: 10.1016/j.exer.2009.02.007.

19. Mozaffarieh M, Grieshaber MC, Flammer J. Oxygen and blood flow: players in the pathogenesis of glaucoma. Mol Vis. 2008;14:224-33.

20. Angelotti T, Hofmann F. Tissue-specific expression of splice variants of the mouse voltage-gated calcium channel alpha2/delta subunit. FEBS Lett. 1996;397(2-3):331-7.

21. Kriikku P, Wilhelm L, Rintatalo J, Hurme J, Kramer J, Ojanpera I. Pregabalin serum levels in apprehended drivers. Forensic Sci Int. 2014;243:112-6. doi: 10.1016/j.forsciint.2014.06.030.

22. Jeong KH, Woo HS, Kim CJ, Lee KH, Jeon JY, Lee SY, et al. Formulation of a modified-release pregabalin tablet using hot-melt coating with glyceryl behenate. Int J Pharm. 2015;495(1):1-8.

23. Cook J, Addicks W, Wu YH. Application of the biopharmaceutical classification system in clinical drug development--an industrial view. AAPS J. 2008;10(2):306-10. doi: 10.1208/s12248-008-9036-5.

24. Chintalapudi SR, Maria D, Di Wang X, Bailey JNC, consortium N, International Glaucoma Genetics c, et al. Systems genetics identifies a role for Cacna2d1 regulation in elevated intraocular pressure and glaucoma susceptibility. Nat Commun. 2017;8(1):1755. doi: 10.1038/s41467-017-00837-5.

25. Li N, Zhuang C, Wang M, Sun X, Nie S, Pan W. Liposome coated with lowmolecular weight chitosan and its potential use in ocular drug delivery. Int $\mathrm{J}$ Pharm. 2009;379:131-8.

26. Davis JL, Gilger BC, Robinson MR. Novel approaches to ocular drug delivery. Curr Opin Mol Ther. 2004;6(2):195-205.

27. Li CC, Abrahamson M, Kapoor Y, Chauhan A. Timolol transport from microemulsions trapped in HEMA gels. J Colloid Interface Sci. 2007;315(1):297306. doi: 10.1016/j.jcis.2007.06.054.

28. Lang J, C. Ocular drug delivery conventional ocular formulations. Adv Drug Deliv Rev. 1995;16(1):39-43.

29. Andres-Guerrero V, Alarma-Estrany P, Molina-Martinez IT, Peral A, HerreroVanrell R, Pintor J. Ophthalmic formulations of the intraocular hypotensive melatonin agent 5-MCA-NAT. Exp Eye Res. 2009;88(3):504-11. doi: 10.1016/j.exer.2008.11.004.

30. Llabot JM, Palma SD, Manzo RH, Allemandi DA. Design of novel antifungal mucoadhesive films. Part II. Formulation and in vitro biopharmaceutical evaluation. Int J Pharm. 2007;336(2):263-8. doi: 10.1016/j.ijpharm.2006.12.001.

31. Figueirêdo ES MA, Figueirêdo PF, Figueirêdo RS. Use of hyaluronic acid in Ophthalmology. Arq Bras Oftalmol. 2010;73(1):92-5. 
32. Kaur IP, Smitha R. Penetration enhancers and ocular bioadhesives: two new avenues for ophthalmic drug delivery. Drug Dev Ind Pharm. 2002;28(4):353-69. doi: 10.1081/DDC-120002997.

33. Rupenthal ID, Green CR, Alany RG. Comparison of ion-activated in situ gelling systems for ocular drug delivery. Part 1: physicochemical characterisation and in vitro release. Int J Pharm. 2011;411(1-2):69-77. doi: 10.1016/j.ijpharm.2011.03.042.

34. Bourges JL, Bloquel C, Thomas A, Froussart F, Bochot A, Azan F, et al. Intraocular implants for extended drug delivery: therapeutic applications. Adv Drug Deliv Rev. 2006;58(11):1182-202. doi: 10.1016/j.addr.2006.07.026.

35. Yasukawa T, Ogura Y, Kimura H, Sakurai E, Tabata Y. Drug delivery from ocular implants. Expert Opin Drug Deliv. 2006;3(2):261-73. doi: 10.1517/17425247.3.2.261.

36. Karlgard CC, Wong NS, Jones LW, Moresoli C. In vitro uptake and release studies of ocular pharmaceutical agents by silicon-containing and p-HEMA hydrogel contact lens materials. Int J Pharm. 2003;257(1-2):141-51.

37. Danion A, Brochu H, Martin Y, Vermette P. Fabrication and characterization of contact lenses bearing surface-immobilized layers of intact liposomes. J Biomed Mater Res A. 2007;82(1):41-51. doi: 10.1002/jbm.a.31147.

38. Short BG. Safety evaluation of ocular drug delivery formulations: techniques and practical considerations. Toxicol Pathol. 2008;36(1):49-62. doi: $10.1177 / 0192623307310955$.

39. Ghate D, Edelhauser HF. Ocular drug delivery. Expert Opin Drug Deliv. 2006;3(2):275-87. doi: 10.1517/17425247.3.2.275.

40. Morris B, Imrie F, Armbrecht AM, Dhillon B. Age-related macular degeneration and recent developments: new hope for old eyes? Postgrad Med J. 2007;83(979):301-7. doi: 10.1136/pgmj.2006.052944.

41. Mainardes RM, Urban MC, Cinto PO, Khalil NM, Chaud MV, Evangelista RC, et al. Colloidal carriers for ophthalmic drug delivery. Curr Drug Targets. 2005;6(3):363-71.

42. Changez M, Varshney M. Aerosol-OT Microemulsions as Transdermal Carriers of Tetracaine Hydrochloride. Drug Dev Ind Pharm 2000;26(5):507-12.

43. Heuschkel S, Goebel A, Neubert RH. Microemulsions--modern colloidal carrier for dermal and transdermal drug delivery. J Pharm Sci. 2008;97(2):603-31. doi: $10.1002 /$ jps.20995.

44. Sharma SC, Tsuchiya K, Sakai K, Sakai H, Abe M, Komura S, et al. Formation and characterization of microemulsions containing polymeric silicone. Langmuir. 2008;24(15):7658-62. doi: 10.1021/la801205q.

45. Callender SP, Mathews JA, Kobernyk K, Wettig SD. Microemulsion utility in pharmaceuticals: Implications for multi-drug delivery. Int J Pharm. 2017;526(12):425-42. doi: 10.1016/j.ijpharm.2017.05.005.

46. Lawrence MJ. Surfactant Systems - Microemulsions and Vesicles as Vehicles for Drug-Delivery. European Journal of Drug Metabolism and Pharmacokinetics. 1994;19(3):257-69. doi: Doi 10.1007/Bf03188929. 
47. Moreno MA, Ballesteros MP, Frutos P. Lecithin-based oil-in-water microemulsions for parenteral use: Pseudoternary phase diagrams, characterization and toxicity studies. Journal of Pharmaceutical Sciences. 2003;92(7):1428-37. doi: 10.1002/jps.10412.

48. Santos P, Watkinson AC, Hadgraft J, Lane ME. Application of microemulsions in dermal and transdermal drug delivery. Skin Pharmacol Physiol. 2008;21(5):24659. doi: 10.1159/000140228.

49. Gupta S, Moulik SP. Biocompatible microemulsions and their prospective uses in drug delivery. J Pharm Sci. 2008;97(1):22-45. doi: 10.1002/jps.21177.

50. Kumar R, Sinha VR. Preparation and optimization of voriconazole microemulsion for ocular delivery. Colloids Surf B Biointerfaces. 2014;117:82-8. doi: 10.1016/j.colsurfb.2014.02.007.

51. Guo RX, Fu X, Chen J, Zhou L, Chen G. Preparation and Characterization of Microemulsions of Myricetin for Improving Its Antiproliferative and Antioxidative Activities and Oral Bioavailability. J Agric Food Chem. 2016;64(32):6286-94. doi: 10.1021/acs.jafc.6b02184.

52. Silva AE, Barratt G, Cheron M, Egito ES. Development of oil-in-water microemulsions for the oral delivery of amphotericin B. Int J Pharm. 2013;454(2):641-8. doi: 10.1016/j.ijpharm.2013.05.044.

53. Schulman JH, Stoeckenius W, Prince LM. Mechanism of Formation and Structure of Micro Emulsions by Electron Microscopy. J Phys Chem. 1959;63(10):167780 .

54. McClements DJ. Nanoemulsions versus microemulsions: terminology, differences, and similarities. Soft Matter. 2012;8(6):1719-29. doi: 10.1039/c2sm06903b.

55. Attwood D, Florence AT. surfactants. Physical Pharmacy Chapter 4: vol 2nd Edition Pharmaceutical Press, UK. 2012:pp. 43.

56. Tadros TF. Physical chemistry of surfactant solutions. Applied Surfactants: Principles and Applications. Chapter 2: Wiley-VCH, UK,. 2005: pp. 19.

57. Myers D. The organic chemistry of surfactants. Surfactant Science and Technology. Chapter 2: Wiley-VCH, UK, vol 3rd edition Wiley Interscience, USA. 2005:pp. 29.

58. Winsor PA. Hydrotropy, solubilisation and related emulsification processes. Trans Faraday Soc. 1948;44:376-98.

59. Nordiyana MSW, Khalil M, Jan BM, Ali BS, Tong CW. Formation and Phase Behavior of Winsor Type III Jatropha curcas-Based Microemulsion Systems. Journal of Surfactants and Detergents. 2016;19(4):701-12. doi: 10.1007/s11743016-1814-y.

60. Griffin WC. Calculation of HLB values of non-ionic surfactants, Journal of Society of Cosmetic Chemists. Journal of Society of Cosmetic Chemists 1954;5:249-56.

61. Rosen MJ, Kunjappu JT. Emulsification by surfactants, Surfactants and Interfacial Phenomena. Chapter 8: 4th edition John Wiley \& Sons Publisher, New Jersey, USA,. 2012:pp. 336.

62. Vandamme TF. Microemulsions as ocular drug delivery systems: recent developments and future challenges. Prog Retin Eye Res. 2002;21(1):15-34. 
63. Moulik SP, Paul BK. Structure, dynamics and transport properties of microemulsions. Advances in Colloid and Interface Science. 1998;78(2):99 195.

64. Mason TG, Wilking JN, Meleson K, Chang CB, Graves SM. Nanoemulsions: formation, structure, and physical properties. J Phys-Condens Mat. 2006;18(41):R635-R66. doi: 10.1088/0953-8984/18/41/R01.

65. Peng CC, Bengani LC, Jung HJ, Leclerc J, Gupta C, Chauhan A. Emulsions and microemulsions for ocular drug delivery. Journal of Drug Delivery Science and Technology. 2011;21(1):111-21. doi: 10.1016/s1773-2247(11)50010-3.

66. Lin C-C, Lin H-Y, Chen H-C, Yu M-W, Lee M-H. Stability and characterisation of phospholipid-based curcumin-encapsulated microemulsions. Food Chemistry. 2009;116(4):923-8. doi: 10.1016/j.foodchem.2009.03.052.

67. Vasiljevic D, Parojcic J, Primorac M, Vuleta G. An investigation into the characteristics and drug release properties of multiple $\mathrm{W} / \mathrm{O} / \mathrm{W}$ emulsion systems containing low concentration of lipophilic polymeric emulsifier. Int J Pharm. 2006;309(1-2):171-7. doi: 10.1016/j.ijpharm.2005.11.034.

68. Lv FF, Zheng LQ, Tung CH. Phase behavior of the microemulsions and the stability of the chloramphenicol in the microemulsion-based ocular drug delivery system. Int J Pharm. 2005;301(1-2):237-46. doi: 10.1016/j.ijpharm.2005.06.006.

69. Dahan A, Hoffman A. Enhanced gastrointestinal absorption of lipophilic drugs. In: Touitou, E., Barry, B. (Eds.), Enhancement in Drug Delivery. Chapter 6: CRC Press, USA. 2011:pp. 111.

70. Gallarate M, Chirio D, Bussano R, Peira E, Battaglia L, Baratta F, et al. Development of $\mathrm{O} / \mathrm{W}$ nanoemulsions for ophthalmic administration of timolol. Int J Pharm. 2013;440(2):126-34. doi: 10.1016/j.ijpharm.2012.10.015.

71. Gujral RS, Haque SM, Kumar S. A novel method for the determination of pregabalin in bulk pharmaceutical formulations and human urine samples. African Journal of Pharmacy and Pharmacology. 2009;3(6):327-34.

72. Savic V, Todosijevic M, Ilic T, Lukic M, Mitsou E, Papadimitriou V, et al. Tacrolimus loaded biocompatible lecithin-based microemulsions with improved skin penetration: Structure characterization and in vitro/in vivo performances. Int J Pharm. 2017;529(1-2):491-505. doi: 10.1016/j.jpharm.2017.07.036.

73. Zhang J, Michniak-Kohn B. Investigation of microemulsion microstructures and their relationship to transdermal permeation of model drugs: ketoprofen, lidocaine, and caffeine. Int J Pharm. 2011;421(1):34-44. doi: 10.1016/j.jpharm.2011.09.014.

74. Fialho SL, da Silva-Cunha A. New vehicle based on a microemulsion for topical ocular administration of dexamethasone. Clin Exp Ophthalmol. 2004;32(6):62632. doi: $10.1111 /$ j.1442-9071.2004.00914.x.

75. Chen H, Mou D, Du D, Chang X, Zhu D, Liu J, et al. Hydrogel-thickened microemulsion for topical administration of drug molecule at an extremely low concentration. Int J Pharm. 2007;341(1-2):78-84. doi: 10.1016/j.ijpharm.2007.03.052.

76. Lawrence MJ, Rees GD. Microemulsion-based media as novel drug delivery systems. Adv Drug Deliv Rev. 2012;64:175-93. 
77. Kaukonen AM, Boyd BJ, Porter CJ, Charman WN. Drug solubilization behavior during in vitro digestion of simple triglyceride lipid solution formulations. Pharm Res. 2004;21(2):245-53.

78. Ren Q, Deng C, Meng L, Chen Y, Chen L, Sha X, et al. In vitro, ex vivo, and in vivo evaluation of the effect of saturated fat acid chain length on the transdermal behavior of ibuprofen-loaded microemulsions. J Pharm Sci. 2014;103(6):1680-91. doi: $10.1002 /$ jps.23958.

79. Ammar HO, Salama HA, Ghorab M, Mahmoud AA. Nanoemulsion as a potential ophthalmic delivery system for dorzolamide hydrochloride. AAPS PharmSciTech. 2009;10(3):808-19. doi: 10.1208/s12249-009-9268-4.

80. BASF Fine Chemicals. Cremophor ${ }^{\circledR}$ EL. Technical leaflet, ME 074:5, Ludwigshafen, Germany: BASF Fine Chemicals, 1997.

81. Bietti GB, Virno M, Pecori-Giraldi J. Propylenglycol. A new osmotic agent for ophthalmic uses. Doc Ophthalmol. 1973;34(1):77-92.

82. Grant WM, Schuman JS. Toxicology of the eye. 4th ed: Spring-field; 1993.

83. Liu Z, Zhang X, Li J, Liu R, Shu L, Jin J. Effects of Labrasol on the corneal drug delivery of baicalin. Drug Deliv. 2009;16(7):399-404. doi: 10.1080/10717540903126165.

84. Benelli U. Systane lubricant eye drops in the management of ocular dryness. Clin Ophthalmol. 2011;5:783-90. doi: 10.2147/OPTH.S13773.

85. Alany RG, Rades T, Nicoll J, Tucker IG, Davies NM. W/O microemulsions for ocular delivery: evaluation of ocular irritation and precorneal retention. J Control Release. 2006;111(1-2):145-52. doi: 10.1016/j.jconrel.2005.11.020.

86. Kalam MA, Alshamsan A, Aljuffali IA, Mishra AK, Sultana Y. Delivery of gatifloxacin using microemulsion as vehicle: formulation, evaluation, transcorneal permeation and aqueous humor drug determination. Drug Deliv. 2016;23(3):896907.

87. Ibrahim MM, Abd-Elgawad AH, Soliman OA, Jablonski MM. Natural Bioadhesive Biodegradable Nanoparticle-Based Topical Ophthalmic Formulations for Management of Glaucoma. Transl Vis Sci Technol. 2015;4(3):12. doi: 10.1167/tvst.4.3.12.

88. Ibrahim MM, Abd-Elgawad AH, Soliman OA, Jablonski MM. Stability and Ocular Pharmacokinetics of Celecoxib-Loaded Nanoparticles Topical Ophthalmic Formulations. J Pharm Sci. 2016;105(12):3691-701. doi: 10.1016/j.xphs.2016.09.019.

89. Maria DN, Abd-Elgawad AH, Soliman OA, El-Dahan MS, Jablonski MM. Nimodipine Ophthalmic Formulations for Management of Glaucoma. Pharm Res. 2017;34(4):809-24.

90. Mayol L, Quaglia F, Borzacchiello A, Ambrosio L, La Rotonda MI. A novel poloxamers/hyaluronic acid in situ forming hydrogel for drug delivery: rheological, mucoadhesive and in vitro release properties. Eur J Pharm Biopharm. 2008;70(1):199-206. doi: 10.1016/j.ejpb.2008.04.025. 
91. Tayel SA, El-Nabarawi MA, Tadros MI, Abd-Elsalam WH. Promising ionsensitive in situ ocular nanoemulsion gels of terbinafine hydrochloride: design, in vitro characterization and in vivo estimation of the ocular irritation and drug pharmacokinetics in the aqueous humor of rabbits. Int J Pharm. 2013;443(12):293-305. doi: 10.1016/j.ijpharm.2012.12.049.

92. Ibrahim MM, Abd-Elgawad AE, Soliman OA, Jablonski MM. Nanoparticle-based topical ophthalmic formulations for sustained celecoxib release. J Pharm Sci. 2013;102(3):1036-53. doi: 10.1002/jps.23417.

93. Balguri SP, Adelli GR, Janga KY, Bhagav P, Majumdar S. Ocular disposition of ciprofloxacin from topical, PEGylated nanostructured lipid carriers: Effect of molecular weight and density of poly (ethylene) glycol. Int J Pharm. 2017;529(12):32-43. doi: 10.1016/j.ijpharm.2017.06.042.

94. Battistini FD, Tartara LI, Boiero C, Guzman ML, Luciani-Giaccobbe LC, Palma $\mathrm{SD}$, et al. The role of hyaluronan as a drug carrier to enhance the bioavailability of extended release ophthalmic formulations. Hyaluronan-timolol ionic complexes as a model case. Eur J Pharm Sci. 2017;105:188-94. doi: 10.1016/j.ejps.2017.05.020.

95. Hegde RR, Bhattacharya SS, Verma A, Ghosh A. Physicochemical and pharmacological investigation of water/oil microemulsion of non-selective beta blocker for treatment of glaucoma. Curr Eye Res. 2014;39(2):155-63. doi: 10.3109/02713683.2013.833630.

96. Nookala S, Gandrakota R, Wohabrebbi A, Wang X, Howell D, Giorgianni F, et al. In search of the identity of the XAP-1 antigen: a protein localized to cone outer segments. Invest Ophthalmol Vis Sci. 2010;51(5):2736-43. doi: 10.1167/iovs.094286.

97. Chintalapudi SR, Maria DN, Wang X, Surbhi S, Wiggs JL, Williams RW, et al. Cacna2d1: a novel therapeutic target for lowering IOP. Poster presented at 2016 ARVO meeting.

98. Seijo B, E.Fattal, L.Roblot-Treupel, P.Couvreur. Design of nanoparticles of less than $50 \mathrm{~nm}$ diameter: preparation, characterization and drug loading. Int J Pharm. 1990;62(1):1-7. doi: 10.1016/0378-5173(90)90024-X.

99. Scholes PD, Coombes AGA, Illum L, Daviz SS, Vert M, Davies MC. The preparation of sub-200 $\mathrm{nm}$ poly(lactide-co-glycolide) microspheres for sitespecific drug delivery. J Control Release. 1993;25(1-2):145-53. doi: 10.1016/0168-3659(93)90103-C.

100. Tenjaria S. Crit Rev Ther Drug Carrier Syst. 1999;16(5):461-521.

101. Nidhin M, Indumathy R, Sreeram KJ, Nair BU. Synthesis of iron oxide nanoparticles of narrow size distribution on polysaccharide templates. Bulletin of Materials Science. 2008;31(1):93-6. doi: DOI 10.1007/s12034-008-0016-2.

102. Mali KK, Dhawale SC, Dias RJ. Microemulsion Based Bioadhesive Gel of Itraconazole Using Tamarind Gum: In-vitro and Ex-vivo Evaluation. Marmara Pharm J. 2017;21(3):688-700. doi: 10.12991/marupj.323593.

103. Naga Sravan Kumar Varma V, Maheshwari PV, Navya M, Reddy SC, Shivakumar HG, Gowda DV. Calcipotriol delivery into the skin as emulgel for effective permeation. Saudi Pharm J. 2014;22(6):591-9. doi:

10.1016/j.jsps.2014.02.007. 
104. Sogias IA, Williams AC, Khutoryanskiy VV. Why is chitosan mucoadhesive?

Biomacromolecules. 2008;9(7):1837-42. doi: 10.1021/bm800276d.

105. Novoa-Carballal R, Fernandez-Megia E, Riguera R. Dynamics of chitosan by (1)h NMR relaxation. Biomacromolecules. 2010;11(8):2079-86. doi:

10.1021/bm100447f.

106. Menchicchi B, Fuenzalida JP, Bobbili KB, Hensel A, Swamy MJ, Goycoolea FM. Structure of chitosan determines its interactions with mucin. Biomacromolecules. 2014;15(10):3550-8. doi: 10.1021/bm5007954.

107. Modi S, Anderson BD. Determination of drug release kinetics from nanoparticles: overcoming pitfalls of the dynamic dialysis method. Mol Pharm. 2013;10(8):3076-89. doi: 10.1021/mp400154a.

108. Araie M, Shirasawa E, Hikita M. Effect of oxidized glutathione on the barrier function of the corneal endothelium. Invest Ophthalmol Vis Sci. 1988;29(12):1884-7.

109. Araie M, Shirasawa E, Ohashi T. Intraocular irrigating solutions and permeability of the blood-aqueous barrier. Arch Ophthalmol. 1990;108(6):882-5.

110. Quinteros DA, Tártara LI, Palma SD, Manzo RH, Allemandi DA. Ocular Delivery of Flurbiprofen Based on Eudragi(®)R E-Flurbiprofen Complex Dispersed in Aqueous Solution: Preparation, Characterization, In Vitro Corneal Penetration, and Ocular Irritation. J Pharm Sci. 2014;103(12):3859-68.

111. Jessen BA, Shiue MH, Kaur H, Miller P, Leedle R, Guo H, et al. Safety assessment of subconjunctivally implanted devices containing latanoprost in Dutch-belted rabbits. J Ocul Pharmacol Ther. 2013;29(6):574-85. doi: 10.1089/jop.2012.0190.

112. Maria DN, Abd-Elgawad AH, Soliman OA, El-Dahan MS, Jablonski MM. Nimodipine Ophthalmic Formulations for Management of Glaucoma. Pharmaceutical Research. 2017;34(4):809-24.

113. Xu M, McCanna DJ, Sivak JG. Use of the viability reagent PrestoBlue in comparison with alamarBlue and MTT to assess the viability of human corneal epithelial cells. J Pharmacol Toxicol Methods. 2015;71:1-7. doi: 10.1016/j.vascn.2014.11.003.

114. Janga KY, Tatke A, Balguri SP, Lamichanne SP, Ibrahim MM, Maria DN, et al. Ion-sensitive in situ hydrogels of natamycin bilosomes for enhanced and prolonged ocular pharmacotherapy: in vitro permeability, cytotoxicity and in vivo evaluation. Artif Cells Nanomed Biotechnol. 2018:1-12. doi:

10.1080/21691401.2018.1443117.

115. Liu Y, Peterson DA, Kimura H, Schubert D. Mechanism of cellular 3-(4,5dimethylthiazol-2-yl)-2,5-diphenyltetrazolium bromide (MTT) reduction. J Neurochem. 1997;69(2):581-93.

116. Andres-Guerrero V, Vicario-de-la-Torre M, Molina-Martinez IT, Benitez-delCastillo JM, Garcia-Feijoo J, Herrero-Vanrell R. Comparison of the in vitro tolerance and in vivo efficacy of traditional timolol maleate eye drops versus new formulations with bioadhesive polymers. Invest Ophthalmol Vis Sci. 2011;52(6):3548-56. doi: 10.1167/iovs.10-6338.

117. Kumar S, Himmelstein KJ. Modification of in situ gelling behavior of carbopol solutions by hydroxypropyl methylcellulose. J Pharm Sci. 1995;84(3):344-8. 
118. Naveh N, Muchtar S, Benita S. Pilocarpine Incorporated into a Submicron Emulsion Vehicle Causes an Unexpectedly Prolonged Ocular Hypotensive Effect in Rabbits. Journal of Ocular Pharmacology and Therapeutics. 1994;10(3).

119. Guidance for Industry Q1A(R2) Stability Testing of New Drug Substances and Products. In: Administration USDoHaHSFaD, (CBER) CfDEaRCCfBEaR, editors.2003.

120. Dehghani F, Farhadian N, Golmohammadzadeh S, Biriaee A, Ebrahimi M, Karimi M. Preparation, characterization and in-vivo evaluation of microemulsions containing tamoxifen citrate anti-cancer drug. European Journal of Pharmaceutical Sciences. 2017;96:479-89. doi: 10.1016/j.ejps.2016.09.033.

121. Wan T, Xu T, Pan J, Qin M, Pan W, Zhang G, et al. Microemulsion based gel for topical dermal delivery of pseudolaric acid B: In vitro and in vivo evaluation. Int J Pharm. 2015;493(1-2):111-20. doi: 10.1016/j.ijpharm.2015.07.058.

122. Zhu W, Guo C, Yu A, Gao Y, Cao F, Zhai G. Microemulsion-based hydrogel formulation of penciclovir for topical delivery. Int J Pharm. 2009;378(1-2):152-8. doi: 10.1016/j.ijpharm.2009.05.019.

123. Loftsson T. Drug stabiity for pharmaceutical scientists: Academic Press; 2013.

124. Rakesh Kumar R, Sinha VR. Preparation and optimization of voriconazole microemulsion forocular delivery. Colloids and Surfaces B: Biointerfaces. 2014;117:82-8.

125. Alam MA, Al-Janoobi FI, Alzahrani KA, Al-Agamy MH, Abdelgalil AA, AlMohizea AM. In-vitro efficacies of topical microemulsions of clotrimazole and ketoconazole; and in-vivo performance of clotrimazole microemulsion. Journal of Drug Delivery Science and Technology. 2017;39:408-16. doi: 10.1016/jjddst.2017.04.025.

126. Ustundag-Okur N, Gokce EH, Egrilmez S, Ozer O, Ertan G. Novel ofloxacinloaded microemulsion formulations for ocular delivery. J Ocul Pharmacol Ther. 2014;30(4):319-32. doi: 10.1089/jop.2013.0114. 


\section{VITA}

Doaa Nabih Ahmed Maria was born in Damietta, Egypt in 1984, the daughter of Nabih Ahmed Maria and H. M. O. After completing her bachelor's degree as Excellent with honor and also with $5^{\text {th }}$ achiever graduation ranking at Faculty of Pharmacy, Mansoura university, Egypt in November 2005, she got a position as a demonstrator in the same college in the department of pharmaceutics and began her research work in the field of drug targeting and topical ocular drug delivery. In April, 2011, Doaa got a Master's of Pharmaceutical Science degree (Thesis). In May, 2011 she began to work as an assistant lecturer in the same department, Mansoura University, Egypt. In March, 2013 she joined the Ph.D. program in the same college then in July, 2013 she got $A$ Scholarship Award from Egyptian Joint Supervision Program. In December, 2013, Doaa joined Dr. Monica M. Jablonski lab to continue her Ph. D research work, Department of Ophthalmology, University of Tennessee Health Science Center (UTHSC). In Fall 2015, She joined UTHSC as a doctoral student in Pharmaceutical Science, Drug Delivery Track. During her time at UTHSC, Doaa has made several presentations in the department of Pharmaceutical Science. Also, in April 2017 and 2018 she presented posters in Graduation Research Day at UTHSC. During her doctoral studies, she published four papers in peer reviewed journals. After having successfully defended her dissertation in October 2018, Doaa will be awarded her doctoral degree in December 2018. 